\title{
34. DEEP SEA DRILLING PROJECT LEG 58 ABYSSAL BASALTS FROM THE SHIKOKU BASIN: THEIR PETROLOGY AND MAJOR-ELEMENT GEOCHEMISTRY
}

\author{
Henry J. B. Dick ${ }^{1}$, Nicholas G. Marsh ${ }^{2}$, and Thomas D. Bullen ${ }^{1}$
}

\begin{abstract}
The Shikoku Basin is floored at the three sites drilled during DSDP Leg 58 by sill complexes representing widespread "off-axis" extensional volcanism. Similar sill complexes appear to have been drilled during DSDP Leg 15 in the Caribbean. At two of the three drill sites, the sill complexes were penetrated, and are underlain by intercalated pillow basalts, shallow intrusives, and sheet flows. One sill of differentiated alkali olivine basalt was found in the sill complex at Site 444. All the basalts, except the one alkaline sill, appear to be for the most part typical of mid-ocean-ridge tholeiites. Unusual features include very high basalt vesicularities - and therefore primary volatile contents - and a somewhat transitional character in the $\mathrm{Al}_{2} \mathrm{O}_{3}-\mathrm{FeO}-\mathrm{MgO}$ discriminant diagram at some sites. The Leg 58 results help define criteria for the identification of back-arc-basin ophiolites, including: a stratigraphic section with intercalated sills, sediments, and silicic ash; highly vesiculated basalts; some basalts transitional between MORB and continental and island-arc basalt suites; and an unusual abundance of alkali basalts.
\end{abstract}

\section{INTRODUCTION}

The Shikoku Basin is an inter-arc basin south of the Island of Shikoku, between the inactive Kyushu-Palau Ridge to the west and the Iwo Jima arc to the east. Immediately east of the Iwo Jima Ridge is the presently active Bonin arc, and an intervening trough which may represent a new inter-arc basin forming east of the Shikoku Basin. It is believed that the Kyushu-Palau ridge became inactive about $28 \mathrm{Ma}$, while the oldest rocks dated in the Bonin islands are 40 m.y. old (Kobayashi and Isezaki, 1976). The Shikoku Basin has high heat flow (Uyeda, 1976), a central band of thin sediment (Murauchi and Asanuma, 1974), axially lineated topography and magnetic anomalies (Watts and Weissel, 1975; Kobayashi and Isezaki, 1976), and a seismic signature typical of ocean crust (e.g., Murauchi et al., 1968). In view of this, the Shikoku Basin is believed to have formed as a result of rifting of an old island arc and the formation of a new basin floored by oceanic crust between the inactive arc remanent to the west and a stillactive part to the east (Karig, 1970; Watts and Weissel, 1975; Kobayashi and Isezaki, 1976). Given the present lack of seismic activity in the basin and the inactive arcs to the east and west, it is believed that the basin is also presently inactive (Kobayashi and Isezaki, 1976).

Lying to the east of a major island arc, which itself is far from the Asian landmass, the Shikoku Basin pro-

\footnotetext{
${ }^{1}$ Woods Hole Oceanographic Institution, Woods Hole, Massachusetts.

${ }^{2}$ University of Birmingham, Birmingham, England.
}

vides an excellent opportunity to study the petrogenesis and development of a back-arc basin without the complication of the possible involvement of a continental margin.

\section{VOLCANIC STRATIGRAPHY}

During Leg 58, basaltic basement was drilled at three sites in the Shikoku Basin (Figure 1): 27.6 meters in Hole 442A, 160.8 meters nearby in Hole 442B, 122 meters in Hole 443, and 44.5 meters in Hole 444A. Site 442 is on magnetic anomaly $6(19-20 \mathrm{~m}$.y.), west of the Shikoku Basin paleo-spreading center postulated by Kobayashi and Isezaki (1976), while Sites 443 and 444 are along strike to the east of the spreading center, on anomaly 6 A (19-21 m.y.). Immediately overlying and in some cases interbedded with the basalt are hemipelagic clays with numerous ash layers. In all, some 354 meters of basalt were drilled in which 39 massive basalt sills and flows were identified, and a minimum of seven separate pillow-lava units. The sills and flows range from less than 1 meter to over 34 meters thick, averaging 5.8 meters. The pillow lavas range from a 57.5 -meter-thick sub-unit at Site 442 to an approximately 2 -meter-thick sub-unit at Site 443.

\section{Site 442}

Stratigraphy at Site 442,59 meters of massive, aphyric, vesicular basalt (unit 1, 16 sub-units) is underlain by $102 \mathrm{~m}$ of aphyric, vesicular pillow basalt with two intercalated massive basalt sub-units (units 2 and 3 ). Units 1 and 2 are separated by $2 \mathrm{~m}$ of sediment. Nannofossil chalk and limestone are locally present in small quantities in the pillow basalts (Plate 1). 


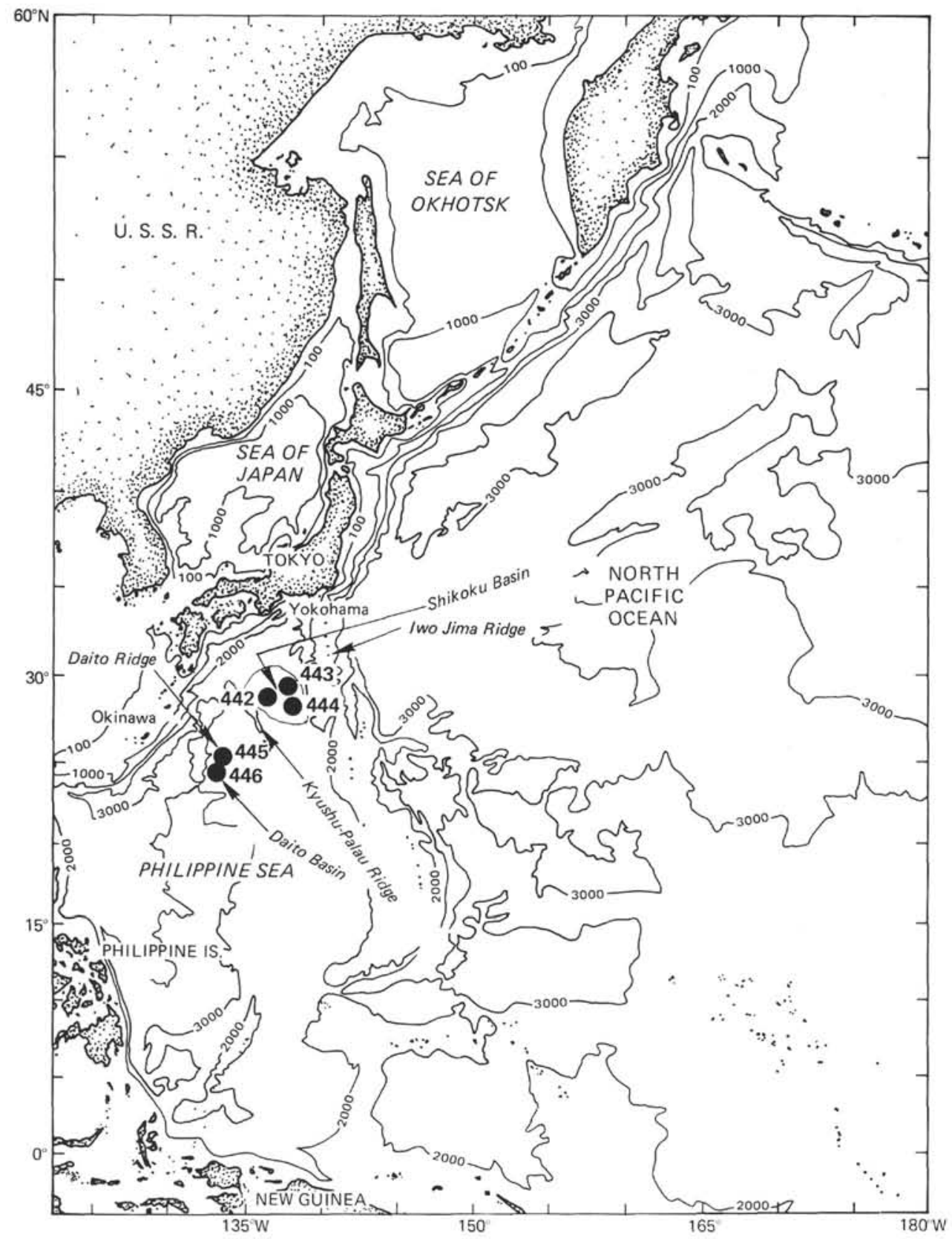

Figure 1. Map of the Shikoku Basin.

The identification of the sediment layer between units 1 and 2 is based on (1) anomalously high drilling rates for a 2-meter interval; (2) the presence of mud in the core catcher from this interval containing fossils older than those in the oldest sediment overlying unit 1 (15-17 m.y. vs. 18-21 m.y.; see Site 442 report, this volume); and (3) the location of this interval at the major lithologic break in the basalt sequence.

The sub-units in the upper 30 meters of basement at Holes $442 \mathrm{~A}$ and $442 \mathrm{~B}$ correlate well. If the very minor sub-unit $1 \mathrm{~F}$ is ignored, there are the same number of sub-units-with similar petrography, vesicularity, and alteration-in the upper 30 meters of the basement in each hole. This suggests that the massive basalts are la- terally continuous and therefore not dikes. Unlike the pillow basalts, the chilled margins of the massive basalts tend to be either fine-grained or cryptocrystalline, glassy basalt, rather than glass, as are those in sheeted dike complexes. The two intercalated massive basalt units ( 5.5 and $8.5 \mathrm{~m}$ thick) in the pillow basalts could be either massive portions of pillow lava flows or sills.

\section{Petrography}

All the basalts are aphyric ( $<1 \%$ phenocrysts). Modal analyses are given in Table 1 . Of the 94 thin sections examined, only 21 contained a few phenocrysts (11 with plagioclase only, six with plagioclase and olivine, three with plagioclase and pyroxene, and one with a single 
TABLE 1

Modal Analyses of Representative Hole 442B Basalts and Diabases

\begin{tabular}{|c|c|c|c|c|c|c|c|c|c|c|c|}
\hline \multirow[b]{2}{*}{ Interval } & \multirow[b]{2}{*}{ Matrix } & \multirow[b]{2}{*}{ Vesicles } & \multirow[b]{2}{*}{ Glass } & \multicolumn{3}{|c|}{ Groundmass or Microphenocrysts } & \multicolumn{3}{|c|}{ Phenocrysts } & \multirow{2}{*}{$\begin{array}{l}\text { Titano- } \\
\text { magnetite }\end{array}$} & \multirow[b]{2}{*}{ Points Counted } \\
\hline & & & & Plagioclase & Clinopyroxene & Olivine & Plagioclase & Clinopyroxene & Olivine & & \\
\hline 42 & 63.2 & 36.8 & - & - & - & - & - & - & - & - & - \\
\hline $5-4$ & 72.7 & 26.3 & $\mathrm{nc}^{\mathrm{a}}$ & $\mathrm{nc}$ & $n c$ & $\mathrm{nc}$ & $\mathrm{nc}$ & $\mathrm{nc}$ & nc & $\mathrm{nc}$ & 1531 \\
\hline $8-1,32-34$ & 44.3 & 0.6 & - & 38.4 & 16.7 & - & - & - & - & - & 1236 \\
\hline $9-1,103-105$ & 24.5 & 15.6 & - & 29.1 & 22.9 & $6.3^{b}$ & - & - & - & 1.7 & - \\
\hline $10-1,111-113$ & \multicolumn{6}{|c|}{$\sim 20$ (too fine grained, aphyric) } & \multicolumn{5}{|l|}{ - } \\
\hline \\
\hline $\begin{array}{l}\text { glass zone } \\
\text { cryptocrystalline }\end{array}$ & $\begin{array}{l}28.0 \\
84.0\end{array}$ & $\begin{array}{r}0.2 \\
15.0\end{array}$ & $\begin{array}{c}50.9 \\
-\end{array}$ & $\begin{array}{l}1.3 \\
1.0\end{array}$ & - & $\begin{array}{c}19.6 \\
-\end{array}$ & - & - & $\begin{array}{l}- \\
-\end{array}$ & $\begin{array}{l}\mathrm{ne} \\
\mathrm{ne}\end{array}$ & $\begin{array}{l}929 \\
996\end{array}$ \\
\hline 142 & 74.0 & 26.0 & nc & $\mathrm{nc}$ & $\mathrm{nc}$ & nc & $\mathrm{nc}$ & $\mathrm{nc}$ & $n c$ & $\mathrm{nc}$ & 1060 \\
\hline $15-1$ & 75.0 & 25.0 & $\mathrm{nc}$ & $\mathrm{nc}$ & $\mathrm{nc}$ & $\mathrm{nc}$ & $\mathrm{nc}$ & $\mathrm{nc}$ & $\mathrm{nc}$ & $\mathrm{nc}$ & 944 \\
\hline $16-1$ & 74 & 26 & ne & $\mathrm{nc}$ & nc & $\mathrm{nc}$ & $\mathrm{nc}$ & $\mathrm{nc}$ & $n c$ & $\mathrm{ne}$ & 978 \\
\hline $17-1,12-60$ & 89.9 & 7.5 & - & 1.5 & 0.9 & 0.1 & - & - & - & $\mathrm{nc}$ & 1173 \\
\hline $18-1,7-8$ & 84.1 & 11.5 & - & 1.4 & 2.8 & 0.1 & - & - & - & $\mathrm{nt}$ & 1335 \\
\hline $19-2,65-70$ & 82.2 & 8.2 & - & 7.8 & 1.7 & 0.1 & - & - & - & $\mathrm{nc}$ & \\
\hline
\end{tabular}

a "nc" - not counted.

${ }^{\mathrm{b}}$ Calcite pseudomorphs after olivine.

pyroxene phenocryst). All the phenocrysts are small (plagioclase, 0.5-2.0 mm; olivine, 0.5-1.9 mm; pyroxene, $0.6-1.0 \mathrm{~mm})$.

The Site 442 basalts and diabases appear mineralogically typical of mid-ocean-ridge basalt (MORB). Plagioclase is the most abundant phase, followed by clinopyroxene and minor titanomagnetite. Olivine and olivine pseudomorphs are present in the interior of all the massive cooling units except $1 \mathrm{D}$ and $1 \mathrm{~L}$, and in small amounts in 16 of 19 glassy chill zones, examined in thin section, from the pillow lavas and sills. Olivine appears to be entirely absent in the relatively crystalline interiors of the pillows, even a few centimeters from where it is present in the chill zone. Titanomagnetite is present in variable amounts in all the non-glassy thin sections. Spinel occurs as small rust-red octahedra, generally enclosed in olivine, plagioclase, or, less commonly, in pyroxene microphenocrysts.

\section{Massive Basalts}

Many of the Site 442 massive sub-units are diabases (1D, E, K, M, N, and P). These contain 65 to 95 per cent medium-grained plagioclase, pyroxene, and in some cases olivine, in a fine-grained groundmass of intersertal to intergranular plagioclase, pyroxene, microlites, oxides, clay, and calcite. In a few cases, the alteration products may replace glass. Pyroxene in these sills ranges from clear to pale brown and sub-ophitic to poikilitic about fine- to medium-grained plagioclase and olivine. The proportion of olivine appears to vary with depth in the thickest unit $(1 \mathrm{~K})$, suggesting that some olivine settling occurred during crystallization. As in the basalts, vesicularities may be very high: Sample 4-2, $110-115 \mathrm{~cm}$ consists of 90 per cent medium-grained intergrown plagioclase and pyroxene laths with about 5 per cent intersertal palagonite and 37 per cent vesicles, producing a diktytaxitic diabase with intersertal texture.

The bimodal crystal-size distribution of many of the massive basalts was also found in many of the shallow intrusives (with baked contacts) at Site 446 in the Daito Basin, as well as at the other Shikoku Basin sites. Despite the porphyritic ("luster mottled") appearance of many of these diabases, their chill zones contain only a few small phenocrysts, indicating little intratelluric crystallization prior to emplacement. We speculate, then, that this bimodality of grain size is a function of the cooling of a shallow intrusive in wet sediments.

The finer-grained, massive cooling units (basalts) are similar in most respects to the diabases. Calcite, rather than clay, is the principal pseudomorph after olivine, and pyroxene tends to be subophitic rather than ophitic or porphyritic. A glassy chill zone from sub-unit $1 \mathrm{~A}$ contains numerous plagioclase and pyroxene microphenocrysts, while a second from sub-unit 1I contains only abundant calcite pseudomorphs after olivine, suggesting some variability of the initial liquidus phases of these units.

While the textures of the massive basalts are unusual for abyssal basalts, they are typical of subaerial lava flows and shallow intrusives. Although a thick flow may have a diabasic texture and a thin sill a glassy one, we feel that some, if not all, of the massive unit 1 basalts are shallow sills, largely because of their lack of glassy chilled margins and the absence of intercalated pillow basalts.

\section{Pillow Basalts}

Textural gradations from interior to glassy margins of the pillow basalts at Site 442 are typical of mid-oceanridge pillow basalts. In the chill zones, olivine microphenocrysts are present in trace amounts, while pyroxene is often abundant. Unit 2 pillows with plagioclase and olivine microphenocrysts in their glassy margins have interiors composed of felty mats of plagioclase laths in a fine-grained groundmass of cryptocrystalline pyroxene and plagioclase. In a few samples, pyroxene laths are intergrown with the plagioclase laths. In unit 3, where the microphenocryst assemblage in the glassy 
zone is either plagioclase and pyroxene (3A) or plagioclase-pyroxene-olivine (3B), both pyroxene and olivine are equally well crystallized, pyroxene generally occurring as granules, rather than laths; typically, the texture is either intergranular or intersertal, with a devitrifiedglass matrix. The only feature of these pillow basalts texturally atypical of MORB is the high vesicularity.

\section{Vesicles}

The most distinctive feature of the Site 442 basalts is their large vesicle content. Generally, two distinct vesicle-size populations can be distinguished. Large $(0.5-3.0 \mathrm{~mm})$ vesicles are present in highly variable proportion ( $1-5 \%$ by volume) and range in shape from perfectly round to highly irregular. Small $(0.2 \mathrm{~mm})$ vesicles are much more abundant and when present in great amounts (up to $37 \%$ ) give the rock a "foam-like", appearance in thin section. These vesicles are typically larger (avg. $0.47 \mathrm{~mm}$ ) and fewer in number in the massive cooling units than in the pillow lavas. The average vesicularity of Site 442 basalts is 16.31 volume per cent $(1 \mathrm{~s}=7.4 \mathrm{vol}$. $\%)$, based on visual estimates from 51 thin sections. These estimates were checked and corrected for a slight bias in visual estimation by point counting 10 of the thin sections.

While the interiors of both sills and pillows are highly vesicular, the clear glassy chill zones, with a few exceptions, contain less than 1 per cent very small vesicles. These frequently have numerous small sulfide blebs on their walls. The few vesicles in the glass zones suggest that, while a free gas phase was not abundant prior to eruption, the lavas were already saturated with respect to volatiles, including sulfur.

\section{Alteration}

There is a large difference in the degree of alteration of the upper massive basalts and the lower pillow-lava units. Emerald-green, pale-brown, and brown pleochroic clays (smectites?) are abundant and are often well crystallized in relatively coarse plates and mats in the massive basalts. Amygdules with clay or sparry-calcite fillings are common. In the interiors of the thicker subunits ( $1 \mathrm{E}$ and $1 \mathrm{~K})$, olivine is represented entirely by green-clay pseudomorphs, while in the remaining units and in the margins of the thicker units it is represented by calcite pseudomorphs. Curiously, small octahedral spinel grains are completely unaltered in the calcite pseudomorphs. In the interior of sub-unit $1 \mathrm{~K}$, plagioclase is partially saussuritized, and a fibrous, acicular, high-relief mineral (actinolite?) occurs in the altered groundmass.

The pillow basalts-except for a little alteration which has produced some amorphous, brown, intergranular clay and some palagonitization of the margins of the glassy chill zones-show little evidence of alteration. Olivine is fresh and completely unaltered in most cases, or shows a little iddingsitization, typical of slightly altered submarine pillow basalts.

The logical explanation for the greater alteration of the massive basalts is deuteric alteration accompanying relatively slow cooling after intrusion into wet, semi- consolidated or unconsolidated sediments. The relatively unaltered pillow basalts, then, probably reflect rapid cooling which accompanied extrusion on the open-ocean floor.

\section{Site 443}

\section{Stratigraphy}

At Site 443, the uppermost basalt unit is unique, consisting of a single glassy fragment of plagioclaseclinopyroxene phyric basalt. In the remaining basalt section, 41 meters of plagioclase and olivine phyric massive basalts and diabases are underlain by 82 meters of intercalated aphyric vesicular pillow basalts and plagioclase and olivine phyric massive basalts. Beneath three of the pillow-basalt sub-units (3A, 3C, 5D) are massive aphyric basalts (sub-units $3 \mathrm{~B}, 3 \mathrm{D}, 5 \mathrm{E}$ ) which are petrographically similar to the overlying pillows, and have similar vesicularities, chemistries, and magnetic inclinations. Each of these may be the massive basal portion of a large, composite lava flow whose basal part ran out under a carapace of its own pillows or a consanguineous sheet flow underlying the pillow lava. Nannofossil chalk and limestone were recovered from two intervals: $6 \mathrm{~cm}$ (actual recovery), at the top of the pillow basalts in Core 58 ; and $12 \mathrm{~cm}$, at the top of a very thin unit in Core 59, which may be either a sheet flow or pillow basalt.

As at Site 442, glassy chill zones are abundant in the pillow-lava units (42 were found in the less than 9.82 meters of pillow basalt actually recovered). These glassy rinds are randomly oriented and are morphologically typical of pillow basalts.

Chill zones are less common in the massive basalts and are generally horizontal in the recovered section. They rarely exhibit a thin glass zone, and usually consist of a sharp gradation from fine-grained to aphanitic or glassy basalt. Most of the glass-rich zones in the massive basalts come from aphyric basalts in Cores 59 and 61 (sub-units $3 \mathrm{~F}, 3 \mathrm{G}, 3 \mathrm{H}, 3 \mathrm{I}, 5 \mathrm{~A}$, and 5B). Given the frequency of the glass zones, the small size of the glass-rich massive units, and their petrographic similarity to the pillow basalt units, we feel that these units are also extrusives, although they may be either pillow basalts or sheet flows.

The presence of nannofossil chalk and limestone directly above one of these aphyric, glass-rich, massive units (3F) also suggests an extrusive origin. Similar limestones are common as coatings on dredged rubble and pillows. Elsewhere, at both Sites 442 and 443, such limestones are present only with pillow basalts. They are likely the simple product of recrystallization and induration of calcareous sediment locally precipitated by chemical reactions accompanying halmyrolysis of the underlying basic igneous rock. In the absence of any indication of upfaulting and brecciation, such limestones appear to be excellent indicators of an extrusive origin of the underlying basalt, their presence suggesting only a thin veneer or local ponding of calcareous sediment in depressions and interstices of pillows.

Accordingly, we divide the stratigraphic section at Site 443 into aphyric, vesicular, pillow basalts; extrusive flows; and phyric, non-vesicular, massive basalts and 
diabase, some of which may have been intruded into or above the pillow-lava sequence. Their low vesicularity and high phenocryst content suggest extensive intratelluric crystallization and degassing of the phyric basalts in a shallow magma chamber prior to intrusion or extrusion.

\section{Petrography}

The Site 443 pillow lavas, massive basalts, and diabases are texturally similar to those from Site 442 . Modal analyses are given in Table 2. Differences are due largely to the low vesicularity of many of the Site 443 massive basalts and their greater phenocryst contents. Pyroxene ranges from clear to pale brown. It is generally interstitial to olivine and plagioclase. Olivine is generally euhedral to subhedral and ranges from aphanitic groundmass to $5 \mathrm{~mm}$. Plagioclase is the most common phenocryst and groundmass phase. Large phenocrysts are often resorbed and have sieve texture. Oscillatory zoning of plagioclase phenocrysts is very common. Titanomagnetite is ubiquitous, occurring interstitially as granules, laths, or skeletal grains. Spinel is less common than at Site 442 and is found as small octahedral inclusions in olivine and in calcite pseudomorphs after olivine, where it is again unaltered.

The clinopyroxene-plagioclase microphyric basalt (unit 1) contains numerous clear, euhedral clinopyroxene microphenocrysts which are generally unzoned but have typical undulatory extinction. In addition, the basalt contains abundant relatively coarse-grained (2-5 $\mathrm{mm})$ plagioclase phenocrysts and glomerocrysts with sharp euhedral outlines in the aphanitic glassy matrix and oscillatory zoning.

A few sediment inclusions were found. One, in Sample 54-5, 46-148 cm, consists of a highly convoluted patch of sparry calcite enclosed in a selvage of palagoni- tized and devitrified glass in otherwise crystalline basalt. A 12 by $5 \mathrm{~mm}$, rectangular calcite fragment in Sample $57-2,125-127 \mathrm{~cm}$ contains polygonally interlocking sparry-calcite spherulites with interstitial palagonite. There is a fine-grained selvage of basalt around the fragment, again suggesting some local chilling of the basalt. The first fragment comes from a massive basalt, the second from the interior of a basalt pillow.

\section{Alteration}

As at Site 442, there are differences in alteration of the pillow basalts and massive basalts. Generally speaking, olivine is better preserved than at Site 442 , being completely altered in only 29 of 84 examined thin sections, and at least some olivine is preserved in every subunit except 3B. In pillow basalts, only calcite has formed pseudomorphs after olivine; in the massive cooling units, calcite has formed pseudomorphs after olivine at the margins, and smectite and clay have formed pseudomorphs after olivine in the interiors. As at Site 442 , the clay ranges from cryptocrystalline, yellow clay to relatively coarsely crystalline, tabular, micaceous plates; these may be strongly pleochroic (pale green to clear), and they have a high birefringence which distinguishes them from chlorite. They are distinguished from talc by their lower birefringence (up to 0.034 , as opposed to 0.045 ). We believe that this mineral may be smectite. In one sample $(61-4,106-108 \mathrm{~cm})$ from the interior of a massive unit, the primary replacement mineral may be serpentine, rather than clay. As at Site 442, many of the massive units are crisscrossed by carbonate veins; much of the carbonate is biaxial. The difference in alteration between pillows and massive basalts may reflect deuteric alteration of the more slowly cooled massive units-as opposed to simple halmyrolysis of the pillow basalts.

TABLE 2

Modal Analyses of Representative Hole 443 Basalts and Diabases

\begin{tabular}{|c|c|c|c|c|c|c|c|}
\hline \multirow[b]{2}{*}{ Component } & \multicolumn{7}{|c|}{ Interval and Rock Type } \\
\hline & $\begin{array}{c}49-3, \\
59-52 \\
\text { basalt }\end{array}$ & $\begin{array}{r}50-2 \\
14-19 \\
\text { diabase }\end{array}$ & $\begin{array}{c}57-2 \\
100-107\end{array}$ & $\begin{array}{c}60-6 \\
100-107 \\
\text { diabase }\end{array}$ & $\begin{array}{r}62-3, \\
\text { glassy zone }\end{array}$ & $\begin{array}{l}-125, \\
\text { crypto- } \\
\text { crystalline }\end{array}$ & $\begin{array}{c}64-2 \\
100-104\end{array}$ \\
\hline Matrix & 74.6 & 7.2 & 97.6 & 3.5 & $3.3^{\mathrm{b}}$ & 92.8 & 1.0 \\
\hline Vesicles & 5.4 & 1.7 & 1.4 & - & 1.2 & 6.8 & - \\
\hline Glass & - & - & - & - & 94.3 & - & - \\
\hline \multicolumn{8}{|c|}{ Groundmass or microphenocrysts } \\
\hline Plagioclase & 7.5 & 43.4 & - & 50.0 & 0.6 & - & 45.7 \\
\hline Pyroxene & 1.7 & 28.7 & - & 24.8 & - & - & 21.6 \\
\hline Olivine & - & 1.2 & 1.0 & 2.2 & 0.6 & - & 26.9 \\
\hline Relict Olivine & - & $\mathrm{nc}$ & - & 17.0 & - & - & 2.0 \\
\hline Opaque & $\mathrm{nc}^{\mathrm{a}}$ & 6.3 & $\mathrm{nc}$ & 2.6 & - & $\mathrm{nc}$ & 2.7 \\
\hline \multicolumn{8}{|l|}{ Phenocrysts } \\
\hline Plagioclase & 10.5 & 11.5 & - & - & - & - & - \\
\hline Pyroxene & 0.3 & - & - & - & - & 0.4 & - \\
\hline Olivine & - & - & - & - & - & - & - \\
\hline Relict Olivine & - & - & - & - & - & - & - \\
\hline Points Counted & 1433 & 1559 & 1922 & 1905 & 335 & 1669 & 1351 \\
\hline
\end{tabular}

a "nc" - not counted.

bicrolites. 


\section{Site 444}

\section{Stratigraphy}

At least three massive cooling units were drilled at Site 444. Nineteen meters of sediment underlies the first of these units, while a single sediment fragment was recovered between the lower two. The upper unit is an alkali basalt, and the lower two are olivine tholeiites.

Although the contacts of the alkali basalt were not recovered, the sediment immediately above and below the unit is an anomalous dark, chocolate color which we believe to be an effect of intrusion of the basalt into the sediment. This and the strong differentiation of the basalt lead us to conclude that it is a sill. The organic-carbon content of the sediment where measured below the sill is anomalously high and is attributed to baking of the organic carbon prior to completion of bacterial degradation. The preserved carbon content suggests that the basalt was intruded into sediment 0.7 to $2.0 \mathrm{~m}$.y. old (Waples, this volume).

The lower two units are also believed to be sills. While similar in appearance, the distinctly different composition of their glassy chill zones clearly indicates that they are separate intrusions. The upper chill zone of the upper olivine tholeiite has a ropey pahoehoe surface with a thin white coating which appears to be baked sediment, indicating that the sill can also be interpreted as a sub-sediment flow.

\section{Petrography}

The upper sill is an analcite-bearing alkali olivine basalt. The essential constituents (Table 3 ) are calcic plagioclase, titaniferous augite or salite, olivine, anorthoclase, analcite, and iron oxides. Biotite and kaersutite are present in some thin sections. Apatite and spinel are present as accessory minerals. Zeolites including phillipsite and natrolite are common and often form ray-like masses several millimeters across in the groundmass. These masses may be intergrown with analcite and other secondary minerals and may replace nepheline. Other secondary minerals, including clays (smectite?), chlorite (diabantite), talc, and actinolite are abundant. In some thin sections, small, dark-green grains are found as late groundmass phases and as late overgrowths on kaersu-

TABLE 3

Modes of Site 444 Basalts

\begin{tabular}{lcccc}
\hline & \multicolumn{2}{c}{ Upper Sill } & & Lower Sills \\
\cline { 2 - 3 } & $20-1,62-64 \mathrm{~cm}$ & $20-3,30-32 \mathrm{~cm}$ & & $26-3,0-4 \mathrm{~cm}$ \\
\hline Matrix & 20.7 & 32.2 & 11.0 \\
Plagioclase & 38.5 & 28.0 & 52.9 \\
Pyroxene & 7.7 & 12.1 & 21.7 \\
Olivine & 0.6 & 11.4 & 9.7 \\
K-Spar & 10.2 & 0.3 & - \\
Hornblende & 5.6 & 0.2 & - \\
Analcite & 10.3 & 13.7 & \\
Oxide & 6.5 & 1.7 & \\
Apatite* & 1 & 1 & 3.0 \\
Vesicules* & 1 & 1 & tr \\
Spinel* & - & 0.1 & 1.7 \\
Points Counted & 1024 & 1032 & - \\
\hline
\end{tabular}

*Visual estimate. tite and augite; these could be either fluor-amphiboles or aegirine-augite.

The plagioclase in the alkali basalt is often strongly zoned and is either interstitial to idiomorphic pyroxene or intergrown with large, ophitic pyroxene granules or laths. In the groundmass, plagioclase occurs either as acicular laths or as box-like quench crystals in a mass of clay, zeolites, and analcite. In some thin sections, the plagioclase is partially saussuritized. While the pyroxene does not appear to be a major groundmass phase, larger (1-2 $\mathrm{mm})$ grains are abundant and are generally strongly zoned, often with hourglass structure. The pyroxene ranges from clear to pale brown near the base and top of the sill, to a rose color in the kaersutite-bearing diabase in the upper middle of the sill, indicating titaniferous augite. Olivine is present as 0.25 to $0.75 \mathrm{~mm}$ euhedra and subhedra and is largely replaced by green-brown clay, chlorite, or talc. In a few instances, hair-like, palegreen actinolite(?) needles appear to be part of the replacement. Titanomagnetite is abundant and generally interstitial, and occurs as granules, small euhedra, laths, or skeletal crystals. Kaersutite is an accessory phase in most thin sections, and a principal late phase in some, either as prisms or as overgrowths on rose-colored augite. Late interstitial biotite occurs in the place of kaersutite in some thin sections. Analcite is abundant in most thin sections, replacing plagioclase along cracks or present as clots and cryptocrystalline masses in the groundmass. Anorthoclase is present as large interstitial masses or overgrowths on plagioclase grains.

The upper sill generally has porphyritic to diabasic texture, depending on the amount of replaced primary olivine and groundmass (10-30\%). The sequence of crystallization was clearly olivine $\rightarrow$ pyroxene (or pyroxene-plagioclase) $\rightarrow$ plagioclase $\rightarrow$ kaersutite $\rightarrow$ titanomagnetite $\rightarrow$ anorthoclase $\rightarrow$ apatite and analcite. The analcite is either late magmatic or deuteric.

The proportions of the primary phases vary considerably through the sill: anorthoclase, plagioclase, titanomagnetite, apatite, and hydrous phases are noticeably enriched near the top, and olivine and pyroxene are more abundant near the base (Table 3 ). As is the case in many such alkaline sills, considerable in situ differentiation has occurred (e.g., Kuno, 1968).

The lower sills (2A and 2B) are plagioclase-olivine phyric basalt and diabase. The plagioclase phenocrysts are stubby crystals 2 to $3 \mathrm{~mm}$ across and frequently zoned; they are generally euhedral in the chill zone, but commonly subhedral and resorbed in the interior of the flow. A single rounded 2-mm pyroxene phenocryst was found in the upper chill zone. The groundmass in sill $2 \mathrm{~A}$ consists of 1 to $4 \mathrm{~mm}$ pyroxene clots ophitically enclosing euhedral plagioclase laths; interstitial olivine and titanomagnetite are present in lesser amounts. The lower sill (2B) contains subophitic diabase with more abundant olivine. Generally in the lower sill olivine is nearly equal to pyroxene in abundance. Crystal settling and in situ differentiation may have occurred in the unit 2 sill, although with somewhat less dramatic effects than in unit 1 , as the proportions of both olivine and plagioclase appear to vary through the two sills. 


\section{MINERALOGY}

\section{Analytical Technique}

All mineral analyses were done on a Materials Analysis Corporation electron microprobe at the Department of Earth and Planetary Sciences, Massachusetts Institute of Technology. Fifteen kilovolts operating voltage and [39] amps flag current were used. Mineral standards were used for all analyses. The quality of the analysis varies with the analyzed mineral and the standardization; we believe that analyses for olivine and pyroxene are of high quality, those for plagioclase are of fair quality, and those for the remaining minerals (anorthoclase, hornblende, biotite, analcite, zeolites) all are of uncertain quality. The raw data were corrected using the Bence-Albee method, and an internal drift correction was made by periodic re-analysis of a selected standard mineral grain between unknowns to obtain a projected linear drift correction for each element at the time of measurement on the unknown. The analytical results are included in Tables 4 through 16, and the major compositional parameters of pyroxene, plagioclase, and olivine are plotted in Figure 2.

\section{Olivine}

Olivine from three widely spaced Site 442 pillows shows little variation in composition $\left(\mathrm{Fo}_{82.3}-\mathrm{Fo}_{82.9}\right)$, while that in Site 443 pillows and sills has a larger variation $\left(\mathrm{Fo}_{81.3}-\mathrm{Fo}_{86.6}\right)$. The variation within the Site 443 sills may reflect progressive in situ fractional crystallization. All these compositions lie well within the range of abyssal tholeiites from the Atlantic and Pacific. The olivine from the analcite-bearing alkali olivine basalt sill at Site 444 is similar to that found in the Site 442 and 443 basalts. Olivine from the lower Site 444 sills is fayalitic relative to that in most abyssal tholeiites and has a relatively large range of composition. This probably reflects considerable fractionation of the sills.

Plotted in Figure 3 is the forsterite content of olivine versus the molar ratio $\mathrm{Fe} /(\mathrm{Mg}+\mathrm{Fe})$ of coexisting glass from pillow-basalt rinds. Also plotted are XRF wholerock compositions against olivine compositions from the Site 443 and 444 sills. Lines connect the points for the sills to the compositions of glass in the overlying chill zone, where data are available. Also plotted in Figure 3 are the range of possible equilibrium compositions for different $\mathrm{Mg}-\mathrm{Fe}$ distribution coefficients $\left(K_{\mathrm{D}}\right)$ for melt and coexisting olivine, where all iron is ferrous. Roeder and Emslie's (1970) study of these equilibria suggests that $K_{\mathrm{D}}$ is equal to 0.300 and is relatively insensitive to variations in temperature, pressure, and melt composition. Bender et al. (1978) measured $K_{\mathrm{D}}$ of a Mid-Atlantic Ridge basalt under a range of conditions and suggested both a temperature dependence (at $0 \mathrm{~kb}$, $K_{\mathrm{D}}{ }^{1280^{\circ} \mathrm{C}}=0.30$; at $10 \mathrm{~kb}, K_{\mathrm{D}}{ }^{1290^{\circ} \mathrm{C}}=0.31$ ) and a small pressure dependence. Unfortunately, Bender et al. neither controlled oxygen fugacity nor measured ferric iron. Accordingly, there is some ambiguity in their measurements. Direct measurement of $K_{\mathrm{D}}$ in natural abyssal basalt glasses, assuming that all iron is ferrous, gives apparent distribution coefficients $\left(K^{\prime}\right.$ D) close to 0.27 (e.g.,

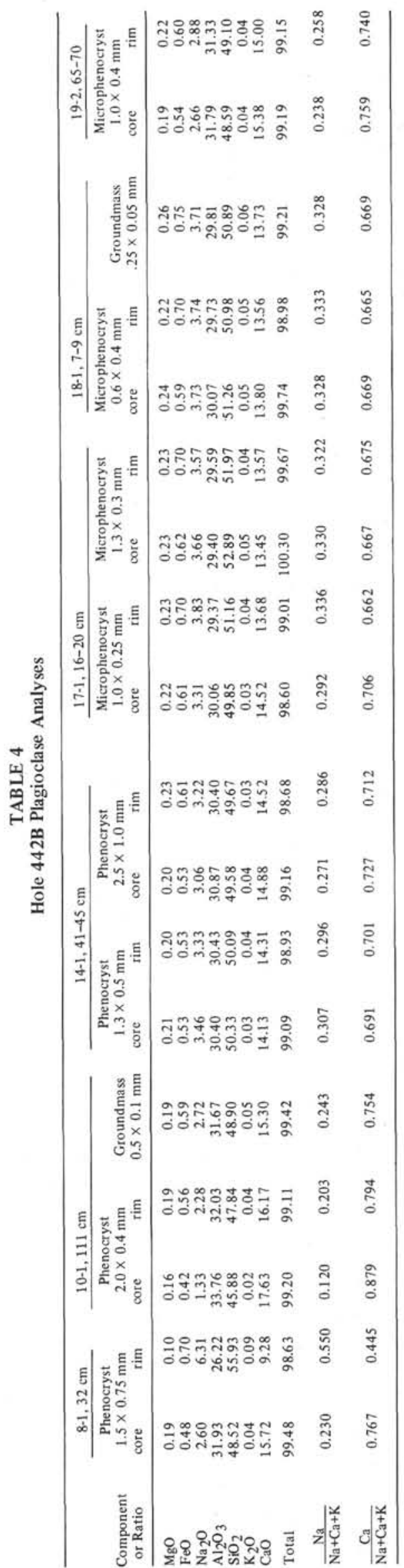


TABLE 5

Hole 442B Pyroxene Analyses

\begin{tabular}{|c|c|c|c|c|c|c|}
\hline \multirow[b]{2}{*}{$\begin{array}{l}\text { Component } \\
\text { or Ratio }\end{array}$} & \multirow{2}{*}{$\begin{array}{c}\frac{8-1,32 \mathrm{~cm}}{\text { Phenocryst }} \\
2.5 \times 2.0 \mathrm{~mm}\end{array}$} & \multirow{2}{*}{$\begin{array}{c}\frac{10-1,111 \mathrm{~cm}}{\text { Groundmass }} \\
0.25 \times 0.25 \mathrm{~mm}\end{array}$} & \multirow{2}{*}{$\begin{array}{c}\frac{17-1,16-20 \mathrm{~cm}}{\text { Microphenocryst }} \\
0.5 \times 0.4 \mathrm{~mm}\end{array}$} & \multicolumn{2}{|c|}{$18-1,7-9 \mathrm{~cm}$} & \multirow{2}{*}{$\begin{array}{c}\frac{19-2,65-70 \mathrm{~cm}}{\text { Microphenocryst }} \\
0.6 \times 0.4 \mathrm{~mm}\end{array}$} \\
\hline & & & & $\begin{array}{l}\text { Microphenocryst } \\
0.6 \times 0.4 \mathrm{~mm}\end{array}$ & $\begin{array}{c}\text { Microphenocryst } \\
3 \times 0.3 \mathrm{~mm}\end{array}$ & \\
\hline $\mathrm{MgO}$ & 16.41 & 14.86 & 16.80 & 16.89 & 15.98 & 16.81 \\
\hline $\mathrm{FeO}$ & 5.24 & 7.49 & 7.28 & 6.50 & 6.64 & 5.42 \\
\hline $\mathrm{Na}_{2} \mathrm{O}$ & 0.26 & 0.28 & 0.33 & 0.20 & 0.28 & 0.23 \\
\hline $\mathrm{Al}_{2} \mathrm{O}_{3}$ & 2.67 & 5.01 & 3.54 & 3.39 & 3.88 & 2.60 \\
\hline $\mathrm{SiO}_{2}$ & 52.32 & 50.16 & 51.18 & 51.24 & 51.17 & 51.87 \\
\hline $\mathrm{K}_{2} \mathrm{O}$ & 0.01 & 0.02 & 0.00 & 0.01 & 0.01 & .00 \\
\hline $\mathrm{CaO}$ & 21.62 & 20.75 & 19.25 & 19.79 & 20.68 & 20.97 \\
\hline $\mathrm{TiO}_{2}$ & 0.58 & 1.18 & 0.87 & 0.88 & 0.90 & 0.55 \\
\hline $\mathrm{Cr}_{2} \mathrm{O}_{3}$ & 0.40 & 0.07 & 0.09 & 0.11 & 0.15 & 0.21 \\
\hline $\mathrm{MnO}$ & 0.16 & 0.19 & 0.27 & 0.19 & 0.16 & 0.14 \\
\hline Total & 99.67 & 100.01 & 99.61 & 99.20 & 99.85 & 98.80 \\
\hline$\frac{\mathrm{Mg}}{\mathrm{Mg}+\mathrm{Fe}}$ & 0.848 & 0.780 & 0.805 & 0.823 & 0.811 & 0.847 \\
\hline$\frac{\mathrm{Mg}}{\mathrm{Mg}+\mathrm{Fe}+\mathrm{Ca}}$ & 0.471 & 0.438 & 0.484 & 0.486 & 0.463 & 0.482 \\
\hline$\frac{\mathrm{Ca}}{\mathrm{Mg}+\mathrm{Fe}+\mathrm{Ca}}$ & 0.445 & 0.439 & 0.398 & 0.409 & 0.430 & 0.431 \\
\hline
\end{tabular}

TABLE 6

Hole 442B Olivine Analyses

\begin{tabular}{|c|c|c|c|}
\hline $\begin{array}{l}\text { Component } \\
\text { or Ratio }\end{array}$ & $\begin{array}{l}\frac{14-1,41-45 \mathrm{~cm}}{\text { Phenocryst }} \\
1 \times 0.6 \mathrm{~mm}\end{array}$ & $\begin{array}{c}\frac{17-1,16-20 \mathrm{~cm}}{\text { Phenocryst }} \\
0.9 \times 0.6 \mathrm{~mm}\end{array}$ & 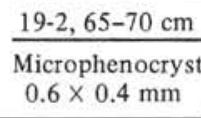 \\
\hline $\mathrm{MgO}$ & 43.51 & 42.97 & 46.34 \\
\hline $\mathrm{FeO}$ & 15.99 & 16.38 & 17.73 \\
\hline $\mathrm{SiO}_{2}$ & 39.96 & 39.42 & $?$ \\
\hline $\mathrm{CaO}$ & 0.25 & 0.26 & $?$ \\
\hline $\mathrm{MnO}$ & 0.21 & 0.24 & 0.25 \\
\hline $\mathrm{Al}_{2} \mathrm{O}_{3}$ & 0.04 & 0.07 & 0.08 \\
\hline $\mathrm{Ti}_{2}$ & 0.00 & 0.01 & 0.00 \\
\hline $\mathrm{Cr}_{2} \mathrm{O}_{3}$ & 0.00 & 0.00 & 0.07 \\
\hline Total & 99.96 & 99.42 & $?$ \\
\hline \multicolumn{4}{|l|}{$\mathrm{Mg}$} \\
\hline$\overline{\mathrm{Mg}+\mathrm{Fe}}$ & 0.829 & 0.824 & 0.823 \\
\hline
\end{tabular}

Dungan et al., 1978). Shown in Figure 3 are data for basalt olivine-glass pairs from Dungan et al. (1978) for samples from near the Mid-Atlantic Ridge at $23^{\circ} \mathrm{N}$. We note that if only 8 per cent of the total iron in these sam- ples is ferric, $K_{\mathrm{D}}$ will equal 0.300 . Thus, it appears reasonable that $K_{\mathrm{D}}$ is close to 0.300 at near-liquidus conditions and low pressures, for most abyssal basalts.

Significant upward deviations of olivine-glass pairs in Figure 3 from the curve $K_{\mathrm{D}}=0.300$ should indicate high ferric-iron contents. The apparent $K_{\mathrm{D}}$ of the Shiko$\mathrm{ku}$ Basin olivine-glass pairs is very low $\left(K_{\mathrm{D}}{ }^{\prime}=0.227\right)$ and indicates that these basalts had primary ferric-iron contents much higher at the time of eruption than those typical of mid-ocean-ridge basalts. If high ferric-iron contents are indicators of high water fugacities as well as high oxygen contents (e.g., Kennedy, 1948), this observation would be consistent with the high vesicularity of the Site 442 and 443 pillow basalts and a high initial water content.

Downward deviations of whole-rock-olivine pairs in Figure 3 is likely to reflect significant olivine accumulation, and hence a bulk-rock composition too magnesian to represent the actual equilibrium. It is interesting to note that the olivine in one Site 443 sill appears to be in equilibrium with the liquid composition represented by

TABLE 7

Hole 443 Plagioclase Analyses

\begin{tabular}{|c|c|c|c|c|c|c|c|c|c|c|c|c|c|c|c|c|}
\hline \multirow{3}{*}{$\begin{array}{l}\text { Compo- } \\
\text { nent or } \\
\text { Ratio }\end{array}$} & \multicolumn{4}{|c|}{$49-3,59-62 \mathrm{~cm}$} & \multirow{2}{*}{\multicolumn{2}{|c|}{$\begin{array}{c}\frac{50-2,14-19 \mathrm{~cm}}{\text { Phenocryst }} \\
3.5 \times 3.5 \mathrm{~mm}\end{array}$}} & \multicolumn{4}{|c|}{$60-6,102-107 \mathrm{~cm}$} & \multirow{2}{*}{\multicolumn{2}{|c|}{$\frac{62-3,121-125 \mathrm{~cm}}{\text { Microphenocryst }}$}} & \multicolumn{4}{|c|}{$64-2,100-104 \mathrm{~cm}$} \\
\hline & \multicolumn{2}{|c|}{$\begin{array}{l}\text { Phenocryst } \\
3.0 \times 1.5 \mathrm{~mm}\end{array}$} & \multicolumn{2}{|c|}{$\begin{array}{c}\text { Microphenocryst } \\
0.6 \times 0.4 \mathrm{~mm}\end{array}$} & & & \multicolumn{2}{|c|}{$\begin{array}{l}\text { Phenocryst } \\
1.5 \times 1.3 \mathrm{~mm}\end{array}$} & \multicolumn{2}{|c|}{$\begin{array}{c}\text { Groundmass } \\
1.3 \times 0.2 \mathrm{~mm}\end{array}$} & & & \multicolumn{2}{|c|}{$\begin{array}{c}\text { Phenocryst } \\
5.0 \times 4.0 \mathrm{~mm}\end{array}$} & \multicolumn{2}{|c|}{$\begin{array}{c}\text { Groundmass } \\
0.6 \times 0.2 \mathrm{~mm}\end{array}$} \\
\hline & core & rim & cote & rim & core & rim & core & rim & core & rim & core & rim & core & rim & core & rim \\
\hline Mgo & 0.21 & 0.23 & 0.23 & 0.26 & 0.20 & 0.17 & 0.21 & 0.21 & 0.24 & 0.08 & 0.22 & 0.52 & 0.18 & 0.18 & 0.23 & 0.25 \\
\hline $\mathrm{FeO}$ & 0.41 & 0.54 & 0.53 & 0.62 & 0.40 & 0.40 & 0.42 & 0.53 & 0.49 & 0.63 & 0.40 & 0.93 & 0.32 & 0.55 & 0.42 & 0.47 \\
\hline $\mathrm{Na}_{2} \mathrm{O}$ & 2.10 & 3.26 & 2.86 & 3.86 & 1.68 & 5.17 & 3.18 & 3.76 & 3.70 & 6.28 & 2.68 & 2.24 & 1.50 & 3.75 & 2.91 & 2.60 \\
\hline $\mathrm{Al}_{2} \mathrm{O}_{3}$ & 32.77 & 31.30 & 31.93 & 30.26 & 33.84 & 28.51 & 31.09 & 30.43 & 30.44 & 27.05 & 32.04 & 31.77 & 33.84 & 30.50 & 31.70 & 32.13 \\
\hline $\mathrm{SiO}_{2}$ & 48.05 & 50.30 & 49.30 & 51.43 & 47.03 & 54.36 & 49.93 & 50.72 & 50.60 & 55.93 & 49.02 & 47.81 & 46.58 & 51.08 & 49.76 & 49.05 \\
\hline $\mathrm{K}_{2} \mathrm{O}^{\circ}$ & 0.03 & 0.03 & 0.04 & 0.06 & 0.03 & 0.10 & 0.08 & 0.03 & 0.04 & 0.07 & 0.03 & 0.03 & 0.01 & 0.03 & 0.04 & 0.03 \\
\hline $\mathrm{CaO}$ & 16.46 & 14.57 & 15.37 & 13.69 & 17.24 & 11.31 & 14.76 & 14.11 & 14.17 & 9.97 & 15.62 & 16.38 & 17.40 & 13.83 & 14.99 & 15.58 \\
\hline Total & 100.03 & 100.23 & 100.26 & 100.18 & 100.42 & 100.00 & 99.67 & 99.79 & 99.68 & 100.01 & 100.01 & 99.68 & 99.83 & 99.92 & 100.05 & 100.11 \\
\hline$\frac{\mathrm{Na}}{\mathrm{Na}+\mathrm{Ca}+\mathrm{K}}$ & 0.188 & 0.288 & 0.252 & 0.337 & 0.150 & 0.451 & 0.280 & 0.325 & 0.321 & 0.531 & 0.237 & 0.198 & 0.135 & 0.329 & 0.260 & 0.232 \\
\hline$\frac{\mathrm{Ca}}{\mathrm{Na}+\mathrm{Ca}+\mathrm{K}}$ & 0.811 & 0.710 & 0.746 & 0.659 & 0.848 & 0.543 & 0.716 & 0.673 & 0.677 & 0.465 & 0.761 & 0.800 & 0.864 & 0.669 & 0.738 & 0.766 \\
\hline
\end{tabular}


TABLE 8

Hole 443 Pyroxene Analyses

\begin{tabular}{|c|c|c|c|c|c|}
\hline \multirow[b]{2}{*}{$\begin{array}{l}\text { Component } \\
\text { or Ratio }\end{array}$} & \multirow{2}{*}{$\begin{array}{c}\frac{49-3,59-62 \mathrm{~cm}}{\text { Microphenocryst }} \\
0.5 \times 0.4 \mathrm{~mm}\end{array}$} & \multirow{2}{*}{$\frac{50-2,14-19 \mathrm{~cm}}{\text { Groundmass }}$} & \multicolumn{2}{|c|}{$60-6,102-107 \mathrm{~cm}$} & \multirow{2}{*}{$\begin{array}{c}\frac{64-2,100-104 \mathrm{~cm}}{\text { Groundmass }} \\
1.2 \times 0.3 \mathrm{~mm}\end{array}$} \\
\hline & & & $\begin{array}{l}\text { Groundmass } \\
1.25 \times 0.9 \mathrm{~mm}\end{array}$ & $\begin{array}{l}\text { Groundmass } \\
0.7 \times 0.6 \mathrm{~mm}\end{array}$ & \\
\hline $\mathrm{MgO}$ & 15.87 & 14.80 & 12.75 & 12.71 & 16.13 \\
\hline $\mathrm{FeO}$ & 6.03 & 9.62 & 13.38 & 13.18 & 8.20 \\
\hline $\mathrm{Na}_{2} \mathrm{O}$ & 0.36 & 0.30 & 0.36 & 0.38 & 0.30 \\
\hline $\mathrm{Al}_{2} \mathrm{O}_{3}$ & 4.57 & 3.02 & 2.39 & 2.43 & 2.50 \\
\hline $\mathrm{SiO}_{2}$ & 50.29 & 50.24 & 50.11 & 49.83 & 52.43 \\
\hline $\mathrm{K}_{2} \mathrm{O}$ & 0.01 & 0.00 & 0.00 & 0.00 & 0.00 \\
\hline $\mathrm{CaO}$ & 19.90 & 19.75 & 18.53 & 18.96 & 19.74 \\
\hline $\mathrm{TiO}_{2}$ & 0.92 & 1.19 & 1.41 & 1.39 & 0.89 \\
\hline $\mathrm{Cr}_{2} \mathrm{O}_{3}$ & 1.00 & 0.05 & 0.00 & 0.00 & 0.19 \\
\hline $\mathrm{MnO}$ & 0.25 & 0.61 & 0.60 & 0.59 & 0.28 \\
\hline Total & 99.20 & 99.58 & 99.53 & 99.47 & 100.66 \\
\hline$\frac{\mathrm{Mg}}{\mathrm{Mg}+\mathrm{Fe}}$ & 0.824 & 0.773 & 0.630 & 0.632 & 0.778 \\
\hline$\frac{\mathrm{Mg}}{\mathrm{Mg}+\mathrm{Fe}+\mathrm{Ca}}$ & 0.473 & 0.431 & 0.380 & 0.377 & 0.462 \\
\hline$\frac{\mathrm{Ca}}{\mathrm{Mg}+\mathrm{Fe}+\mathrm{Ca}}$ & 0.426 & 0.412 & 0.396 & 0.404 & 0.406 \\
\hline
\end{tabular}

TABLE 9

Hole 443 Olivine Analyses

\begin{tabular}{|c|c|c|c|c|c|c|c|c|}
\hline \multirow{3}{*}{$\begin{array}{c}\text { Component } \\
\text { or Ratio }\end{array}$} & \multicolumn{2}{|c|}{$50-2,14-19 \mathrm{~cm}$} & \multicolumn{2}{|c|}{$57-2,100-107 \mathrm{~cm}$} & \multicolumn{2}{|c|}{$60-6,102-107 \mathrm{~cm}$} & \multirow{3}{*}{$\begin{array}{c}\frac{62-3,121-125 \mathrm{~cm}}{\text { Phenocryst }} \\
1.25 \times 0.6 \mathrm{~mm}\end{array}$} & \multirow{3}{*}{$\begin{array}{c}\frac{64-2,100-104 \mathrm{~cm}}{\text { Phenocryst }} \\
1.5 \times 1.4 \mathrm{~mm}\end{array}$} \\
\hline & \multicolumn{2}{|c|}{ Groundmass } & \multicolumn{2}{|c|}{ Microphenocrysts } & \multirow{2}{*}{\multicolumn{2}{|c|}{$\begin{array}{c}\text { Groundmass } \\
1.25 \times 0.7 \mathrm{~mm}\end{array}$}} & & \\
\hline & $0.5 \times 0.4 \mathrm{~mm}$ & $0.4 \times 0.4 \mathrm{~mm}$ & $0.6 \times 0.4 \mathrm{~mm}$ & $0.3 \times 0.2 \mathrm{~mm}$ & & & & \\
\hline $\mathrm{MgO}$ & 44.78 & 42.96 & 44.40 & 43.88 & 46.74 & 45.81 & 46.75 & 46.59 \\
\hline $\mathrm{FeO}$ & 15.47 & 17.62 & 15.65 & 15.72 & 12.94 & 14.22 & 13.06 & 13.16 \\
\hline $\mathrm{SiO}_{2}$ & 39.07 & 38.86 & 39.13 & 39.03 & 39.62 & 39.64 & 39.58 & 39.79 \\
\hline $\mathrm{CaO}$ & 0.29 & 0.30 & 0.21 & 0.25 & 0.28 & 0.30 & 0.26 & 0.29 \\
\hline $\mathrm{MnO}$ & 0.29 & 0.38 & 0.19 & 0.25 & 0.17 & 0.23 & 0.16 & 0.19 \\
\hline $\mathrm{Al}_{2} \mathrm{O}_{3}$ & 0.05 & 0.05 & 0.09 & 0.10 & 0.04 & 0.05 & 0.06 & 0.07 \\
\hline $\mathrm{TiO}_{2}$ & 0.02 & 0.03 & 0.03 & 0.01 & 0.01 & 0.03 & 0.00 & 0.01 \\
\hline $\mathrm{Cr}_{2} \mathrm{O}_{3}$ & 0.00 & 0.00 & 0.03 & 0.04 & 0.00 & 0.00 & 0.01 & 0.02 \\
\hline Total & 99.97 & 100.20 & 99.73 & 99.28 & 99.80 & 100.28 & 99.88 & 100.12 \\
\hline$\frac{\mathrm{Mg}}{\mathrm{Mg}+\mathrm{Fe}}$ & 0.838 & 0.813 & 0.835 & 0.833 & 0.866 & 0.852 & 0.865 & 0.863 \\
\hline
\end{tabular}

TABLE 10

Hole 444A Plagioclase Analyses

\begin{tabular}{|c|c|c|c|c|c|c|c|c|c|c|c|c|c|c|c|}
\hline \multirow{3}{*}{$\begin{array}{l}\text { Compo- } \\
\text { nent or } \\
\text { Ratio }\end{array}$} & \multicolumn{3}{|c|}{$20-1,27-29 \mathrm{~cm}$} & \multirow{3}{*}{$\begin{array}{c}\begin{array}{c}20-1, \\
62-64 \mathrm{~cm}\end{array} \\
\begin{array}{c}\text { Ground- } \\
\text { mass }\end{array} \\
1.0 \times \\
0.2 \mathrm{~mm}\end{array}$} & \multicolumn{3}{|c|}{$23-2,0-4 \mathrm{~cm}$} & \multicolumn{4}{|c|}{$26-1,49-54 \mathrm{~cm}$} & \multicolumn{4}{|c|}{$27-3,21-25 \mathrm{~cm}$} \\
\hline & \multirow{2}{*}{$\begin{array}{c}1.4 \times \\
0.2 \mathrm{~mm}\end{array}$} & \multirow{2}{*}{$\begin{array}{c}\text { Groundmass } \\
0.4 \times \\
0.2 \mathrm{~mm}\end{array}$} & \multirow{2}{*}{$\begin{array}{c}0.8 \times \\
0.2 \mathrm{~mm}\end{array}$} & & \multicolumn{2}{|c|}{$\begin{array}{l}\text { Phenocryst } \\
1.4 \times 0.8 \mathrm{~mm}\end{array}$} & \multirow{2}{*}{$\begin{array}{c}\text { Ground- } \\
\text { mass } \\
0.4 \times \\
0.1 \mathrm{~mm}\end{array}$} & \multicolumn{2}{|c|}{$\begin{array}{c}\text { Phenocryst } \\
1.75 \times 1.5 \mathrm{~mm}\end{array}$} & \multicolumn{2}{|c|}{$\begin{array}{l}\text { Groundmass } \\
0.5 \times 1.5 \mathrm{~mm}\end{array}$} & \multicolumn{2}{|c|}{$\begin{array}{c}\text { Phenocryst } \\
3.0 \times 1.8 \mathrm{~mm}\end{array}$} & \multicolumn{2}{|c|}{$\begin{array}{l}\text { Groundmass } \\
0.4 \times 0.1 \mathrm{~mm}\end{array}$} \\
\hline & & & & & core & rim & & core & $\mathrm{rim}$ & core & rim & core & rim & core & rim \\
\hline Mgo & 0.13 & 0.13 & 0.13 & 0.12 & 0.22 & 0.23 & 0.27 & 0.20 & 0.14 & 0.17 & 0.06 & .20 & .16 & .11 & .10 \\
\hline $\mathrm{FeO}$ & 0.55 & 0.48 & 0.50 & 0.63 & 0.34 & 0.56 & 0.68 & 0.34 & 0.70 & 0.75 & 0.58 & .41 & .63 & .85 & .69 \\
\hline $\mathrm{Na}_{2} \mathrm{O}$ & 3.64 & 3.75 & 2.86 & 5.01 & 2.03 & 3.63 & 3.88 & 1.77 & 4.62 & 4.78 & 7.15 & 2.01 & 4.39 & 4.03 & 6.01 \\
\hline $\mathrm{Al}_{2} \mathrm{O}_{3}$ & 30.64 & 30.62 & 32.10 & 28.92 & 32.87 & 30.34 & 30.04 & 33.82 & 29.96 & 29.17 & 25.59 & 32.23 & 29.15 & 29.93 & 27.20 \\
\hline $\mathrm{SiO}_{2}$ & 50.72 & 51.40 & 49.04 & 53.51 & 46.78 & 51.10 & 51.37 & 46.23 & 52.21 & 53.08 & 58.83 & 46.98 & 51.62 & 51.07 & 56.22 \\
\hline $\mathrm{K}_{2} \mathrm{O}$ & 0.26 & 0.27 & 0.19 & 0.38 & 0.03 & 0.07 & 0.08 & 0.02 & 0.10 & 0.10 & 0.20 & .03 & .09 & .07 & .15 \\
\hline $\mathrm{CaO}$ & 14.16 & 13.82 & 15.61 & 11.47 & 16.65 & 13.86 & 13.60 & 17.41 & 12.31 & 12.02 & 7.71 & 16.56 & 12.55 & 13.32 & 9.85 \\
\hline Total & 100.10 & 100.47 & 100.43 & 100.04 & 98.92 & 99.79 & 99.92 & 99.79 & 100.04 & 100.07 & 100.04 & 98.42 & 98.59 & 99.38 & 100.22 \\
\hline$\frac{\mathrm{Na}}{\mathrm{Na}+\mathrm{Ca}+\mathrm{K}}$ & 0.313 & 0.325 & 0.247 & 0.433 & 0.181 & 0.321 & 0.340 & 0.156 & 0.403 & 0.417 & 0.620 & 0.180 & 0.386 & 0.353 & 0.521 \\
\hline$\frac{\mathrm{Ca}}{\mathrm{Na}+\mathrm{Ca}+\mathrm{K}}$ & 0.672 & 0.660 & 0.742 & 0.546 & 0.817 & 0.675 & 0.656 & 0.843 & 0.591 & 0.578 & 0.369 & 0.818 & 0.609 & 0.643 & 0.471 \\
\hline
\end{tabular}


TABLE 11

Hole 444A K-Spar Analyses

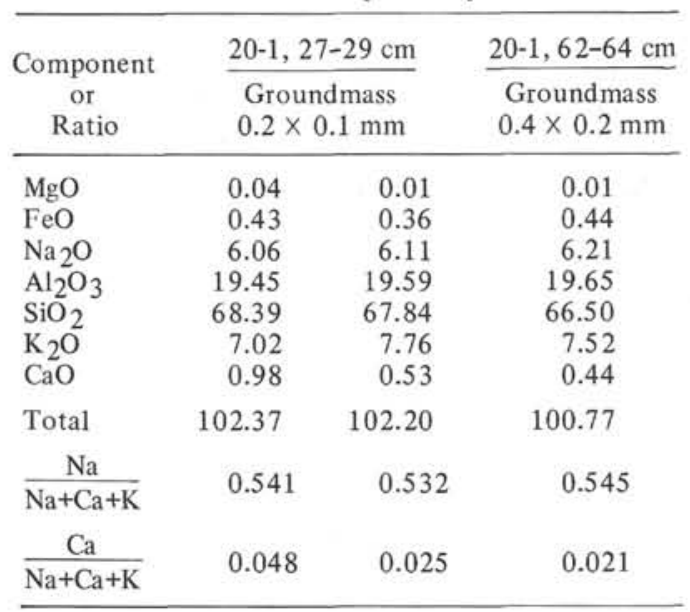

the overlying glassy chill zone, while this is not the case for olivines from the lower Site 444 sills. This suggests substantially more in situ fractionation of the melt in the Site 444 lower sills, which is consistent with the petrographic evidence.

\section{Pyroxene}

Pyroxenes in mid-ocean-ridge abyssal tholeiites are typically augite, or salite with compositions close to and grading into the augite field (e.g., Hodges and Papika, 1977; Mevel et al., 1978; Dungan et al., 1978). They are distinguished by a broad range of compositions trending roughly from augite towards ferrosilite, a characteristic typical of many basalts (e.g., Kuno, 1955). The pyroxenes in the Shikoku Basin abyssal tholeiites are augites with a fairly coherent composition trend parallel to that for Skaergaard augites (Figure 2), although compositions are far more limited.

While the range of pyroxene compositions is very limited in the Site 442 basalts (as is that for olivines), pyroxenes in the Site 443 and 444 basalts are more varied, consistent with the broader range of olivine compositions there. The rather coherent overall composition trend is somewhat atypical for abyssal basalts, possibly reflecting growth in relatively thick, slowly cooled units, which in many cases show evidence of in situ fractionation.

The pyroxenes from the Site 444 alkali olivine basalt are all salites and titanosalites, and in this respect are typical of alkali olivine basalts (e.g., Wilkinson, 1958). The composition trend of these pyroxenes (Fig. 2) is unusual. Rather than running parallel to the diopside-hedenbergite join with increasing iron, as is typical of many alkali olivine basalts (Wilkinson, 1958), they trend toward it. This trend essentially parallels that of the compositional differences between the different sectors in sector-zoned (hourglass) pyroxenes in these basalts (Fig. 2). Accordingly, we relate this trend to rapid cooling and rapid pyroxene growth, and to differences in the abilities of certain cations (notably magnesium, silicon, and titanium) to diffuse through the melt to the site of crystal growth.

The differences between the two groups of pyroxenes in the Shikoku Basin basalts are typical for tholeiites and alkali basalts (e.g., Wilkinson, 1958). These groups can also be distinguished on the basis of $\mathrm{Al}$ and $\mathrm{Si}$, or $\mathrm{Al}$ and weight per cent $\mathrm{TiO}_{2}$, as shown in Figure 4. Pyroxenes from the tholeiitic basalts in Figure 4 plot well within the range of the fields defined for tholeiites by LeBas (1962) and by Kushiro (1960). Those from the alkali basalts, however, extend from the alkaline field into

TABLE 12

Hole 444A Pyroxene Analyses

\begin{tabular}{|c|c|c|c|c|c|c|c|c|c|}
\hline \multirow{3}{*}{$\begin{array}{c}\text { Compo- } \\
\text { nent or } \\
\text { Ratio }\end{array}$} & \multirow{3}{*}{$\begin{array}{c}20-1, \\
27-29 \mathrm{~cm} \\
\begin{array}{c}\text { Pheno- } \\
\text { cryst } \\
1.75 \times \\
0.4 \mathrm{~mm}\end{array}\end{array}$} & \multirow{2}{*}{\multicolumn{2}{|c|}{$\begin{array}{c}20-1,27-59 \mathrm{~cm} \\
\\
\text { Groundmass } \\
0.5 \times 0.3 \mathrm{~mm} \\
\end{array}$}} & \multirow{3}{*}{$\begin{array}{c}20-1, \\
62-64 \mathrm{~cm} \\
\begin{array}{c}\text { Pheno- } \\
\text { cryst }\end{array} \\
1.75 \times \\
0.5 \mathrm{~mm}\end{array}$} & \multirow{2}{*}{\multicolumn{2}{|c|}{$\begin{array}{c}20-1,62-64 \mathrm{~cm} \\
\text { Groundmass } \\
0.4 \times 0.25 \mathrm{~mm} \\
\end{array}$}} & \multirow{3}{*}{$\begin{array}{c}\begin{array}{c}23-2, \\
0-4 \mathrm{~cm}\end{array} \\
\begin{array}{c}\text { Pheno- } \\
\text { cryst }\end{array} \\
1.75 \times \\
1.0 \mathrm{~mm}\end{array}$} & \multirow{3}{*}{$\begin{array}{c}26-1, \\
\frac{49-54 \mathrm{~cm}}{\text { Ground- }} \\
\text { mass } \\
0.4 \times \\
0.3 \mathrm{~mm}\end{array}$} & \multirow{3}{*}{$\begin{array}{c}27-3, \\
21-25 \mathrm{~cm} \\
\text { Ground- } \\
\text { mass } \\
0.2 \times \\
0.2 \mathrm{~mm}\end{array}$} \\
\hline & & & & & & & & & \\
\hline & & Sector A & Sector B & & Sector A & Sector B & & & \\
\hline Mgo & 14.10 & 15.53 & 13.49 & 10.94 & 11.97 & 10.21 & 18.14 & 16.12 & 14.59 \\
\hline $\mathrm{FeO}$ & 7.28 & 6.05 & 7.05 & 10.29 & 10.64 & 11.24 & 4.32 & 7.06 & 10.19 \\
\hline $\mathrm{Na}_{2} \mathrm{O}$ & 0.43 & 0.36 & 0.48 & 0.57 & 0.46 & 0.56 & 0.32 & 0.26 & 0.44 \\
\hline $\mathrm{Al}_{2} \mathrm{O}_{3}$ & 4.03 & 2.59 & 5.71 & 7.29 & 4.58 & 7.89 & 3.36 & 2.17 & 2.45 \\
\hline $\mathrm{SiO}_{2}$ & 49.82 & 52.14 & 48.57 & 45.66 & 49.03 & 44.53 & 52.53 & 52.75 & 50.86 \\
\hline $\mathrm{K}_{2} \mathrm{O}$ & 0.02 & 0.03 & 0.04 & 0.01 & 0.04 & 0.03 & 0.02 & 0.00 & 0.00 \\
\hline $\mathrm{CaO}$ & 22.09 & 22.73 & 22.63 & 21.74 & 22.49 & 21.90 & 19.60 & 20.51 & 18.94 \\
\hline $\mathrm{TiO}_{2}$ & 1.81 & 1.04 & 2.22 & 3.59 & 1.64 & 3.89 & 0.29 & 0.73 & 1.37 \\
\hline $\mathrm{Cr}_{2} \mathrm{O}_{3}$ & 0.12 & 0.22 & 0.27 & 0.08 & 0.07 & 0.06 & 0.56 & 0.10 & 0.00 \\
\hline $\mathrm{MnO}$ & 0.23 & 0.21 & 0.19 & 0.32 & 0.23 & 0.26 & 0.09 & 0.16 & 0.44 \\
\hline Total & 99.93 & 100.90 & 100.65 & 100.49 & 100.75 & 100.57 & 99.23 & 99.86 & 99.28 \\
\hline$\frac{\mathrm{Mg}}{\mathrm{Mg}+\mathrm{Fe}}$ & 0.776 & 0.821 & 0.774 & 0.655 & 0.667 & 0.619 & 0.882 & 0.803 & 0.719 \\
\hline$\frac{\mathrm{Mg}}{\mathrm{Mg}+\mathrm{Fe}+\mathrm{Ca}}$ & 0.414 & 0.441 & 0.401 & 0.339 & 0.351 & 0.317 & 0.524 & 0.463 & 0.430 \\
\hline$\frac{\mathrm{Ca}}{\mathrm{Mg}+\mathrm{Fe}+\mathrm{Ca}}$ & 0.466 & 0.463 & 0.482 & 0.483 & 0.473 & 0.488 & 0.406 & 0.423 & 0.401 \\
\hline
\end{tabular}


TABLE 13

Hole 444A Olivine Analyses

\begin{tabular}{|c|c|c|c|c|}
\hline \multirow{3}{*}{$\begin{array}{c}\text { Component } \\
\text { or Ratio }\end{array}$} & \multirow{3}{*}{$\begin{array}{l}\frac{20-1,27-29 \mathrm{~cm}}{\text { Groundmass }} \\
0.2 \times 0.2 \mathrm{~mm}\end{array}$} & \multirow{3}{*}{$\frac{26-1,49-54 \mathrm{~cm}}{\text { Groundmass }}$} & \multicolumn{2}{|c|}{$27-3,21-25 \mathrm{~cm}$} \\
\hline & & & \multicolumn{2}{|c|}{ Groundmass } \\
\hline & & & $0.25 \times 0.25 \mathrm{~mm}$ & $0.15 \times 0.2 \mathrm{~mm}$ \\
\hline $\mathrm{MgO}$ & 46.26 & 39.17 & 36.74 & 35.17 \\
\hline $\mathrm{FeO}$ & 14.18 & 22.51 & 25.64 & 27.34 \\
\hline $\mathrm{SiO}_{2}$ & 39.00 & 38.17 & 37.64 & 37.56 \\
\hline $\mathrm{CaO}^{2}$ & 0.23 & 0.28 & 0.25 & 0.25 \\
\hline $\mathrm{MnO}$ & 0.25 & 0.51 & 0.55 & 0.66 \\
\hline $\mathrm{Al}_{2} \mathrm{O}_{3}$ & 0.06 & 0.07 & 0.05 & 0.05 \\
\hline $\mathrm{TiO}_{2}$ & 0.00 & 0.00 & 0.04 & 0.05 \\
\hline $\mathrm{Cr}_{2} \mathrm{O}_{3}$ & 0.00 & 0.03 & 0.04 & 0.04 \\
\hline Total & 99.98 & 100.74 & 100.95 & 101.12 \\
\hline \multicolumn{5}{|l|}{$\mathrm{Mg}$} \\
\hline$\overline{\mathrm{Mg}+\mathrm{Fe}}$ & 0.853 & 0.756 & 0.719 & 0.697 \\
\hline
\end{tabular}

TABLE 14

Hole 444A Potassium-Bearing Analcite

\begin{tabular}{|c|c|c|c|c|c|c|c|}
\hline $\begin{array}{l}\text { Com- } \\
\text { ponent }\end{array}$ & $\begin{array}{c}1 \\
20-1, \\
71-73 \mathrm{~cm} \\
\text { Area } 3\end{array}$ & $\begin{array}{c}2 \\
20-1, \\
27-29 \mathrm{~cm} \\
\text { Area } 5\end{array}$ & $\begin{array}{c}3 \\
20-1 . \\
27-29 \mathrm{~cm} \\
\text { Area } 7\end{array}$ & $\begin{array}{c}4 \\
20-1, \\
27-29 \mathrm{~cm} \\
\text { Area } 4\end{array}$ & $\begin{array}{c}5 \\
20-1, \\
27-29 \mathrm{~cm} \\
\text { Area } 1 \\
\text { (M6) }\end{array}$ & $\begin{array}{c}6^{\mathrm{a}} \\
20-1, \\
27-29 \mathrm{~cm} \\
\text { Area } 1 \\
\text { (M7) }\end{array}$ & $\begin{array}{c}7 \\
20-1, \\
27-29 \mathrm{~cm} \\
\text { Area } 6\end{array}$ \\
\hline $\begin{array}{l}\mathrm{MgO} \\
\mathrm{FeO} \mathrm{Na}_{2} \mathrm{O} \\
\mathrm{Al}_{2} \mathrm{O}_{3} \\
\mathrm{SiO}_{2} \\
\mathrm{~K}_{2} \mathrm{O} \\
\mathrm{CaO}^{2} \mathrm{TiO}_{2} \\
\mathrm{H}_{2} \mathrm{Ob}^{2}\end{array}$ & $\begin{array}{r}0.04 \\
0.02 \\
1.54 \\
28.14 \\
57.69 \\
4.66 \\
2.20 \\
0.00 \\
5.71\end{array}$ & $\begin{array}{r}0.09 \\
0.02 \\
3.58 \\
28.07 \\
52.41 \\
5.13 \\
3.29 \\
0.00 \\
7.41\end{array}$ & $\begin{array}{r}0.13 \\
0.09 \\
4.39 \\
26.15 \\
52.60 \\
5.31 \\
2.00 \\
0.06 \\
9.27\end{array}$ & $\begin{array}{r}0.14 \\
0.05 \\
4.58 \\
27.79 \\
50.01 \\
4.87 \\
3.34 \\
0.00 \\
9.22\end{array}$ & $\begin{array}{r}0.11 \\
0.07 \\
5.25 \\
26.01 \\
52.77 \\
5.24 \\
2.06 \\
0.00 \\
8.49\end{array}$ & $\begin{array}{r}0.08 \\
0.07 \\
9.05 \\
24.51 \\
52.56 \\
4.86 \\
1.10 \\
0.00 \\
7.77\end{array}$ & $\begin{array}{r}0.11 \\
0.09 \\
6.60 \\
24.85 \\
51.96 \\
8.33 \\
1.20 \\
0.04 \\
6.82\end{array}$ \\
\hline Total & 100.00 & 100.00 & 100.00 & 100.00 & 100.00 & 100.00 & 100.00 \\
\hline $\begin{array}{l}\mathrm{Mg} \\
\mathrm{Fe} \\
\mathrm{Na} \\
\mathrm{NI} \\
\mathrm{Si} \\
\mathrm{K} \\
\mathrm{Ca} \\
\mathrm{Ti} \\
\mathrm{OH} \\
\mathrm{OH}\end{array}$ & $\begin{array}{l}0.002 \\
0.000 \\
0.110 \\
1.215 \\
2.108 \\
0.218 \\
0.086 \\
0.000 \\
1.395\end{array}$ & $\begin{array}{l}0.004 \\
0.000 \\
0.257 \\
1.220 \\
1.930 \\
0.242 \\
0.131 \\
0.000 \\
1.826\end{array}$ & $\begin{array}{l}0.007 \\
0.002 \\
0.310 \\
1.121 \\
1.907 \\
0.247 \\
0.079 \\
0.002 \\
2.248\end{array}$ & $\begin{array}{l}0.007 \\
0.002 \\
0.325 \\
1.198 \\
1.825 \\
0.226 \\
0.130 \\
0.000 \\
2.249\end{array}$ & $\begin{array}{l}0.007 \\
0.002 \\
0.375 \\
1.125 \\
1.932 \\
0.245 \\
0.082 \\
0.0000 \\
2.077\end{array}$ & $\begin{array}{l}0.004 \\
0.002 \\
0.654 \\
1.076 \\
1.954 \\
0.231 \\
0.045 \\
0.000 \\
1.933\end{array}$ & $\begin{array}{l}0.007 \\
0.002 \\
0.487 \\
1.114 \\
1.973 \\
0.405 \\
0.048 \\
0.002 \\
1.731\end{array}$ \\
\hline $\begin{array}{l}\mathrm{Ca}+\mathrm{Mg} \\
+\mathrm{Na}+\mathrm{K}\end{array}$ & 0.416 & 0.634 & 0.643 & 0.688 & 0.709 & 0.934 & 0.947 \\
\hline
\end{tabular}

"Matches analysis of potassium-bearing analcite phenocrysts in basalt, Highwood Mis., Montana, cited by Deer, Howic, and Zussman (1963), p. 343.
$\mathrm{H}_{2} \mathrm{O}=100-\Sigma$ Oxides.

the peralkaline field, possibly reflecting a late peralkaline mesostasis.

We note that all these characteristics are also typical of pyroxenes in the alkaline and tholeiitic basalts drilled at Site 446 in the Daito Basin during Leg 58 (see Dick, in prep.).

\section{Feldspar}

Plagioclase microphenocrysts in the Site 442 and 443 pillow-basalt glasses are fairly calcic $\left(\mathrm{An}_{88}-\mathrm{An}_{65}\right)$, with slightly more sodic rims (Figure 2). In some cases, the rims are reversely zoned, with a small decrease in soda. On the other hand, the plagioclase in the sills from all three sites is strongly zoned, with rim compositions $\left(\mathrm{An}_{72}-\mathrm{An}_{38}\right)$ far more sodic than core compositions $\left(A n_{77}-A n_{58}\right)$. No reversely zoned plagioclases in the sills were analyzed, although a wide range of compositions is common in many thin sections. The plagioclase in the Site 444 sills tends to be more sodic than at the other Shikoku Basin sites. Also seen in the Site 444 plagioclase compositions is a trend of increasing $\mathrm{K}_{2} \mathrm{O}$ with increasing sodium, the alkaline sill containing late interstitial anorthoclase. This trend is not clearly seen in the remaining Shikoku Basin Leg 48 basalts.

\section{Other Minerals}

Kaersutite, analcite, biotite, chlorite, and zeolites were also analyzed from the Site 444 alkali basalt. The zeolites appear to include phillipsite and natrolite. The analcites are curious, with anomalously high $\mathrm{K}_{2} \mathrm{O}$ contents. Analysis No. 6 in Table 14 matches the most-potassic analcite cited by Deer, Howie, and Zussman (1963); most of the analyses would appear to be of some other mineral were there not a nearly continuous gradation among them, with a wide range of soda contents. The titaniferous hornblende, kaersutite, is very similar to that in the alkali-basalt sills in the Daito Basin at Site 446.

\section{CHEMISTRY}

\section{Analytical Methods}

Major-element analyses of whole-rock powders of the non-glassy interiors of pillow lavas and massive cooling units were done by X-ray fluorescence at the University of Birmingham. Representative analyses and averages for the principal Shikoku Basin units are shown in Tables 17 to 24 ; the analytical details and the complete set of analyses can be found in Marsh et al. (this volume). Electron-microprobe analyses of natural basalt glasses were made both by W. G. Melson (Smith-

TABLE 15

Hole 444A Zeolite Analyses

\begin{tabular}{|c|c|c|c|c|c|c|}
\hline & \multirow{2}{*}{$\frac{20-1,22-27 \mathrm{~cm}}{1.75 \times 1.0 \mathrm{~mm}}$} & \multicolumn{2}{|c|}{$20-1,62-64 \mathrm{~cm}$} & \multicolumn{2}{|c|}{$20-1,71-73 \mathrm{~cm}$} & \multirow{2}{*}{$\frac{20-3,30-32 \mathrm{~cm}}{0.4 \times 0.2 \mathrm{~mm}}$} \\
\hline & & $0.6 \times 0.3 \mathrm{~mm}$ & $0.4 \times 0.3 \mathrm{~mm}$ & $0.4 \times 0.2 \mathrm{~mm}$ & $1.75 \times 0.8 \mathrm{~mm}$ & \\
\hline $\mathrm{MgO}$ & 0.00 & 0.05 & 0.10 & 0.07 & 0.09 & 0.06 \\
\hline $\mathrm{FeO}$ & 0.03 & 0.11 & 0.09 & 0.02 & 0.04 & 0.13 \\
\hline $\mathrm{Na}_{2} \mathrm{O}$ & 7.17 & 12.18 & 6.09 & 7.12 & 6.10 & 3.45 \\
\hline $\mathrm{Al}_{2} \mathrm{O}_{3}$ & 26.61 & 19.94 & 23.42 & 23.61 & 21.74 & 23.27 \\
\hline $\mathrm{SiO}_{2}$ & 47.98 & 41.22 & 45.77 & 45.73 & 48.47 & 51.09 \\
\hline $\mathrm{K}_{2} \mathrm{O}$ & 3.38 & 6.91 & 5.74 & 4.74 & 3.83 & 4.36 \\
\hline $\mathrm{CaO}$ & 2.73 & 0.76 & 2.23 & 1.95 & 0.65 & 1.68 \\
\hline $\mathrm{TiO}_{2}$ & 0.00 & 0.00 & 0.01 & 0.00 & 0.00 & 0.07 \\
\hline $\mathrm{H}_{2} \mathrm{O}^{\mathrm{a}}$ & 12.10 & 18.83 & 15.65 & 16.76 & 19.08 & 15.89 \\
\hline Total & 100.00 & 100.00 & 100.00 & 100.00 & 100.00 & 100.00 \\
\hline
\end{tabular}

\footnotetext{
${ }^{\mathrm{a}} \mathrm{H}_{2} \mathrm{O}=100-\Sigma$ oxides.
} 
TABLE 16

Hole 444A Kaersutite, Biotite, and Chlorite Analyses

\begin{tabular}{|c|c|c|c|c|}
\hline & \multirow{3}{*}{$\begin{array}{c}\frac{20-1,62-64 \mathrm{~cm}}{\text { Kaersutite }} \\
0.9 \times 0.4 \mathrm{~mm}\end{array}$} & \multirow{3}{*}{$\frac{19, \mathrm{CC}}{\text { Biotite }}$} & \multicolumn{2}{|c|}{$20-1,62-64 \mathrm{~cm}$} \\
\hline & & & \multicolumn{2}{|c|}{ Chlorite } \\
\hline & & & $0.4 \times 0.1 \mathrm{~mm}$ & $0.4 \times 0.1 \mathrm{~mm}$ \\
\hline $\mathrm{MgO}$ & 11.04 & 15.75 & 16.03 & 15.69 \\
\hline $\mathrm{FeO}$ & 14.35 & 14.42 & 25.73 & 25.44 \\
\hline $\mathrm{Na}_{2} \mathrm{O}$ & 2.76 & 0.85 & 0.52 & 1.49 \\
\hline $\mathrm{Al}_{2} \mathrm{O}_{3}$ & 11.93 & 10.49 & 14.38 & 14.34 \\
\hline $\mathrm{SiO}_{2}$ & 39.71 & 39.13 & 33.37 & 32.90 \\
\hline $\mathrm{K}_{2} \mathrm{O}$ & 0.92 & 7.25 & 0.18 & 0.20 \\
\hline $\mathrm{CaO}$ & 11.87 & 0.04 & 0.18 & 0.13 \\
\hline $\mathrm{TiO}_{2}$ & 6.26 & 3.35 & 0.21 & 0.15 \\
\hline $\mathrm{Cr}_{2} \mathrm{O}_{3}$ & 0.01 & 0.02 & 0.04 & 0.44 \\
\hline $\mathrm{MnO}$ & 0.19 & 0.17 & 0.41 & 0.34 \\
\hline $\mathrm{H}_{2} \mathrm{O}^{\mathrm{a}}$ & 0.96 & 8.53 & 8.95 & 8.88 \\
\hline Total & 100.00 & 100.00 & 100.00 & 100.00 \\
\hline
\end{tabular}

${ }^{\mathrm{a}} \mathrm{H}_{2} \mathrm{O}=100-\Sigma$ oxides.

sonian Institution) and by us (Massachusetts Institute of Technology) on the same samples. Analytical procedures are the same as those described by Melson et al. (1976). The glass analyses from the two laboratories were not significantly different; for purposes of comparison to the large data set already available from the Smithsonian, their data are given in Table 25.

\section{Results}

The majority of the Leg 58 Shikoku Basin basalts contain normative olivine and hypersthene and are classed as tholeiitic basalts. On the AFM diagram (Figure 5), they show a strong iron-enrichment trend similar to that of other abyssal-tholeiite suites. As seen in Table 25, the average analyses of the Shikoku Basin tholeiites (excluding the upper sill of Hole 444A), although a little low in iron, are surprisingly close to the average of abyssal spreading-center basalt glasses found by Melson et al. (1976). A few XRF whole-rock analyses from Site 442 contain normative nepheline; however, none of the Hole 442B basalt glasses contains nepheline, and we suspect that the normative nepheline is due to the effects of alteration on the crystalline interiors of the flows. The petrography and chemistry indicate that, while the glasses are essentially unaltered, the relatively crystalline basalts are often extensively altered. For example, while only one of the basalt glasses contains more than 0.24 weight per cent $\mathrm{K}_{2} \mathrm{O}$, the $\mathrm{XRF}$ analysis of the tholeiites averages $0.25 \pm 0.12$ weight per cent $\mathrm{K}_{2} \mathrm{O}$. As expected from its mineralogy, the upper sill at Site 444 is an alkali olivine basalt, containing abundant normative orthoclase and plotting well within the alkaline field on an alkalis-versus-silica plot (Figure 6).

The basalt analyses for the Shikoku Basin give consistently low totals. Because both microprobe analyses of natural glass and XRF analyses of whole-rock powders have low totals, and because these totals are similar for most units (Tables 17 to 24), we feel that this is a real feature, not due to analytical error. The most likely explanation is a primary volatile content higher than that of most abyssal basalts.

On the basis of normative-olivine content, the basalts can be divided into tholeiites ( $\leq 5 \%$ normative olivine) and olivine tholeiites ( $\geq 5 \%$ normative olivine). As seen in Figure 7 , the average whole-rock analysis has a normative-olivine content intermediate between the tholeiite and olivine-tholeiite basalt glasses. We feel that this is largely the result of silica loss during alteration not affecting the glassy chill zones and accumulation of olivine crystals in many samples, particularly the massive portions of compound pillow-lava flows. Although the pillow lavas are overlain by most of the sills at Site 442 , they are not as primitive, containing substantially less normative olivine.

In the normative plagioclase-olivine-pyroxene ternary (Figure 8), the Shikoku Basin basalts plot along a trend which resembles the experimentally determined 1-atm plagioclase-olivine and plagioclase-pyroxene cotectics in the system An-Di-Fo. Although this trend differs in detail from the experimental cotectics, this is undoubtedly due to the greater complexity of the natural system. The colinearity of the Shikoku Basin basalts, however, does indicate that crystallization of these basalts was closely controlled by the phase boundaries (see also Miyashiro et al., 1970). The position and nature of these boundaries change significantly with pressure (e.g., O'Hara, 1968; Presnall et al., 1978), and the present colinearity of both "differentiated"- and "primitive"-appearing basalts indicates that none of them could represent an unmodified "primary" mantle liquid.

As with basalts from the Cayman Trough and those from $24^{\circ} \mathrm{N}$ on the Mid-Atlantic Ridge (Thompson et al., in press; Dick and Bryan, 1978), the trend of the Shiko$\mathrm{ku}$ Basin basalts is offset slightly into the plagioclase field compared to trends for other mid-ocean-ridge basalts. While the Cayman Trough basalts are richer in soda than other mid-ocean-ridge basalts and have higher titania contents at a given value of $\mathrm{FeO} / \mathrm{MgO}$ (Thompson et al., in press), this is not generally the case for the Shikoku Basin tholeiites. In fact, although the soda content is similar in most cases to other abyssal tholeiites, they generally have lower titania contents at a given value of $\mathrm{FeO} / \mathrm{MgO}$ (Figure 9). The position of the cotectic curve in the $\mathrm{Px}-\mathrm{Pg}-\mathrm{Ol}$ normative ternary is not fixed, but varies with pressure and the bulk composition of the liquids. For example, greater or lesser amounts of water, soda, lime, and silica all could shift the cotectic curve laterally into or away from the plagioclase field. Although one can genetically relate the composition of groups of basalts lying along the same cotectic trend in the ternary, this is not the case for basalts lying along different cotectic trends. In this case, the different compositions must represent variations in the source region, either in mantle composition or in the conditions of melting, such as the amount of water available during fusion.

Further differences between most abyssal basalt suites and the Shikoku Basin tholeiites can be seen using an $\mathrm{MgO}-\mathrm{FeO}-\mathrm{Al}_{2} \mathrm{O}_{3}$ discriminant plot (Figure 10). Pearce et al. (1971) has successfully used this plot to discriminate between basalts from five separate tectonic environments, as shown in Figure 10. The boundary between ocean-ridge and floor (abyssal) and continental 


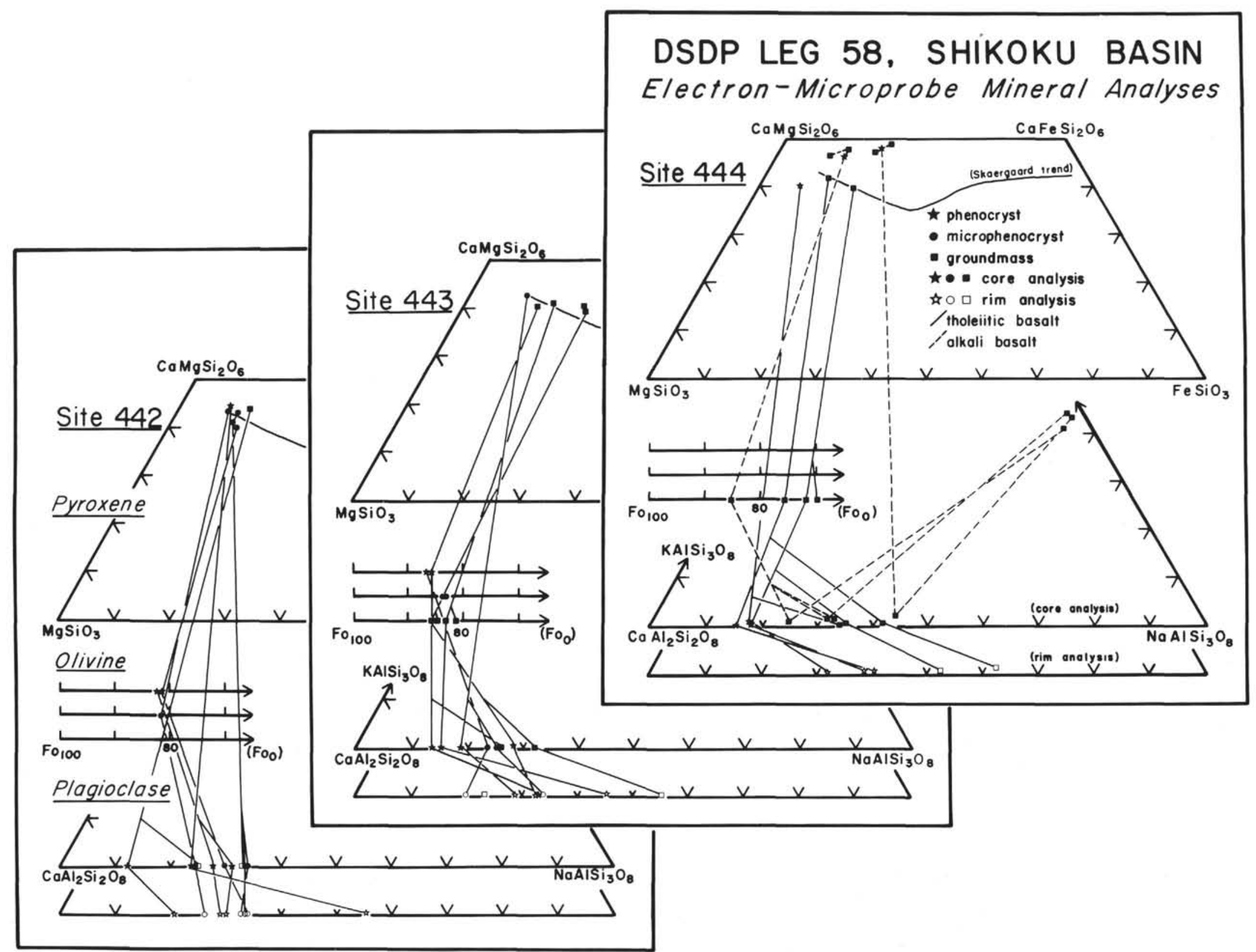
section. Dashed lines indicate alkali basalts; solid lines indicate tholeites. 


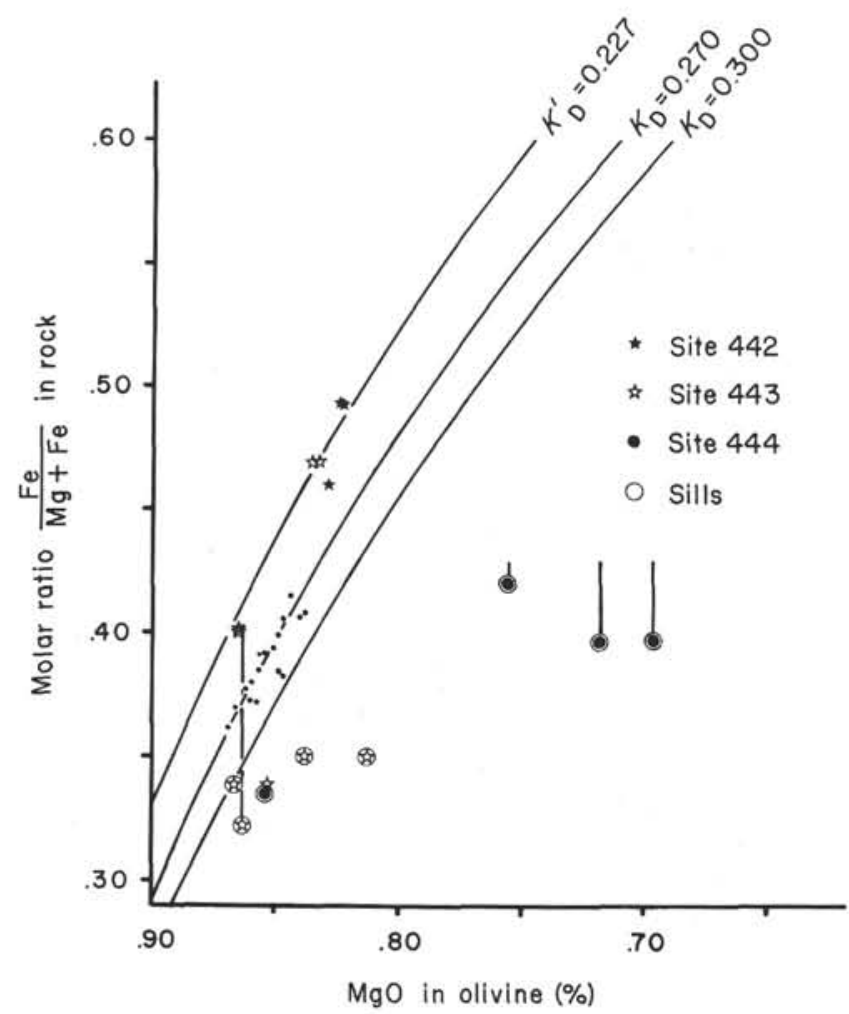

Figure 3. Forsterite content of olivine versus the molar ratio $\mathrm{Fe} /(\mathrm{Mg}+\mathrm{Fe})$ of coexisting glass from pillowbasalt rims, and XRF whole-rock analyses of Site 443 and 444 sills. Lines connect the points representing whole-rock and coexisting olivine compositions in a sill to the composition of the glass in the chilled margin. Small dots are compositions of olivine-glass pairs for basalts from Hole 396B, near the MidAtlantic Ridge (from Dungan et al., 1978). Lines plotted in diagram are for various $\mathrm{Fe}-\mathrm{Mg}$ distribution coefficients.

basalts was found to be a particularly effective discriminant, excluding all but one of the 96 tested analyses. Overall, the abyssal field proved to be a 76 per cent effective discriminant. While Pearce et al. used only analyses with greater than 51 weight per cent $\mathrm{SiO}_{2}$, they feel that the diagram remains an effective discriminant for abyssal basalts with less silica (Pearce, pers. comm.). The Site 442B basalts, however, plot across the boundaries in the abyssal, continental, and orogenic fields. The Site 443 basalts are more effectively discriminated (some analyses only slightly into the continental field), while nearly all the Hole 444A basalts lie within the abyssal field.

Taken separately, in Figure 10, the pillow lavas lie outside the abyssal field at Site 442 and are transitional at Site 443. On the other hand, the younger sills (or massive cooling units), by themselves, are a typical abyssal suite. While the sills are clearly off-axis intrusives, this is not clearly evident for the pillow basalts. In addition, it is evident from Figures 5 to 9 that the pillow basalts are generally further evolved than the sills, and the two could not be comagmatic.
The alkaline-basalt sill from Hole 444A appears to be similar in most respects to the many small, differentiated alkaline sills, such as the Black Jack Sill of New South Wales (Wilkinson, 1958) or the analcite-dolerite sheets of Atumi, Japan (Kushiro, 1964). According to the classification of Irvine and Baragar (1971), the sill is an iron-rich alkali olivine basalt, also similar to rocks from Tristan $\mathrm{Da}$ Cunha, Nightengale, and Crough islands in the Atlantic. Petrographically and normatively, the sill could be reasonably called a teschenite, and a description of these rocks given by Wilkinson (1968) closely matches the Hole 444A sill. Although Ca-poor pyroxene was not found, the linear variation of the Hole 444A upper-sill analyses across the AFM diagram (Figure 7) is nearly identical to that of the Atumi sill. Accordingly, the sill appears to have affinities to the basalt-trachyandesite-trachyte series (see Kuno, 1968, pp. 679-680). The alkaline sill is also very similar to the alkaline basalts found at Site 446 in the Daito Basin, which are also intercalated with tholeiitic sills. This indicates that a back-arc-basin provenance is plausible for the Site 446 sills.

The transitional character seen in the chemistry and mineralogy of many of the Leg 58 Shikoku Basin basalts can also be seen in their trace-element composition. Shown in Table 25 are averages of the XRF analyses for selected trace elements for each of the Shikoku Basin sites. The complete analyses and a detailed interpretation of these data can be found in Marsh et al. (this volume). As seen from the table, the Shikoku Basin basalts are very similar to Marianas back-arc-basin basalts; they differ from typical mid-ocean-ridge tholeiites in having typically lower concentrations of $\mathrm{Ni}$ and $\mathrm{Cr}$ and greater amounts of $\mathrm{Sr}$ and $\mathrm{Ba}$. These are characteristics also shared by island-arc tholeiites and alkaline rocks, and in this respect the Shikoku Basin basalts are transitional from abyssal tholeiite to these two rock types. With regards to alkaline rocks, however, the titania content even of the alkali basalt from Site 444 is lower than typical, and despite their elevated $\mathrm{Sr}$ and $\mathrm{Ba}$ contents, the tholeiites do not appear to have elevated $\mathrm{NaO}+\mathrm{K}_{2} \mathrm{O}$ at a given concentration of $\mathrm{SiO}_{2}$. In addition, the Shikoku Basin basalts have much higher concentrations of $\mathrm{Cr}, \mathrm{Ni}, \mathrm{Ti}$, and $\mathrm{Zr}$ than islandarc tholeiites. Thus, while they do have a clearly transitional character, they appear to be distinct both from typical alkaline and are basalts with respect to trace elements.

\section{Stratigraphic Variation}

In Figure 11, the compositions of Shikoku Basin basalts are plotted against their estimated depth in the hole, based on expanded recoveries. In addition to providing a visualization of intra- and inter-unit variations, these plots allow us to compare the data from glass and whole-rock analyses, for which there are striking differences within a unit for many elements. Only in the case of titania is agreement generally excellent. The differences between the two data sets can be reasonably attributed to one of four factors: (1) systematic errors in analysis by one or the other technique, (2) the effects of 

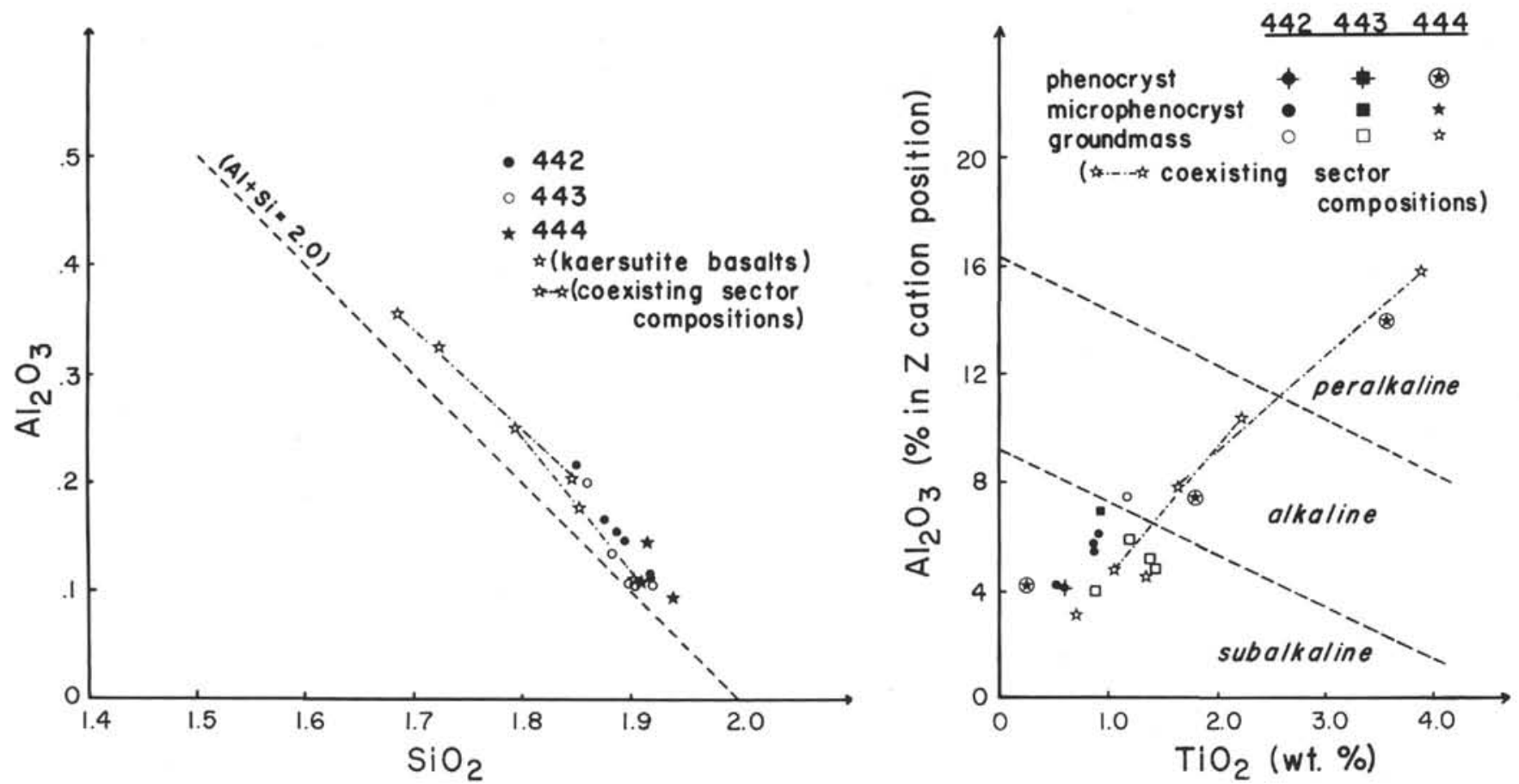

Figure 4. Molar Al versus Si and versus weight per cent $\mathrm{TiO}_{2}$ for pyroxenes from Leg 58 Shikoku Basin basalts. Fields for pyroxenes from LeBas (1962) and Kushiro (1960).

TABLE 17

Representative XRF Whole-Rock Analyses and CIPW Norms

\begin{tabular}{|c|c|c|c|c|c|c|c|c|c|c|c|}
\hline \multirow[b]{2}{*}{$\begin{array}{c}\text { Compo- } \\
\text { nent }\end{array}$} & \multicolumn{4}{|c|}{ Hole 442B } & \multicolumn{5}{|c|}{ Hole 443} & \multicolumn{2}{|c|}{ Hole 444A } \\
\hline & $\begin{array}{c}6-2, \\
32-34 \mathrm{~cm} \\
1 \mathrm{H}\end{array}$ & $\begin{array}{c}13-3 \\
19-21 \mathrm{~cm} \\
2 \mathrm{~A}\end{array}$ & $\begin{array}{c}16-1, \\
47-49 \mathrm{~cm} \\
2 \mathrm{~B}\end{array}$ & $\begin{array}{c}20-1 \\
50-52 \mathrm{~cm} \\
2 \mathrm{~B}\end{array}$ & $\begin{array}{c}50-4 \\
74-77 \mathrm{~cm} \\
2 \mathrm{~A}\end{array}$ & $\begin{array}{c}52-2, \\
12-14 \mathrm{~cm} \\
3 \mathrm{~A}\end{array}$ & $\begin{array}{c}57-2, \\
81-83 \mathrm{~cm} \\
4 \mathrm{C}\end{array}$ & $\begin{array}{c}59-3, \\
47-49 \mathrm{~cm} \\
5 \mathrm{C}\end{array}$ & $\begin{array}{c}60-2, \\
10-12 \mathrm{~cm} \\
5 \mathrm{D}\end{array}$ & $\begin{array}{c}20-2 \\
5-7 \mathrm{~cm} \\
1\end{array}$ & $\begin{array}{c}24-2, \\
73-75 \mathrm{~cm} \\
2 \mathrm{~A}\end{array}$ \\
\hline $\mathrm{SiO}_{2}$ & 49.80 & 49.50 & 49.70 & 50.10 & 49.90 & 49.90 & 49.90 & 49.70 & 49.10 & 47.20 & 50.00 \\
\hline $\mathrm{Al}_{2} \mathrm{O}_{3}$ & 15.60 & 16.10 & 16.60 & 15.90 & 16.40 & 16.30 & 15.20 & 16.30 & 15.80 & 16.40 & 16.00 \\
\hline $\mathrm{FeO}$ & 8.14 & 9.71 & 8.85 & 9.86 & 9.76 & 9.15 & 9.32 & 8.22 & 8.68 & 7.64 & 9.29 \\
\hline $\mathrm{MgO}$ & 6.97 & 5.20 & 5.51 & 5.47 & 6.30 & 7.11 & 6.73 & 7.95 & 8.57 & 7.70 & 7.25 \\
\hline $\mathrm{CaO}$ & 12.88 & 12.27 & 12.67 & 12.04 & 11.58 & 12.11 & 12.20 & 11.62 & 11.07 & 8.43 & 11.09 \\
\hline $\mathrm{Na}_{2} \mathrm{O}$ & 2.73 & 2.79 & 2.39 & 2.76 & 2.79 & 2.65 & 2.68 & 2.71 & 2.52 & 3.62 & 2.72 \\
\hline $\mathrm{K}_{2} \mathrm{O}$ & 0.26 & 0.40 & 0.38 & 0.47 & 0.20 & 0.07 & 0.39 & 0.10 & 0.09 & 1.90 & 0.22 \\
\hline $\mathrm{MnO}$ & 0.13 & 0.14 & 0.17 & 0.17 & 0.17 & 0.16 & 0.14 & 0.13 & 0.16 & 0.16 & 0.17 \\
\hline $\mathrm{TiO}_{2}$ & 1.16 & 1.58 & 1.23 & 1.62 & 1.56 & 1.36 & 1.69 & 1.22 & 1.24 & 1.41 & 1.59 \\
\hline $\mathrm{P}_{2} \mathrm{O}_{5}$ & 0.15 & 0.35 & 0.22 & 0.22 & 0.18 & 0.14 & 0.17 & 0.11 & 0.12 & 0.34 & 0.22 \\
\hline Total & 97.8 & 97.7 & 97.7 & 98.6 & 98.8 & 99.0 & 98.4 & 98.1 & 97.4 & 94.8 & 98.6 \\
\hline Or & 1.54 & 2.36 & 2.25 & 2.78 & 1.18 & 0.41 & 2.30 & 0.59 & 0.53 & 11.23 & 1.30 \\
\hline $\mathrm{Ab}$ & 23.10 & 23.61 & 20.22 & 23.36 & 23.61 & 22.42 & 22.68 & 22.93 & 21.32 & 19.94 & 23.02 \\
\hline An & 29.55 & 30.23 & 33.45 & 29.61 & 31.64 & 32.38 & 28.29 & 32.02 & 31.54 & 22.89 & 30.80 \\
\hline $\mathrm{Ne}$ & - & - & - & - & - & - & - & - & - & - & - \\
\hline $\mathrm{Di}$ & 27.37 & 23.64 & 23.19 & 23.85 & 20.39 & 22.04 & 25.63 & 20.35 & 18.45 & 13.62 & 18.73 \\
\hline $\mathrm{Hy}$ & 4.22 & 8.04 & 13.02 & .58 & 11.38 & 10.77 & 8.18 & 9.97 & 13.07 & - & 14.56 \\
\hline OI & 9.50 & 6.38 & 2.74 & 5.86 & 7.27 & 8.03 & 7.73 & 9.63 & 9.80 & 17.87 & 6.61 \\
\hline II & 2.20 & 3.00 & 2.34 & 3.08 & 2.96 & 2.58 & 3.21 & 2.32 & 2.36 & 2.68 & 3.02 \\
\hline Ap & 0.35 & 0.81 & 0.51 & 0.51 & 0.42 & 0.32 & 0.39 & 0.25 & 0.28 & 0.79 & 0.51 \\
\hline
\end{tabular}

hydrothermal alteration at elevated temperatures, (3) the effects of deep sea "weathering," or (4) differences in glass and whole-rock compositions due to the presence of crystals, particularly phenocrysts.

Errors in analysis should produce systematic differences for each element analyzed by the two techniques. Such systematic differences are not immediately recognizable on careful inspection of Figure 11. Rather, the differences fall into one of three cases; the XRF values for a given element are (1) equal to or greater than the microprobe value $\left(\mathrm{K}_{2} \mathrm{O}, \mathrm{P}_{2} \mathrm{O}_{5}, \mathrm{CaO}\right)$, (2) equal to or less than the microprobe value $\left(\mathrm{SiO}_{2}, \mathrm{FeO}, \mathrm{NaO}\right),(3)$ or vary randomly or systematically within a given unit about the microprobe value $\left(\mathrm{TiO}_{2}, \mathrm{Al}_{2} \mathrm{O}_{3}, \mathrm{MgO}\right)$. In fact, examination of analyses of closely spaced glass and whole-rock samples reveals that in these cases agree- 
TABLE 18

Hole 442B Basalt Glass Analyses and CIPW Norms

\begin{tabular}{|c|c|c|c|c|c|c|c|c|c|c|c|c|c|c|c|c|}
\hline $\begin{array}{c}\text { Compo- } \\
\text { nent }\end{array}$ & $\begin{array}{c}6-2, \\
42-44 \mathrm{~cm}\end{array}$ & $\begin{array}{c}10-1 \\
86-88 \mathrm{~cm}\end{array}$ & $\begin{array}{c}10-2, \\
16-19 \mathrm{~cm}\end{array}$ & $\begin{array}{c}11-1, \\
13-15 \mathrm{~cm}\end{array}$ & $\begin{array}{c}11-3, \\
6-10 \mathrm{~cm}\end{array}$ & $\begin{array}{c}12-1, \\
7-10 \mathrm{~cm}\end{array}$ & $\begin{array}{c}13-3 \\
11-14 \mathrm{~cm}\end{array}$ & $\begin{array}{c}14-1, \\
41-45 \mathrm{~cm}\end{array}$ & $\begin{array}{c}15-1 \\
16-18 \mathrm{~cm}\end{array}$ & $\begin{array}{l}17-1, \\
0-5 \mathrm{~cm}\end{array}$ & $\begin{array}{c}17-1 \\
16-18 \mathrm{~cm}\end{array}$ & $\begin{array}{c}17-2, \\
11-16 \mathrm{~cm}\end{array}$ & $\begin{array}{r}18-1 \\
7-9 \mathrm{~cm}\end{array}$ & $\begin{array}{c}19-1, \\
7-13 \mathrm{~cm}\end{array}$ & $\begin{array}{c}19-2, \\
65-70 \mathrm{~cm}\end{array}$ & $\begin{array}{c}20-1, \\
57-61 \mathrm{~cm}\end{array}$ \\
\hline $\mathrm{SiO}_{2}$ & 49.78 & 49.49 & 50.05 & 50.55 & 50.78 & 49.96 & 50.80 & 50.62 & 50.78 & 50.62 & 51.09 & 51.43 & 51.42 & 50.96 & 51.16 & 51.33 \\
\hline $\mathrm{Al}_{2} \mathrm{O}_{3}$ & 16.18 & 14.97 & 17.54 & 15.69 & 15.80 & 15.56 & 15.82 & 15.90 & 15.69 & 15.58 & 15.13 & 15.06 & 14.99 & 14.93 & 14.91 & 15.15 \\
\hline $\mathrm{FeO}^{\mathrm{a}}$ & 8.76 & 10.10 & 6.87 & 9.64 & 9.67 & 9.60 & 9.59 & 9.66 & 9.64 & 9.56 & 10.30 & 10.24 & 10.27 & 10.34 & 10.20 & 10.29 \\
\hline $\mathrm{MgO}$ & 7.00 & 6.18 & 6.78 & 6.23 & 6.43 & 6.26 & 6.29 & 6.36 & 6.34 & 5.76 & 5.95 & 6.10 & 5.86 & 5.81 & 5.92 & 5.97 \\
\hline $\mathrm{CaO}$ & 11.85 & 11.16 & 14.05 & 10.92 & 11.05 & 10.81 & 10.99 & 11.04 & 10.94 & 11.11 & 10.80 & 10.81 & 10.78 & 10.75 & 10.77 & 10.76 \\
\hline $\mathrm{Na}_{2} \mathrm{O}$ & 2.61 & 2.90 & 2.57 & 2.89 & 2.94 & 2.89 & 2.93 & 2.92 & 2.91 & 2.63 & 3.04 & 3.01 & 3.04 & 3.06 & 3.05 & 3.02 \\
\hline $\mathrm{K}_{2} \mathrm{O}$ & 0.15 & 0.12 & 0.10 & 0.18 & 0.17 & 0.16 & 0.18 & 0.18 & 0.18 & 0.26 & 0.20 & 0.21 & 0.21 & 0.20 & 0.19 & 0.22 \\
\hline $\mathrm{MnO}$ & 0.17 & 0.21 & 0.20 & 0.20 & 0.18 & 0.17 & 0.23 & 0.20 & 0.21 & 0.17 & 0.21 & 0.19 & 0.21 & 0.15 & 0.18 & 0.18 \\
\hline $\mathrm{TiO}_{2}$ & 1.19 & 1.57 & 1.15 & 1.54 & 1.56 & 1.53 & 1.50 & 1.52 & 1.48 & 1.30 & 1.62 & 1.60 & 1.64 & 1.58 & 1.59 & 1.65 \\
\hline $\mathrm{P}_{2} \mathrm{O}_{5}$ & 0.12 & 0.16 & 0.10 & 0.17 & 0.18 & 0.17 & 0.17 & 0.16 & 0.18 & 0.16 & 0.16 & 0.18 & 0.19 & 0.17 & 0.16 & 0.19 \\
\hline Total & 97.81 & 96.83 & 99.41 & 98.01 & 98.76 & 97.11 & 98.50 & 98.56 & 98.35 & 97.15 & 98.50 & 98.83 & 98.61 & 97.95 & 98.13 & 98.76 \\
\hline \multicolumn{17}{|l|}{ Q } \\
\hline $\begin{array}{l}\mathrm{Or} \\
\mathrm{Ab}\end{array}$ & $\begin{array}{r}0.89 \\
22.09\end{array}$ & $\begin{array}{r}0.71 \\
24.54\end{array}$ & $\begin{array}{r}0.59 \\
21.75\end{array}$ & $\begin{array}{r}1.06 \\
24.46\end{array}$ & $\begin{array}{r}1.00 \\
24.88\end{array}$ & $\begin{array}{r}0.95 \\
24.46\end{array}$ & $\begin{array}{r}1.06 \\
24.79\end{array}$ & $\overline{1}$ & $\overline{1}$ & $\begin{array}{r}0.43 \\
1.54\end{array}$ & $-\overline{18}$ & $\overline{124}$ & - & - & 12 & $\overline{1.30}$ \\
\hline An & 31.99 & 27.48 & 36.03 & 29.31 & 29.41 & 29.01 & $\begin{array}{l}24.19 \\
29.48\end{array}$ & $\begin{array}{r}1.06 \\
24.71\end{array}$ & $\begin{array}{r}1.06 \\
24.62\end{array}$ & $\begin{array}{r}1.54 \\
22.26\end{array}$ & $\begin{array}{r}1.18 \\
25.72\end{array}$ & $\begin{array}{r}1.24 \\
25.47\end{array}$ & $\begin{array}{l}1.24 \\
25.72\end{array}$ & $\begin{array}{r}1.18 \\
25.89\end{array}$ & $\begin{array}{l}1.12 \\
25.81\end{array}$ & 25.56 \\
\hline $\mathrm{Di}$ & 21.39 & 22.26 & 26.95 & 19.66 & 20.03 & 19.44 & 19.79 & 29.75 & 29.22 & 29.94 & 27.05 & 26.96 & 26.64 & 26.41 & 26.43 & 27.13 \\
\hline $\mathrm{Hy}$ & 12.26 & 12.46 & 3.24 & 17.13 & 15.70 & 15.98 & 16.50 & 19.83 & 19.75 & 20.03 & 21.17 & 21.15 & 21.28 & 21.46 & 21.55 & 20.77 \\
\hline OI & 6.65 & 6.07 & 8.43 & 3.07 & 4.35 & 3.98 & 3.63 & 15.30 & 17.14 & 20.12 & 17.13 & 18.72 & 19.13 & 17.25 & 18.01 & 18.70 \\
\hline II & 2.26 & 2.98 & 2.18 & 2.92 & 2.96 & 2.91 & 2.85 & 4.65 & 3.33 & - & 2.79 & 1.83 & 1.05 & 2.36 & 1.81 & 1.74 \\
\hline \multirow{2}{*}{ Ap } & 0.28 & 0.37 & 0.23 & 0.39 & 0.42 & 0.39 & 0.39 & 2.89 & 2.81 & 2.47 & 3.08 & 3.04 & 3.11 & 3.00 & 3.02 & 3.13 \\
\hline & & & & & & & & 0.37 & 0.42 & 0.37 & 0.37 & 0.42 & 0.44 & 0.39 & 0.37 & 0.44 \\
\hline
\end{tabular}

${ }^{a}$ Total iron as $\mathrm{FeO}$. Norms calculated using all iron as $\mathrm{FeO}$.

TABLE 19

Hole 443 Basalt Glass Analyses and CIPW Norms

\begin{tabular}{|c|c|c|c|c|c|c|c|c|c|c|c|c|c|c|c|c|}
\hline $\begin{array}{c}\text { Compo- } \\
\text { nent }\end{array}$ & $\begin{array}{c}49-3, \\
59-62 \mathrm{~cm}\end{array}$ & $\begin{array}{c}54-8 \\
43-48 \mathrm{~cm}\end{array}$ & $\begin{array}{c}55-1, \\
28-33 \mathrm{~cm}\end{array}$ & $\begin{array}{c}55-2, \\
125-130 \mathrm{~cm}\end{array}$ & $\begin{array}{c}56-1 \\
10-13 \mathrm{~cm}\end{array}$ & $\begin{array}{c}57-1, \\
71-75 \mathrm{~cm}\end{array}$ & $\begin{array}{c}57-2, \\
100-107 \mathrm{~cm}\end{array}$ & $\begin{array}{c}57-3 \\
1-16 \mathrm{~cm}\end{array}$ & $\begin{array}{c}58-4 \\
135-141 \mathrm{~cm}\end{array}$ & $\begin{array}{c}58-5 \\
1-10 \mathrm{~cm}\end{array}$ & $\begin{array}{c}59-2, \\
63-67 \mathrm{~cm}\end{array}$ & $\begin{array}{c}59-3 \\
77-81 \mathrm{~cm}\end{array}$ & $\begin{array}{c}61-3, \\
70-73 \mathrm{~cm}\end{array}$ & $\begin{array}{c}62-2, \\
141-145 \mathrm{~cm}\end{array}$ & $\begin{array}{c}62-3, \\
121-125 \mathrm{~cm}\end{array}$ & $\begin{array}{r}64-1, \\
1-7 \mathrm{~cm}\end{array}$ \\
\hline $\mathrm{SiO}_{2}$ & 50.02 & 50.73 & 50.63 & 50.78 & 50.34 & 50.54 & 50.51 & 51.07 & 50.21 & 50.01 & 50.13 & 49.73 & 49.67 & 50.34 & 50.13 & 50.15 \\
\hline $\mathrm{Al}_{2} \mathrm{O}_{3}$ & 15.90 & 15.55 & 15.69 & 15.53 & 15.73 & 15.39 & 15.36 & 15.77 & 16.46 & 16.22 & 16.38 & 16.38 & 16.42 & 16.09 & 16.28 & 16.26 \\
\hline $\mathrm{FeO}^{\mathrm{a}}$ & 8.88 & 10.17 & 9.68 & 10.29 & 10.22 & 9.39 & 10.46 & 9.97 & 9.15 & 8.97 & 8.93 & 8.95 & 8.94 & 9.10 & 8.91 & 8.96 \\
\hline $\mathrm{MgO}$ & 5.33 & 6.39 & 6.47 & 6.72 & 6.35 & 6.41 & 6.61 & 6.35 & 7.77 & 7.81 & 7.84 & 7.84 & 7.84 & 7.25 & 7.46 & 7.49 \\
\hline $\mathrm{CaO}$ & 10.62 & 10.88 & 10.86 & 10.94 & 10.83 & 10.89 & 10.68 & 10.82 & 11.84 & 11.80 & 11.92 & 11.75 & 11.94 & 11.92 & 11.74 & 11.78 \\
\hline $\mathrm{Na}_{2} \mathrm{O}$ & 3.28 & 2.78 & 2.87 & 2.71 & 2.66 & 2.67 & 2.81 & 2.91 & 2.86 & 2.80 & 2.83 & 2.85 & 2.78 & 2.85 & 2.79 & 2.69 \\
\hline $\mathrm{K}_{2} \mathrm{O}$ & 0.56 & 0.23 & 0.23 & 0.23 & 0.24 & 0.24 & 0.22 & 0.23 & 0.10 & 0.11 & 0.09 & 0.10 & 0.10 & 0.15 & 0.17 & 0.16 \\
\hline Mno & 0.21 & 0.19 & 0.20 & 0.19 & 0.21 & 0.21 & 0.23 & 0.22 & 0.13 & 0.15 & 0.14 & 0.16 & 0.15 & 0.16 & 0.16 & 0.18 \\
\hline $\mathrm{TiO}_{2}$ & 1.79 & 1.65 & 1.60 & 1.69 & 1.67 & 1.59 & 1.76 & 1.68 & 1.31 & 1.34 & 1.34 & 1.30 & 1.27 & 1.40 & 1.33 & 1.32 \\
\hline $\mathrm{P}_{2} \mathrm{O}_{5}^{\prime}$ & 0.17 & 0.20 & 0.18 & 0.19 & 0.17 & 0.18 & 0.15 & 0.19 & 0.13 & 0.10 & 0.11 & 0.12 & 0.13 & 0.12 & 0.12 & 0.13 \\
\hline Total & 96.76 & 98.77 & 98.41 & 99.27 & 98.42 & 97.51 & 98.79 & 99.21 & 99.96 & 99.31 & 99.71 & 99.18 & 99.24 & 99.38 & 99.09 & 99.12 \\
\hline Or & 3.31 & 1.36 & 1.36 & 1.36 & 1.42 & 1.42 & 1.30 & 1.36 & 0.59 & 0.65 & 0.53 & 0.59 & 0.59 & 0.89 & 1.00 & 0.95 \\
\hline $\mathrm{Ab}$ & 27.76 & 23.52 & 24.29 & 22.93 & 22.51 & 22.59 & 23.78 & 24.62 & 24.20 & 23.69 & 23.95 & 24.12 & 23.52 & 24.12 & 23.61 & 22.76 \\
\hline An & 27.01 & 29.27 & 29.25 & 29.53 & 30.27 & 29.30 & 28.65 & 29.29 & 31.78 & 31.37 & 31.73 & 31.61 & 32.03 & 30.67 & 31.40 & 31.82 \\
\hline $\mathrm{Di}$ & 20.35 & 19.38 & 19.38 & 19.44 & 18.51 & 19.45 & 19.34 & 19.16 & 21.42 & 21.74 & 21.87 & 21.23 & 21.60 & 22.75 & 21.39 & 21.15 \\
\hline Hy & 7.87 & 18.93 & 16.92 & 18.99 & 19.65 & 21.20 & 17.65 & 18.03 & 6.81 & 7.56 & 6.84 & 5.81 & 5.86 & 8.32 & 8.93 & 10.78 \\
\hline $\mathrm{OI}$ & 6.67 & 2.71 & 3.76 & 3.37 & 2.49 & 0.12 & 4.39 & 3.12 & 12.38 & 11.53 & 11.99 & 13.08 & 12.92 & 9.71 & 9.96 & 8.85 \\
\hline II & 3.40 & 3.13 & 3.04 & 3.21 & 3.17 & 3.02 & 3.34 & 3.19 & 2.49 & 2.54 & 2.54 & 2.47 & 2.41 & 2.66 & 2.53 & 2.51 \\
\hline Ap & 0.39 & 0.46 & 0.42 & 0.44 & 0.39 & 0.42 & 0.35 & 0.44 & 0.30 & 0.23 & 0.25 & 0.28 & 0.30 & 0.28 & 0.28 & 0.30 \\
\hline
\end{tabular}

${ }^{\mathrm{a}} \mathrm{Total}$ iron as $\mathrm{FeO}$. 
TABLE 20

\begin{tabular}{|c|c|c|}
\hline Component & $\begin{array}{c}23-2 \\
0-4 \mathrm{~cm}\end{array}$ & $\begin{array}{c}26-1 \text {, } \\
9-11 \mathrm{~cm}\end{array}$ \\
\hline $\mathrm{SiO}_{2}$ & 51.18 & 51.32 \\
\hline $\mathrm{Al}_{2} \mathrm{O}_{3}$ & 14.86 & 15.61 \\
\hline $\mathrm{FeO}^{\mathrm{a}}$ & 10.53 & 8.69 \\
\hline $\mathrm{MgO}$ & 6.79 & 6.49 \\
\hline $\mathrm{CaO}$ & 11.58 & 12.13 \\
\hline $\mathrm{Na}_{2} \mathrm{O}$ & 3.42 & 3.41 \\
\hline $\mathrm{K}_{2} \mathrm{O}$ & 0.21 & 0.22 \\
\hline $\mathrm{MnO}$ & 0.19 & 0.19 \\
\hline $\mathrm{TiO}_{2}$ & 1.78 & 1.54 \\
\hline $\mathrm{P}_{2} \mathrm{O}_{5}$ & 0.25 & 0.25 \\
\hline Total & 100.79 & 99.85 \\
\hline Or & 1.24 & 1.30 \\
\hline $\mathrm{Ab}$ & 28.94 & 28.86 \\
\hline An & 24.58 & 26.64 \\
\hline Di & 25.84 & 26.26 \\
\hline Hy & 3.30 & 3.10 \\
\hline oi & 12.93 & 10.19 \\
\hline 1 & 3.38 & 2.92 \\
\hline $4 p$ & 0.58 & 0.58 \\
\hline
\end{tabular}

${ }^{\mathrm{a}}$ Total iron as $\mathrm{FeO}$.

TABLE 21

Average Analyses for Site 442 Basalt Lithologic Units ${ }^{\mathrm{a}}$

\begin{tabular}{|c|c|c|c|c|c|c|}
\hline \multirow[b]{2}{*}{ Component } & \multicolumn{2}{|c|}{ Unit 1 (Sills) } & \multicolumn{2}{|c|}{ Unit 2 (Pillows) } & \multicolumn{2}{|c|}{ Unit 3 (Pillows) } \\
\hline & Rock $^{\mathrm{b}}$ & Glass $^{c}$ & Rock $^{b}$ & Glass ${ }^{c}$ & Rock $^{b}$ & Glass $\mathrm{c}$ \\
\hline $\mathrm{SiO}_{2}$ & 49.63 & 49.78 & 49.50 & 50.38 & 50.04 & 51.14 \\
\hline $\mathrm{Al}_{2} \mathrm{O}_{3}$ & 15.76 & 16.18 & 16.18 & 15.87 & 16.24 & 15.11 \\
\hline $\mathrm{FeO}^{\mathrm{d}}$ & 8.10 & 8.76 & 9.92 & 9.35 & 9.08 & 10.17 \\
\hline $\mathrm{MgO}$ & 7.23 & 7.00 & 5.17 & 6.36 & 5.71 & 5.91 \\
\hline $\mathrm{CaO}$ & 12.05 & 11.85 & 12.20 & 11.37 & 12.17 & 10.83 \\
\hline $\mathrm{Na}_{2} \mathrm{O}$ & 2.79 & 2.61 & 2.76 & 2.87 & 2.74 & 2.98 \\
\hline $\mathrm{K}_{2} \mathrm{O}$ & 0.28 & 0.15 & 0.38 & 0.16 & 0.38 & 0.21 \\
\hline $\mathrm{MnO}$ & 0.13 & 0.17 & 0.17 & 0.20 & 0.16 & 0.18 \\
\hline $\mathrm{TiO}_{2}$ & 1.20 & 1.19 & 1.62 & 1.48 & 1.42 & 1.57 \\
\hline $\mathrm{P}_{2} \mathrm{O}_{5}$ & 0.15 & 0.12 & 0.28 & 0.16 & 0.22 & 0.17 \\
\hline Total & 97.32 & 97.81 & 98.18 & 98.21 & 98.16 & 98.27 \\
\hline $\begin{array}{l}\text { (Number } \\
\text { of samples) }\end{array}$ & (34) & (1) & (13) & (7) & (21) & (15) \\
\hline
\end{tabular}

${ }^{\text {a All Site }} 442$ rocks are aphyric ( $<1 \%$ phenocrysts).

Whole-rock XRF analysis of the interior of a cooling unit or pillow.

${ }_{\mathrm{d}}^{\mathrm{c}}$ Electron-microprobe analysis of glass chip from a sill zone.

$\mathrm{d}_{\text {Total iron as } \mathrm{FeO} \text {. }}$

ment is often excellent for nearly all elements. These factors suggest that the differences in the XRF and microprobe data sets are not generally due to differences in analytical technique.

The principal difference between the two data sets is the general uniformity of the glass analyses within specific units and sub-units, which contrasts sharply to the often large variations of the whole-rock analyses. This is to be anticipated. First, the glasses represent the liquid composition at the time of eruption and, unlike the whole rock, will not show the effects of phenocryst accumulation or in situ fractional crystallization. Second, while alteration is clearly evident in the more-crystalline samples selected for XRF analysis, the glasses selected
TABLE 22

Average Analyses for Site 443 Basalt Lithologic Units

\begin{tabular}{lrrrr}
\hline & Unit $^{\mathrm{a}}$ & \multicolumn{2}{c}{ Phyric Sills $^{\mathrm{b}}$} & \multicolumn{2}{c}{ Aphyric Basalts } \\
Component & Glass & \multicolumn{1}{c}{ Rock } & Rock & Glass \\
\hline $\mathrm{SiO}_{2}{ }^{\mathrm{d}}$ & 50.02 & 49.47 & 49.59 & 50.33 \\
$\mathrm{Al}_{2} \mathrm{O}_{3}$ & 15.90 & 16.10 & 15.49 & 15.97 \\
$\mathrm{FeO}^{\mathrm{e}}$ & 8.88 & 8.78 & 8.79 & 9.47 \\
$\mathrm{MgO}$ & 5.33 & 7.65 & 7.71 & 7.11 \\
$\mathrm{CaO}$ & 10.62 & 11.71 & 11.69 & 11.37 \\
$\mathrm{Na}_{2} \mathrm{O}$ & 3.28 & 2.65 & 2.66 & 2.79 \\
$\mathrm{~K}_{2} \mathrm{O}$ & 0.56 & 0.14 & 0.25 & 0.17 \\
$\mathrm{MnO}_{\mathrm{TiO}}$ & 0.21 & 0.17 & 0.14 & 0.18 \\
$\mathrm{P}_{2} \mathrm{O}_{5}$ & 1.79 & 1.34 & 1.43 & 1.48 \\
$\mathrm{Total}$ & 0.17 & 0.14 & 0.14 & 0.15 \\
& 96.76 & 98.15 & 97.89 & 99.02 \\
(Number & & & & \\
of samples) & $(1)$ & $(30)$ & $(36)$ & $(15)$ \\
\hline
\end{tabular}

${ }^{\text {a }}$ Pyroxene-plagioclase glassy basalt.

Plagioclase-olivine phyric sills, units 2, 4, 5C, 6A, 6B.

${ }^{c}$ Aphyric pillows and flows (all extrusive?), units $3,5 \mathrm{~A}$, $5 \mathrm{~B}, 5 \mathrm{D}, 5 \mathrm{E}$

$\mathrm{d}_{\text {Average } \mathrm{SiO}_{2} \text { content }}=49.67 \%$.

$\mathrm{e}_{\text {Total iron as } \mathrm{FeO} \text {. }}$

TABLE 23

Average Analyses for Site 444 Basalt Lithologic Units

\begin{tabular}{|c|c|c|c|}
\hline \multirow[b]{2}{*}{ Component } & \multirow{2}{*}{$\begin{array}{l}\text { Unit } 1 \\
\text { Rock }\end{array}$} & \multicolumn{2}{|c|}{ Unit 2} \\
\hline & & Rock & Glass \\
\hline $\mathrm{SiO}_{2}$ & 47.80 & 49.70 & 51.25 \\
\hline $\mathrm{Al}_{2} \mathrm{O}_{3}$ & 16.26 & 16.27 & 15.24 \\
\hline $\mathrm{FeO}^{\mathrm{a}}$ & 7.54 & 8.71 & 9.61 \\
\hline $\mathrm{MgO}$ & 7.64 & 7.11 & 6.64 \\
\hline $\mathrm{CaO}$ & 8.39 & 11.72 & 11.86 \\
\hline $\mathrm{Na}_{2} \mathrm{O}$ & 3.60 & 2.68 & 3.42 \\
\hline $\mathrm{K}_{2} \mathrm{O}$ & 1.97 & 0.22 & 0.22 \\
\hline $\mathrm{MnO}$ & 0.15 & 0.18 & 0.19 \\
\hline $\mathrm{TiO}_{2}$ & 1.55 & 1.44 & 1.66 \\
\hline $\mathrm{P}_{2} \mathrm{O}_{5}$ & 0.32 & 0.21 & 0.25 \\
\hline Total & 95.22 & 98.24 & 100.34 \\
\hline $\begin{array}{l}\text { (Number } \\
\text { of samples) }\end{array}$ & (5) & (10) & (2) \\
\hline
\end{tabular}

${ }^{\mathrm{a}} \mathrm{Total}$ iron as $\mathrm{FeO}$.

for microprobe analysis are generally fresh and show little sign of alteration. Because it is difficult to separate the effects of the other processes on the whole-rock analyses, interpretation of the igneous petrogenesis from major-element data of these rocks should be based as much as possible on the glass analyses.

Generally speaking, if the XRF analysis of an element is uniformly lower or higher than the glass analysis in a unit-particularly in an aphyric basalt-one would expect that this difference is the product of either hydrothermal alteration or "weathering". Thus, it would appear for example that $\mathrm{SiO}_{2}$ has been leached out of the Hole $442 \mathrm{~B}$ pillow lavas, while $\mathrm{CaO}, \mathrm{K}_{2} \mathrm{O}$ and $\mathrm{P}_{2} \mathrm{O}_{5}$ appear to have increased. This would be of critical importance to our interpretation of the XRF analyses in the 
TABLE 24

Regional Average Analyses

\begin{tabular}{|c|c|c|c|c|c|c|}
\hline \multirow{2}{*}{$\begin{array}{l}\text { Com- } \\
\text { ponent }\end{array}$} & \multirow{2}{*}{$\begin{array}{c}\text { Leg }^{\mathrm{a}} \\
58 \\
\text { Avg. }\end{array}$} & \multicolumn{2}{|c|}{ MORB Glass ${ }^{b}$} & \multirow{2}{*}{$\begin{array}{c}\text { Mid- } \\
\text { A tlantic } \\
\text { Ridgec } \\
\text { (glass) }\end{array}$} & \multirow{2}{*}{$\begin{array}{c}\text { East } \\
\text { Pacific } \\
\text { Rise }^{c}\end{array}$} & \multirow{2}{*}{$\begin{array}{c}\text { Arc } \\
\text { Tholeiite }^{\mathrm{d}}\end{array}$} \\
\hline & & Avg. & $\sigma$ & & & \\
\hline $\mathrm{SiO}_{2}$ & 49.87 & 50.54 & 1.78 & 50.68 & 50.19 & 49.0 \\
\hline $\mathrm{Al}_{2} \mathrm{O}_{3}$ & 15.84 & 15.27 & 1.13 & 15.60 & 14.86 & 18.6 \\
\hline $\mathrm{FeO}^{\mathrm{e}}$ & 8.97 & 10.69 & 1.67 & 9.85 & 11.33 & 9.6 \\
\hline $\mathrm{MgO}$ & 6.90 & 7.29 & 1.06 & 7.69 & 7.10 & 5.1 \\
\hline $\mathrm{CaO}$ & 11.74 & 11.16 & 1.54 & 11.44 & 11.44 & 9.0 \\
\hline $\mathrm{Na}_{2} \mathrm{O}$ & 2.76 & 2.69 & 0.77 & 2.66 & 2.66 & 3.0 \\
\hline $\mathrm{K}_{2} \mathrm{O}$ & 0.25 & 0.22 & 0.23 & 0.17 & 0.16 & 0.58 \\
\hline $\mathrm{MnO}$ & 0.16 & - & - & - & - & - \\
\hline $\mathrm{TiO}_{2}$ & 1.41 & 1.62 & 0.50 & 1.49 & 1.77 & 0.8 \\
\hline $\mathrm{P}_{2} \mathrm{O}_{5}$ & 0.17 & 0.14 & 0.06 & 0.12 & 0.14 & 0.14 \\
\hline Total & 98.07 & 99.61 & & & & 99.62 \\
\hline
\end{tabular}

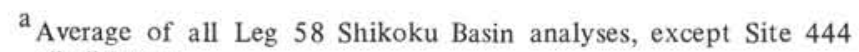
alkali basalt.

Average of all data from Melson et al. (1976).

${ }^{c}$ Average from Melson et al. (1976) for Mid-Atlantic Ridge and East Pacific Rise.

${ }^{d}$ Average of seven island-arc tholeiites from Hawkins (1977).

Total iron as FeO.

discriminant diagram of Pearce et. al. (1971), were it not confirmed by the plot of the fresh-glass data as well. Other variations within units or sub-units can be reasonably attributed to differences in whole-rock and glass compositions arising from in situ crystal-liquid fractionation, or flow differentiation, particularly where there is a significant variation in titania. This may be the case for magnesium in the Hole 442B pillow lavas, where a systematic upward increase in modal feldspar could explain the differences between the two data sets. Overall, in the absence of glass analyses, all variations in whole-rock compositions should be judged critically, with the exception of titanium, and to a lesser degree magnesium and phosphorus.

The Leg 58 basalts can be divided into a number of chemically distinct units at each of the Shikoku Basin drill sites: four at Site 442, seven at Site 443, and three at Site 444 . These units are best defined by abrupt changes in alteration-resistant $\mathrm{TiO}_{2}$ and $\mathrm{P}_{2} \mathrm{O}_{5}$, and their boundaries coincide in most cases with abrupt changes in magnetic inclination and (or) lithologic-unit or sub- unit boundaries as defined by the shipboard scientific party. A sharp break in the chemistry within unit 2B at Site 443 suggests that it is actually at least two separate cooling units and that there should be an intervening lithologic boundary.

The uppermost unit at Site 442, the uppermost two units at Site 443 (excluding the minor unit 1), and all units at Site 444 consist entirely of massive cooling units, with no intervening pillow lavas. From the general presence of fine-grained rather than glassy chill zones (and other evidence previously discussed), these appear to be sill sequences (including sub-sediment flows as sills).

The lower geochemical units at Sites 442 and 443, however, contain both massive cooling units and pillow lavas within individual geochemical units. Many adjoining pillow lavas and massive cooling units also share the same magnetic inclinations. In some cases, the massive cooling unit may be the lower portion of a compound pillow flow (i.e., bottom of unit 2 and middle of unit 3 at Site 442 and unit 3 at Site 443). In other cases, as indicated by the lack of adjoining pillow lavas and the presence of thick, glassy chill zones, the cooling units must be consanguineous extrusive sheet flows or shallow sills (sub-sediment flows) intercalated with the pillow lavas (i.e., unit 5 and sub-units $3 \mathrm{E}$ to $3 \mathrm{I}$ at Site 443).

While the composition of the initial magma (as defined by the composition of the chill zones) is uniform among the sub-units within each geochemical unit, there are often substantial variations within individual massive sills and flows which may exceed the overall chemical variability between the initial compositions of the different units at the site. These variations are generally sympathetic: increases in silica and titania generally are matched by decreases in magnesia. Such variations are consistent with in situ fractional crystallization (particularly of olivine) and differentiation of the cooling units (note particularly unit $1 \mathrm{~J}$ at Site 442 ; units $4,5 \mathrm{D}$, and $6 \mathrm{~A}$ at Site 443; and units 1 and $2 \mathrm{~B}$ at Site 444).

Although there are considerable differences in composition among units, there does not appear to be any systematic stratigraphic variation; rather, the upward chemical variation of geochemical units in the Shikoku Basin seems to be of the "random-walk" variety. The coincidence of changes in magnetic inclination with ei-

TABLE 25

Trace-Element Data for the Shikoku Basin, and Comparative Analyses ${ }^{\text {a }}$

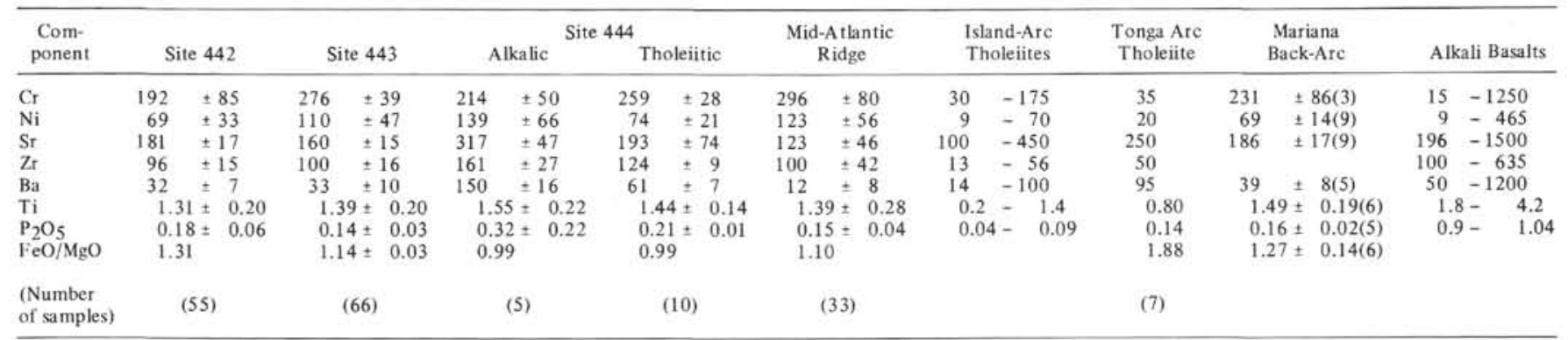

${ }^{\mathrm{a}}$ Shikoku Basin data are averages from the complete XRF data set given by Marsh et al. (this volume); all data are quoted in ppm except TiO ${ }_{2}$ and $\mathrm{P}_{2} \mathrm{O}_{5}$, which are in weight per cent; average for Mid-A tlantic Ridge tholeites is from Melson and Thompson (1971); Tonga arc tholeite average from Hawkins (1977); ranges for Tonga and Hakone are tholeiites from Miyashiro (1978); alkali-basalt ranges are also from Miyashiro (1978); FeO represents total iron. $\pm=1 \sigma$. 


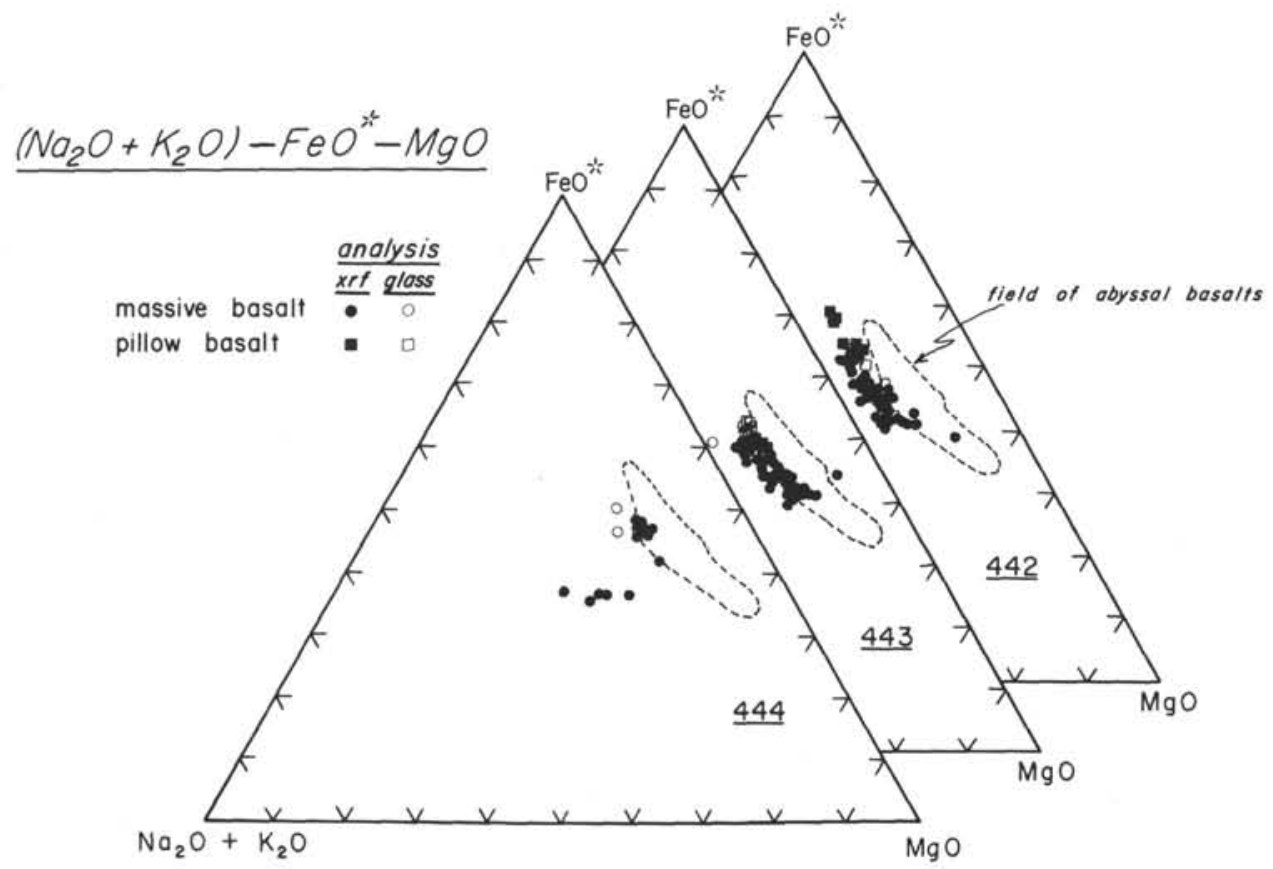

Figure 5. AFM diagram for basalts from the Shikoku Basin.

ther lithologic or geochemical boundaries and the uniformity of magnetic inclination between those boundaries suggest episodic volcanic activity with eruptive cycles of short duration and relatively long intervening hiatuses. It appears that the composition of the magma changed randomly during these hiatuses because of some combination of fractional crystallization, magma mixing, and tapping of different magmas representing primary variations in the source region.

\section{SUMMARY AND CONCLUSIONS}

The uppermost basalts at all three Shikoku Basin sites are sill complexes, including an alkali-basalt sill at Site 444. A similar, but larger sill complex with moreextensive alkaline rocks and far more intercalated sediment was also drilled at Site 446 in the Daito Basin to the southwest (Dick, in prep.). Sediment was found intercalated with the Site 444 sills and at the base of the Site 443 sill complex above the pillow lavas. At Sites 442 and 443 , the base of the sill complexes was completely penetrated, and the holes bottomed in extrusive complexes containing variable amounts of pillow lavas, sheet flows, and shallow sills. The basalts include tholeiites and olivine tholeiites whose average composition is close to the average of Melson et al. (1976) for midocean-ridge basalt glasses.

Geochemically, the pillow lavas and sills are distinct. In the $\mathrm{Al}_{2} \mathrm{O}_{3}-\mathrm{FeO}-\mathrm{MgO}$ discriminant diagram, the pillow lavas plot largely outside the abyssal-basalt field, while the sills plot within it. The pillow lavas are also more differentiated than the overlying sills; therefore, the two cannot be directly related. This does not preclude derivation of the pillow lavas by fractional crystal- lization from a melt similar in composition to the sill basalts at an earlier time.

The Shikoku Basin basalts, like most abyssal suites, plot along a single "pseudo-cotectic" trend in the normative olivine-plagioclase-pyroxene ternary which resembles the low-pressure experimental cotectic. This indicates that crystallization of these basalts was closely controlled by the phase boundaries; therefore, none represents an unmodified primary liquid from the mantle. As with the Cayman Trough and some Mid-Atlantic Ridge basalt suites, the position of the trend is offset from that of most MORB into the plagioclase-basalt field, indicating primary differences either in the composition of the primary source region, depth of segregation, or conditions of melting in the mantle.

Petrographically, the Shikoku basin basalts are typical of mid-ocean-ridge basalts. Principal phenocryst phases, in order of importance, are plagioclase, olivine, and clinopyroxene, olivine crystallizing earliest. Many of the basalts are aphyric ( $<1 \%$ phenocrysts), although phyric varieties are nearly as important as aphyric varieties at Site 443. Mineralogical variations are typical of abyssal tholeiites, except for the strongly alkaline trend of the pyroxene of the teschenite sill, and the potassium enrichment of plagioclase in the tholeiitic sill at Site 444. Except for the prominence of diabasic textures and features due to high vesicularity, the Shikoku Basin basalts are also texturally typical of MORB drilled elsewhere.

It is evident from the abrupt changes in composition and magnetic inclination that volcanism was episodic, with relatively long intervening hiatuses, during which the earth's poles wandered or reversed. It is unlikely that the abrupt changes in magnetic stratigraphy are due 


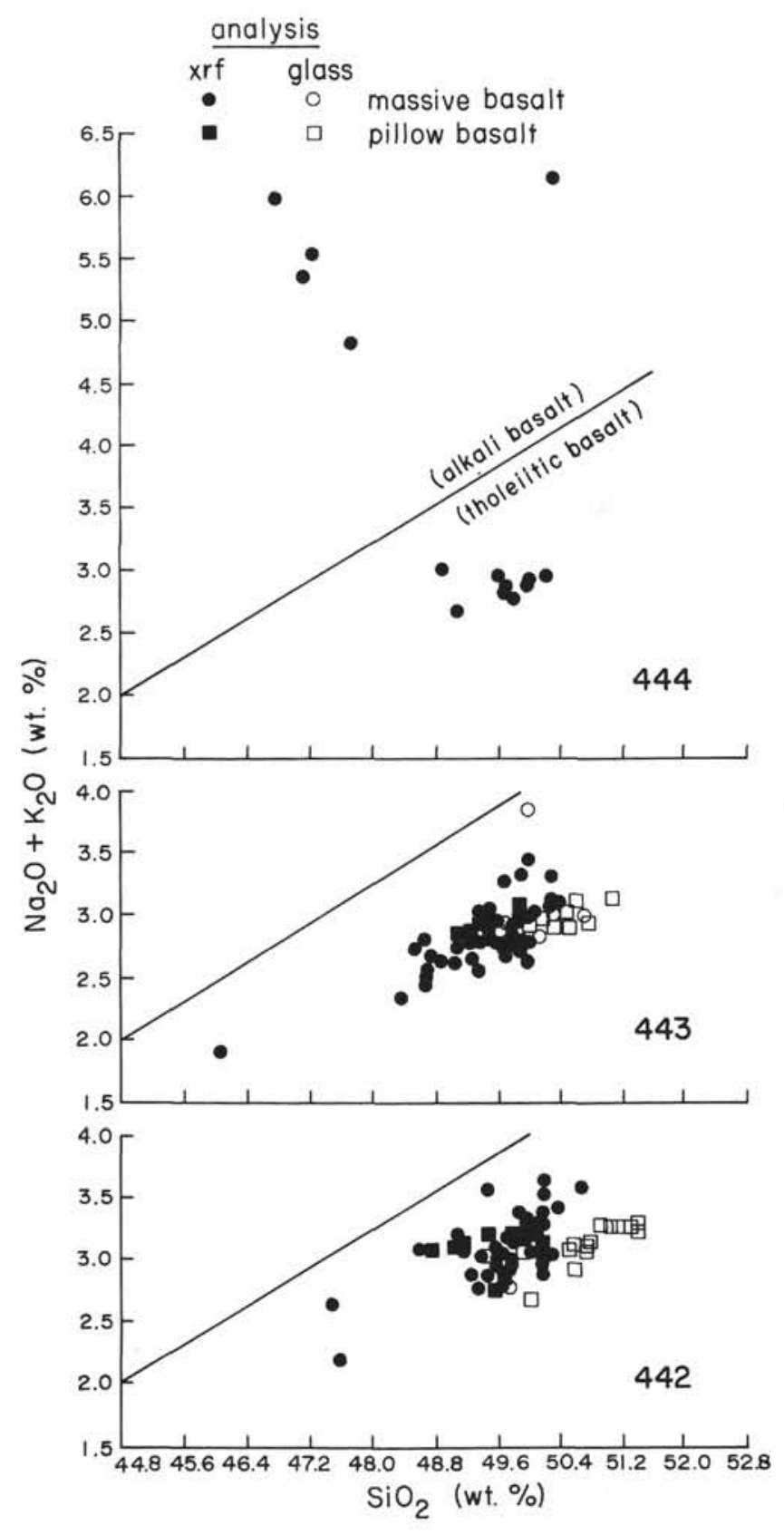

Figure 6. Alkalis versus silica. Line dividing alkaline and sub-alkaline fields is from Irvine and Baragar (1971).

solely to tectonic juxtaposition and rotation of blocks, because magnetic boundaries coincide with lithologic or geochemical boundaries in every case. There is also little evidence for tectonic disruption in the core material itself.

Because a reversal was found in the basement at both Sites 442 and 443 , it would be reasonable to guess that these short basement sections represent volcanism over a time at least on the order of the average period between reversals of the earth's magnetic field $(\sim 370,000$ years for this period; see Blakely, 1974). A longer period (0.7-2.0 m.y. minimum), however, is indicated by the pyrolized carbon at Site 444 (see Waples, this volume). In addition, the age of the oldest sediment intruded at Site 442 is 18 to 21 m.y., while that at the top of the first sill is 15 to 17 m.y., indicating at least a 1-m.y. period of volcanic activity (post-spreading center). Thus, given any reasonable spreading rate, the Shikoku Basin sites must represent extensive off-ridge volcanism across the basin.

The initial composition of each lava erupted in each series of sills and flows within an individual geochemical unit (or eruptive cycle), as indicated by the composition of the chill zones, varied little. Large differences, however, occur between different geochemical units. Thus, a number of processes must have occurred in a nearrandom manner during eruptive hiatuses, such as fractional crystallization and magma mixing in a magma chamber, or an entirely new magma may have been tapped.

At Site 442, there is little mineralogical evidence for significant involvement of a magma chamber, although variations in $\mathrm{Ni}$ and $\mathrm{Cr}$ suggest extensive crystal fractionation (Marsh et al., this volume). At Site 443 abundant phenocrysts in many lavas and some normal and reverse zoning of phenocrysts in the same flow do indicate the existence of a shallow magma chamber. It is likely, given the probable duration of the volcanism and therefore its infrequency at any one site, that such magma chambers were transitory.

It is unlikely that the alkaline and tholeiitic magmas at Site 444 are related, as indicated by their very different $\mathrm{TiO}_{2}$ contents at a given value of $\mathrm{FeO} / \mathrm{MgO}$ (Figure 9). Such different magmas might arise at the same site from early separation of low-percentage melts derived from magmaphile-element-rich vein materials in the mantle source region (alkaline basalts) and segregation of higher-percentage melts at more advanced degrees of mantle melting shallower in the mantle (tholeiites). Similar models are currently favored by a number of investigators to explain such bimodal volcanism (Tarney, pers. comm.; Hanson, pers. comm.). Alternatively the alkaline rocks may represent late off-axis activity above areas of relatively little mantle upwelling and therefore small degrees of melting.

Despite their close similarity to normal abyssal basalts, the Shikoku Basin tholeiites, other than the ubiquitous sills, are atypical of mid-ocean-ridge basalts in a number of features. Among these are the low apparent values of $\mathrm{Fe}-\mathrm{Mg} K_{\mathrm{D}}$ for olivine-glass pairs, indicating large initial $\mathrm{Fe}^{3+}$ contents; the consistently low analytical totals; and the very high vesicularities, which all indicate unusually high primary volatile contents, particularly $\mathrm{H}_{2} \mathrm{O}$ (see Dick, this volume).

The logical source of these volatiles is the slab in the subduction zone underlying the basin. The trace-element data also indicate a somewhat transitional character for the Shikoku Basin basalts, between MORB and both alkaline and arc-tholeiite suites. In addition, Pearce et al. (1977) noted that basalts such as the Triassic and Jurassic flood basalts, which appear to have erupted at the time of active breaking-up of continents, plot as a transitional suite across the boundary between 
- Pillows

Compound flow

Sheet flow

Sill
- Site 442

口 Site 443

- Site 444

Glass (microprobe analyses)

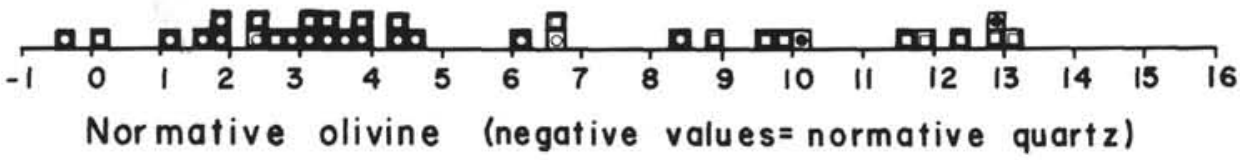

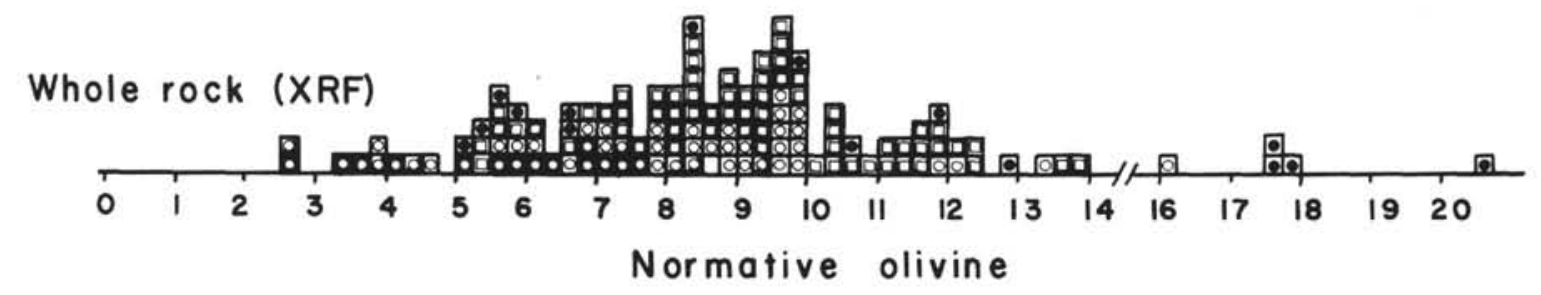

Figure 7. Histograms of normative-olivine content for Shikoku Basin basalts.

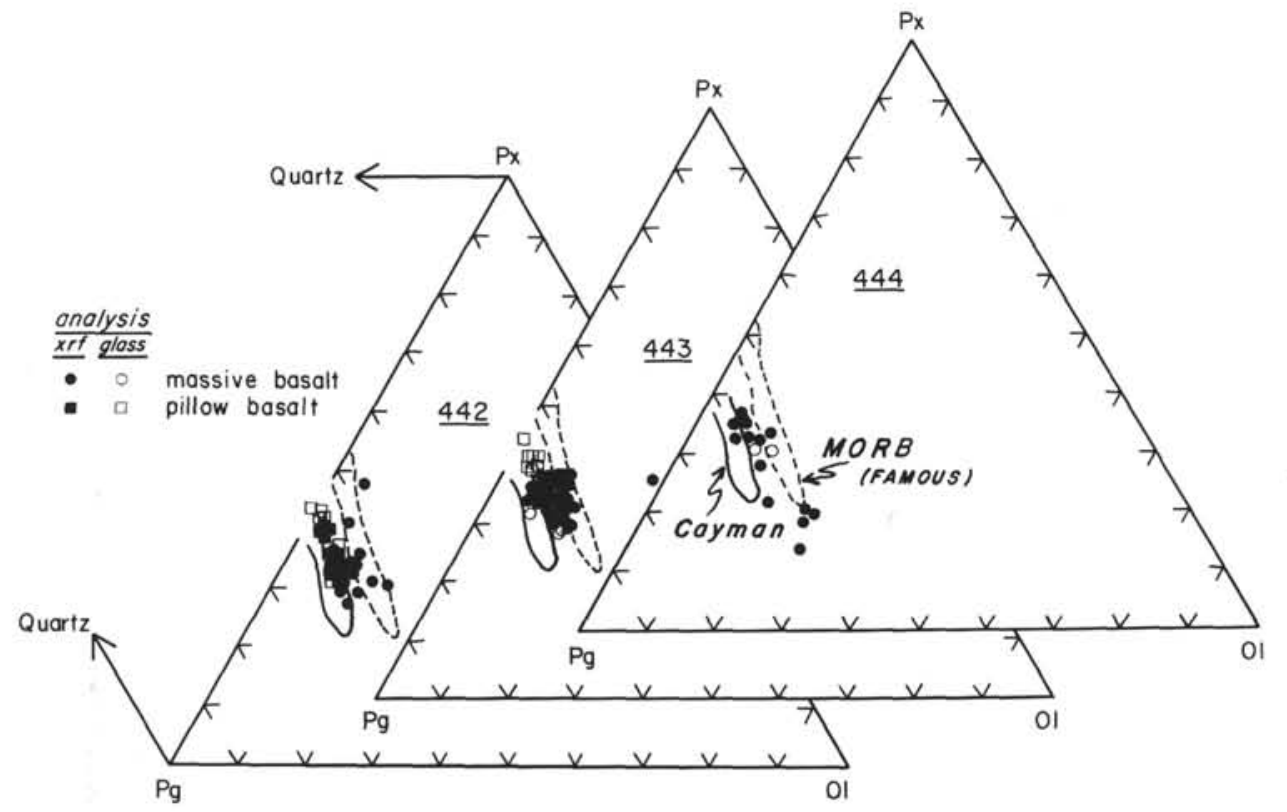

Figure 8. Normative plagioclase-pyroxene-olivine ternary fields for MORB and Cayman Trough basalts.

continental and abyssal basalts (Figure 10) and appear to have a somewhat oceanic character. Thus, Figure 10 suggests a similarity between the Shikoku Basin marginal-basin basalts and these Triassic and Jurassic basalts.

Much of what we report here has been previously documented in a number of other marginal basins. Dredge collections and a few drill cores have revealed that marginal-basin basalts are similar to abyssal tholeiites and include nearly the entire range of compositions reported from mid-ocean ridges (e.g., Hart et al., 1972; Sclater et al., 1972; Hawkins, 1977; Tarney et al., 1977).
Marginal-basin basalts with a transitional character have also been reported from the Lau Basin (Gill, 1976), some tending to be enriched in large-ion lithophile elements and having higher $\mathrm{Sr}^{87} / \mathrm{Sr}^{86}$ ratios than typical abyssal tholeiites (e.g., Saunders and Tarney, 1979; Hart et al., 1972). These basalts may be transitional between ocean-floor and island-arc tholeiites (Gill, 1976; Weaver et al., 1979). High water contents also have been reported before (e.g., Delaney et al., 1978; Garcia et al., 1979). It is evident then that the Shikoku Basin basalts, sharing these features, are typical of back-arc- 

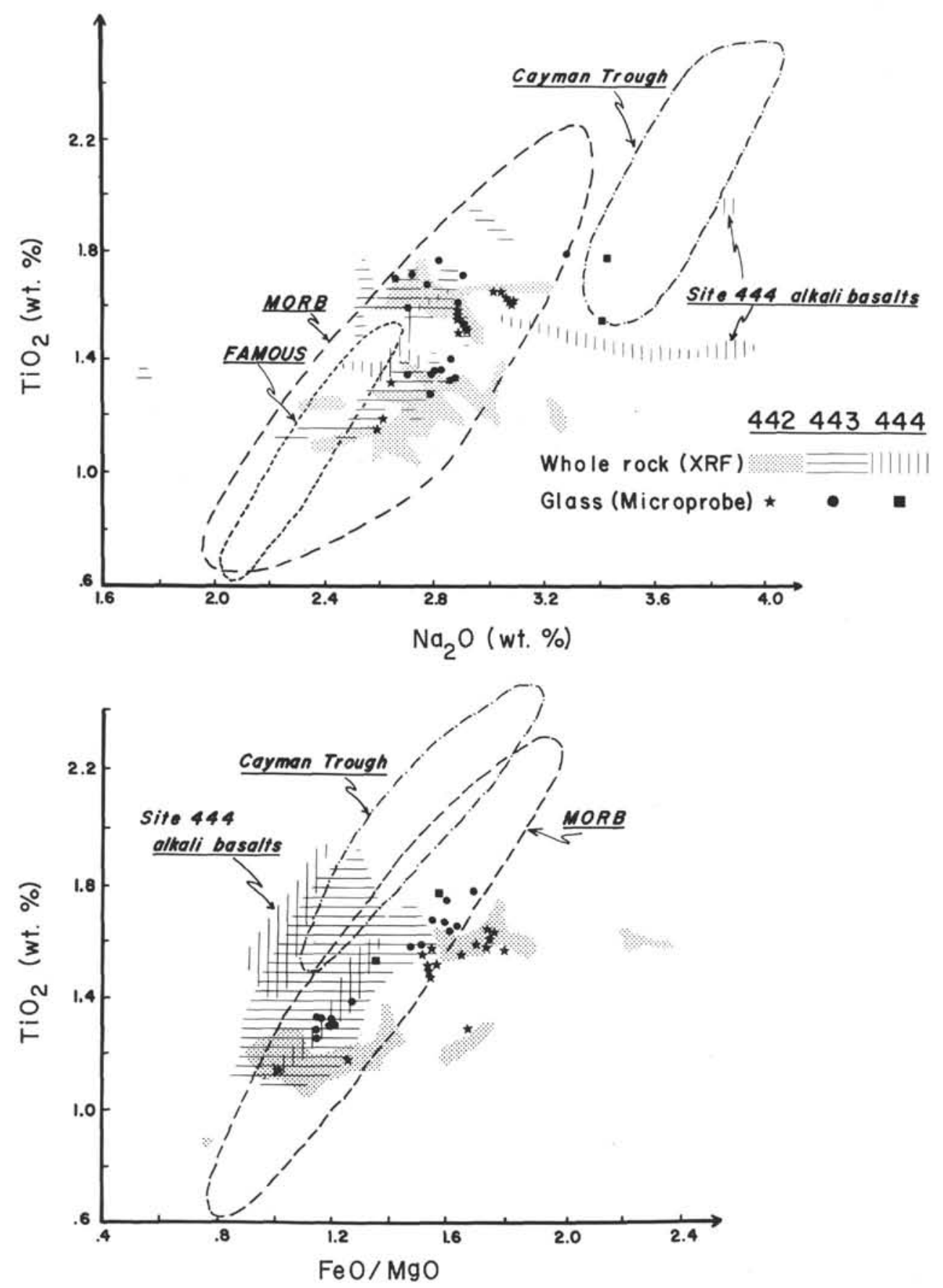

Figure 9. $\mathrm{TiO}_{2}$ versus $\mathrm{Na}_{2} \mathrm{O}$ and $\mathrm{FeO} / \mathrm{MgO}$ for Shikoku Basin basalts, with fields for MORB, FAMOUS, and Cayman Trough basalts from Thompson et al., (in press).

basin basalts. Perhaps the major new insight provided by Leg 58 is the realization that extensive sill and massive flows are also characteristic of back-arc basins.

Stratigraphically, the large number of sills is uncharacteristic of any of the DSDP holes in the Atlantic. However, similar sill complexes have been drilled in other back-arc basins, notably the Caribbean (Donnelly et al., 1973) and the South Fiji Basin (Stoeser, 1975). Although there were no deep basement penetrations in the Caribbean ( 25 m maximum), 13 massive basalt sills and flows were drilled at five widely spaced sites. As in the Shikoku and Daito Basins, alkaline as well as tholeiitic sills were prominent. It should be noted, however, that such sill complexes are also found in areas of mid-plate volcanism in the major ocean basins, as recently demonstrated by drilling in the Nauru Basin of the western Pacific (Scientific Party, Leg 61, 1978). Nonetheless, it appears that widespread off-ridge extensional volcanism is a characteristic feature of back-arc basins, as suggested by Lawver and Hawkins (1978) on geophysical 


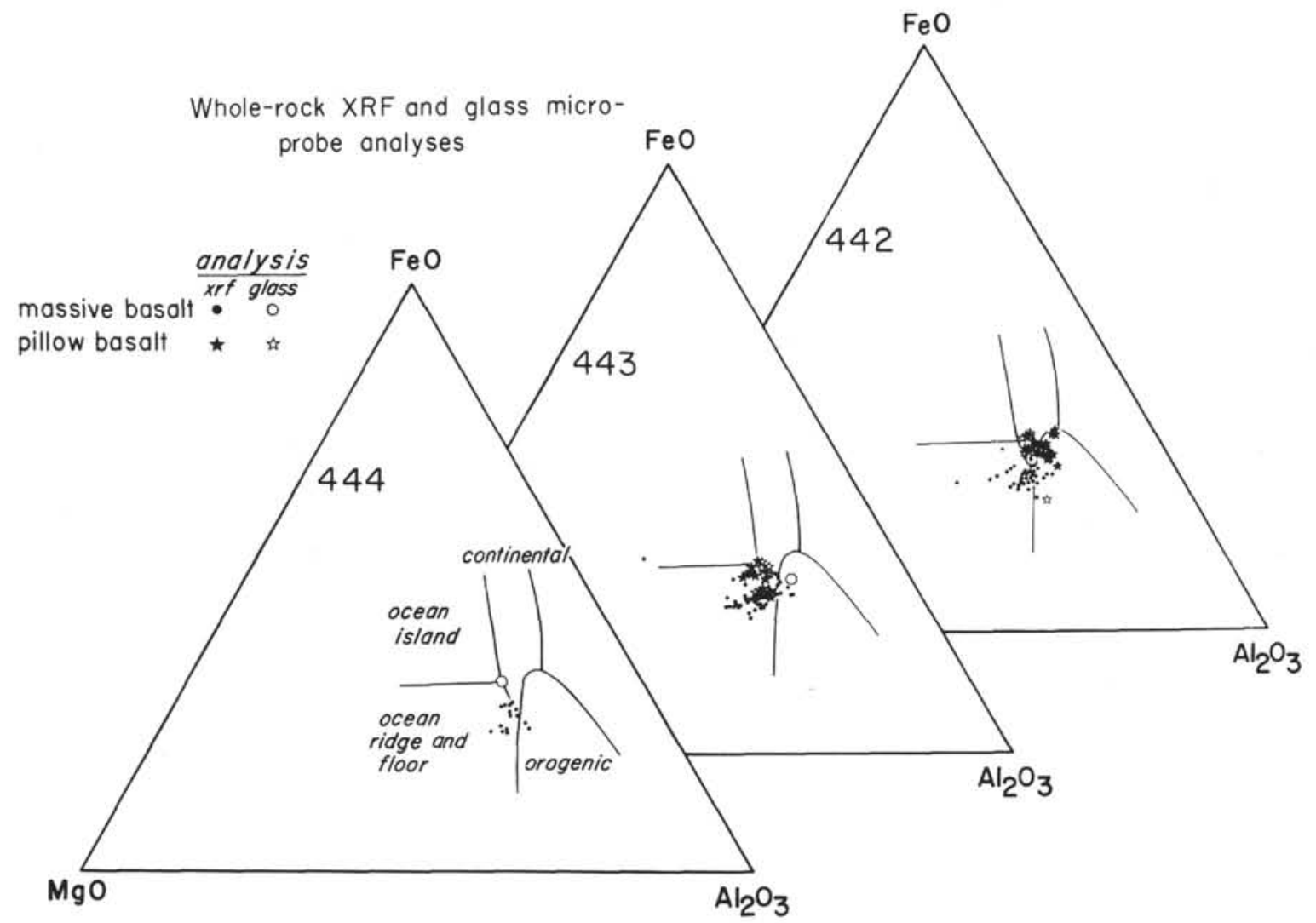

Figure 10. $\mathrm{MgO}-\mathrm{FeO}-\mathrm{Al}_{2} \mathrm{O}_{3}$ diagram, where $\mathrm{FeO}$ represents total iron. Discriminant boundaries for different tectonic environments for rocks with 51 to 56 per cent $\mathrm{SiO}_{2}$ from Pearce et al. (1977). Plotted on this diagram are alkaline as well as sub-alkaline basalts from Hole 444B. Basalts have not been screened for silica as the diagram remains effective as a discriminant for abyssal tholeiites with slightly lower silica contents (Pearce, pers. comm.).

grounds. The presence of alkaline volcanic rocks as well as tholeiitic rocks in the Daito, Shikoku and Caribbean basins is also consistent with their suggestion of an unusual prominence of "point source" volcanism (seamounts) in such regions.

It is clear from the poorly correlated linear magnetic anomalies in many marginal basins, including the Shikoku Basin (e.g., Kobayashi and Isezaki, 1976; Watts and Weissel, 1975), that spreading centers are present in back-arc basins. The mechanics of spreading, however, must differ significantly from mid-ocean ridges (see also Lawver and Hawkins, 1978), extension and volcanism occurring throughout the basin rather than just at a spreading center (ridge). The reasons for this are not entirely clear, but we agree with Lawver and Hawkins that it is the likely consequence of secondary mantle convection, due both to an induced counterflow of the old abyssal mantle above a descending slab, and to hydrous remelting of the overlying mantle (see Dick, this volume).

\section{IDENTIFICATION OF BACK-ARC-BASIN OPHIOLITES}

Back-arc basins are a favored provenance for many ophiolites, both for their small size and strategic position, and for their high heat flow, which should greatly facilitate the formation of crystalline nappes (Armstrong and Dick, 1974). Many ophiolites are now considered to have originated in such a manner (e.g., Upadhyay and Neale, 1979; Williams, 1975; Bakor et al., 1976). For example, the Josephine Peridotite in the western U. S. is a crystalline nappe emplaced along an eastward-dipping thrust over a contemporaneous calcalkaline volcanic pile (Rogue Formation). It far more likely came from a small collapsed marginal basin than from a major ocean to the west (Dick, 1977). However, structural grounds alone are not always definitive, and detailed stratigraphic information is required to place an ophiolite in its correct provenance. The details of stratigraphy and chemistry of the Shikoku basin sites provide a number of criteria to facilitate this placement:

1. The presence of interlayered sills, sediments and silicic ash. Such deposits immediately overlying or intercalcated with the ophiolite assemblage, as in the Mings Bight Ophiolite (Kidd et al., 1978), are probably the best criteria. The sill complexes, which are also found at Mings Bight and other Newfoundland ophiolites are, in view of the Nauru Basin results, only permissive evidence of a back-arc-basin origin. The presence of both alkalic and tholeiitic rocks may also be an important indicator (Lawver and Hawkins, 1978); their presence at the Daito, Shikoku, and Caribbean sites suggests that 
- whole-rock compositions by XRF analysis

- glass compositions by electron-microprobe analysis

Site 442 (oxides in wt.\%; dashed lines indicate geochemical breaks different from lithologic unit boundaries)

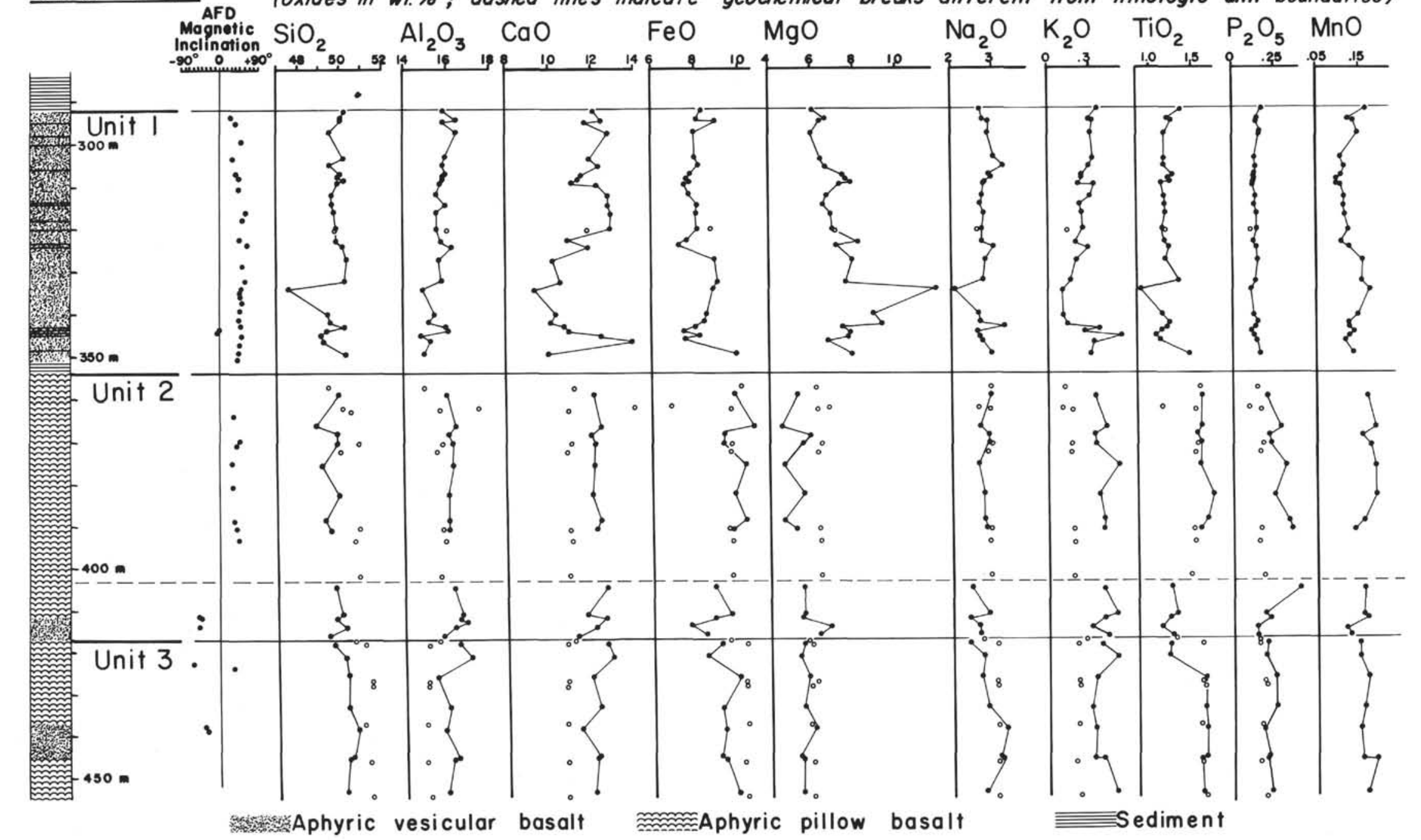

$\begin{array}{llllll}\mathrm{Na}_{2} \mathrm{O} & \mathrm{K}_{2} \mathrm{O} & \mathrm{TiO}_{2} & \mathrm{P}_{2} \mathrm{O}_{5} & \mathrm{MnO}\end{array}$

Figure 11. Whole-rock and glass compositions, Shikoko Basin sites. 


\section{Site 443}

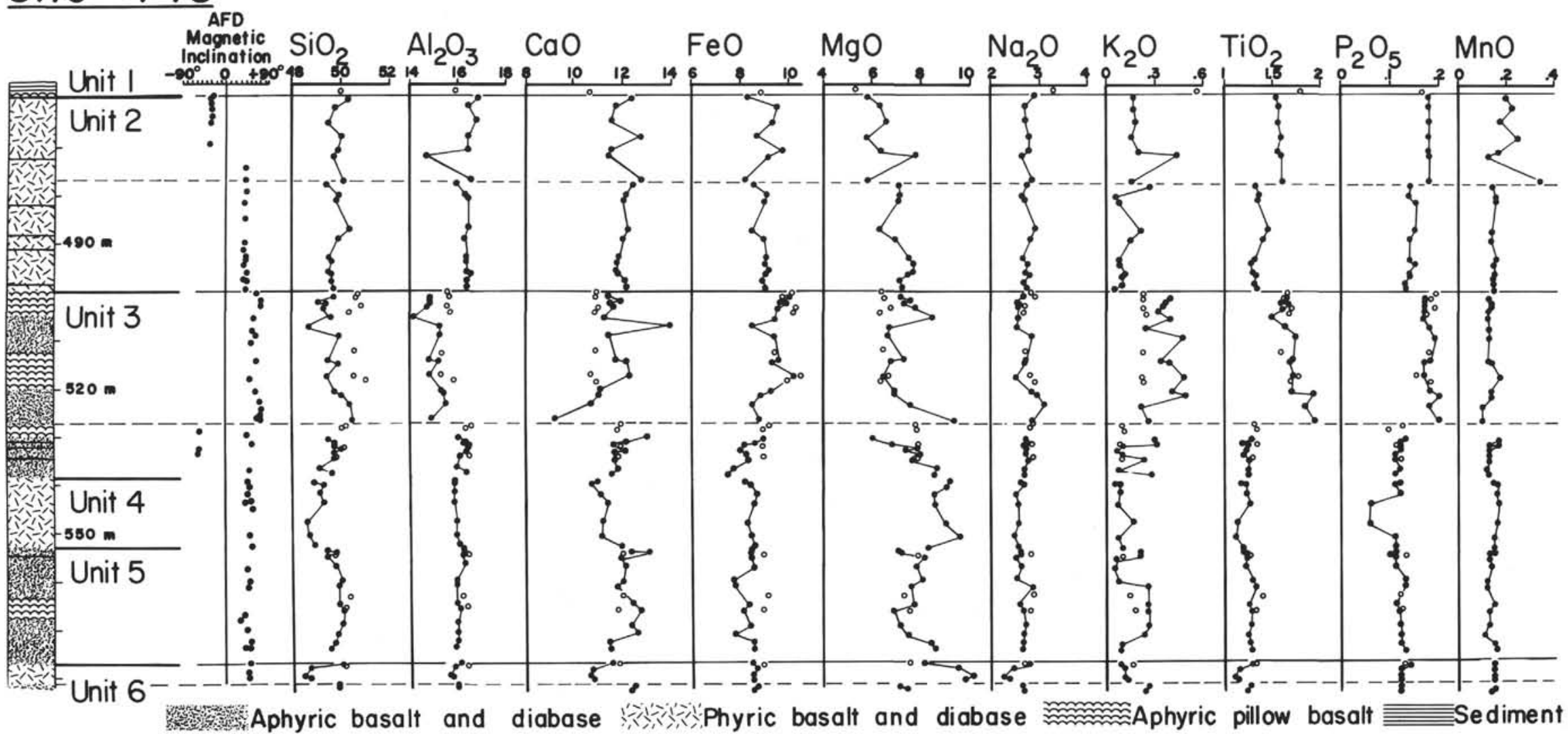

Figure 11. (Continued). 


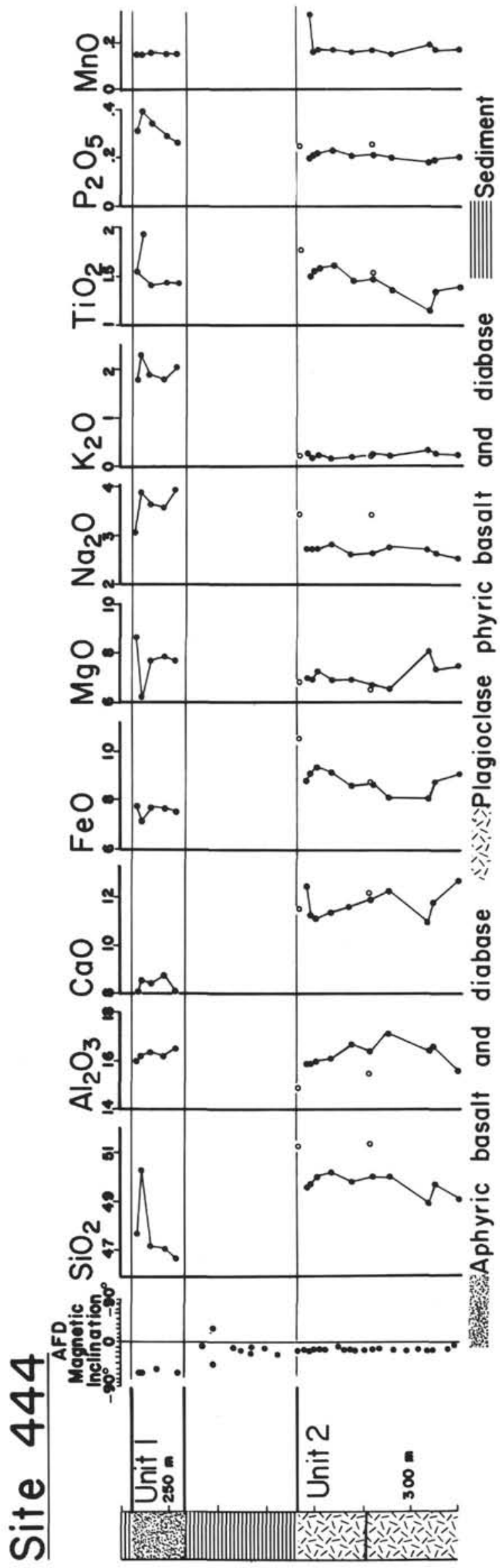

they are present in back-arc basins in greater abundance than in crustal sections derived from major ocean basins. In this light, we note that alkaline intrusives have been associated with Newfoundland ophiolites (e.g., Strong, 1974; Jamieson, 1977). Detailed study of the sediments themselves, indicating the proportions of terrigenous and pelagic components and their type, can yield substantial information on the type of basin, the depth of the eruptive site, and its proximity to land.

2 . High primary volatile contents (particularly water). It is evident that this is a characteristic feature of many (but not all) back-arc-basin basalts, but it is very unusual in typical MORB. While most ophiolites retain little fresh glass from which volatile contents can be measured directly, high vesicularity-particularly in association with deep abyssal sediments-may be a good indicator of a back-arc origin. A large contrast in vesicularity between rims and interiors of pillows may also indicate abundant water-rich volatiles (see Dick, this volume).

3. Transitional character of basalt. As previously noted, some basalts from back-arc basins are transitional between MORB and island-arc basalts (e.g. Gill, 1976; Weaver et al., 1979). While all discriminant diagrams should be used with caution, we note that Jabal Al Wask (Bakor et al., 1976) and the Bay of Islands complex (for both of which back-arc basin origins have been postulated) plot on the $\mathrm{Al}_{2} \mathrm{O}_{3}-\mathrm{Fe}-\mathrm{MgO}$ discriminant diagram in a manner similar to those from the Daito and Shikoku Basins (Figure 12).

\section{ACKNOWLEDGMENTS}

We thank the captain and crew of the Glomar Challenger. In addition, conversations with W. G. Melson, F. Chayes, J. Dewey, W. B. Bryan, S. R. Hart, M. Mottl, and G. Thompson have greatly aided our interpretation of the results. Microprobe analyses of basalt glasses were provided by W. G. Melson of the Smithsonian Institution and are gratefully acknowledged. This manuscript was written in large part during an extended visit at the University of Capetown, where the Departments of Geochemistry and Geology provided many helpful services. Funding was provided by grant \#OCE7726842 from the National Science Foundation. This is Woods Hole Oceanographic Institution Contribution No. 4406.

\section{REFERENCES}

Armstrong, R. L., and Dick, H. J. B., 1974. A model for the development of thin overthrust sheets of crystalline rock. Geology, 2, 35-40.

Bakor, A. R., Gass, I. G., and Neary, C. R., 1976. Jabal Al Wask, Northeast Saudi Arabia: An Eocambrian back-arc ophiolite. Earth Planet. Sci. Lett., 30, 1-9.

Bender, J. F., Hodges, F. N., and Bence, A. E., 1978. Petrogenesis of basalts from the project FAMOUS area: experimental study from 0 to $15 \mathrm{~kb}$. Earth Planet. Sci. Lett., 41, 277-302.

Blakely, R. J., 1974. Geomagnetic reversals and crustal spreading rates during the Miocene. J. Geophys. Res., 79, 2974-2985.

Deer, W. A., Howie, R. A., and Zussman, J., 1963. Rock Forming Minerals (Vol. 4) Framework Silicates: New York (John Wiley and Sons).

Delaney, J. R., Muenow, D. W., and Graham, D. G., 1978. Abundance and distribution of water, carbon, and sulphur 


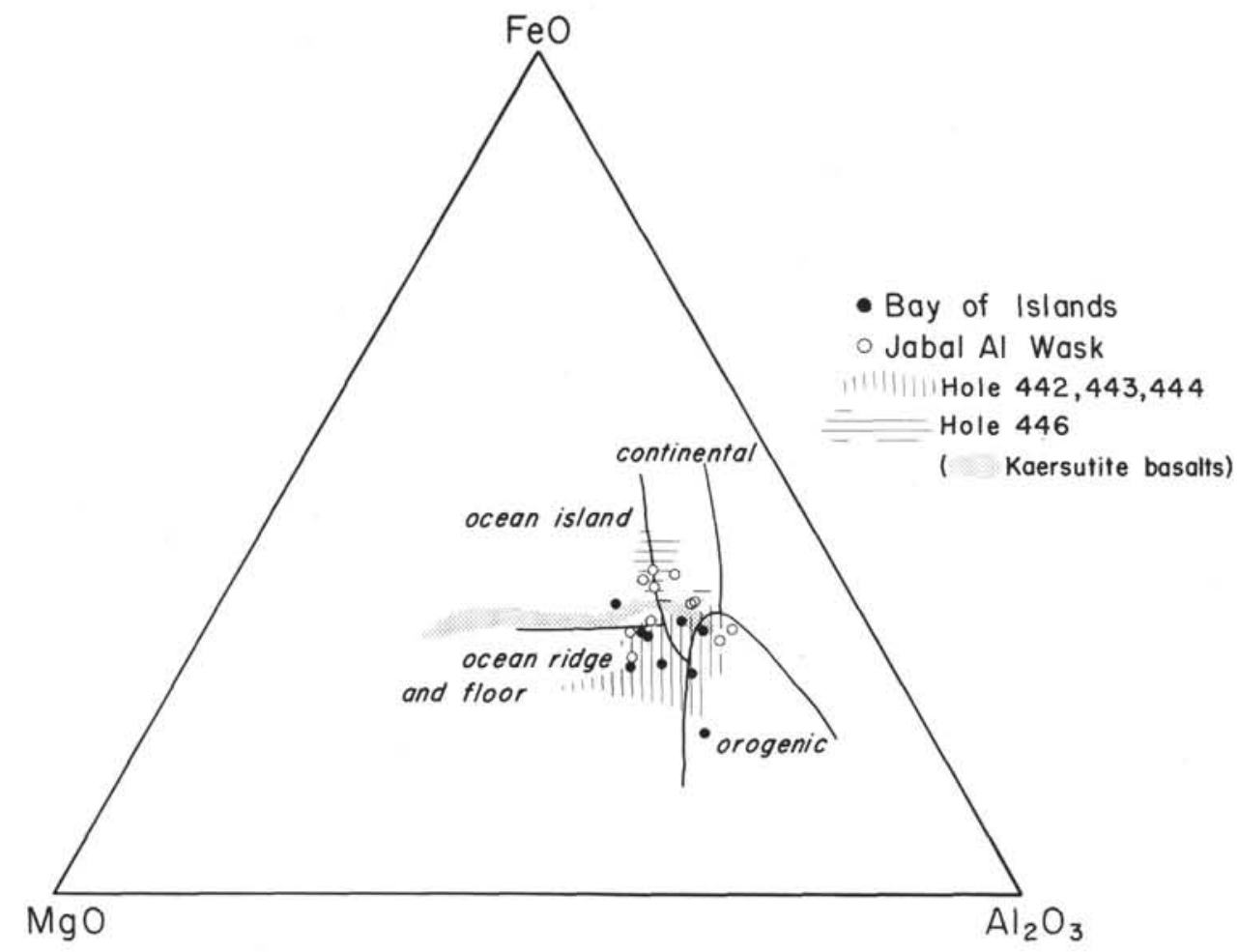

Figure 12. $\mathrm{MgO}-\mathrm{FeO}-\mathrm{Al}_{2} \mathrm{O}_{3}$ discriminant diagram for Bay of Islands complex (Williams and Malpas, 1972) and Jabal Al Wask (Bakor et al., 1976) pillow lavas and dikes. Field of analyses for Shikoku and Daito Basins are indicated by shading. Field for the Shikoku Basin from Dick et al., (this volume).

in the glassy rims of submarine pillow basalts. Geochim. Cosmochim. Acta, 42, 581-594.

Dick, H. J. B., 1977. Partial melting in the Josephine Peridotite. I. The effect of mineral composition and its consequence for geobarometry and geothermometry. Am. J. Sci., 277, 801-832.

, in prep. Petrology of the Minami Daito Basin.

Dick, H. J. B., and Bryan, W. B., 1978. Variation of basalt phenocryst, mineralogy and rock compositions in DSDP Hole 396B. In Dmitriev, L., Heirtzler, J., et al., Init. Repts. DSDP, 46: Washington (U.S. Govt. Printing Office), pp. 215-225.

Donnelly, T. W., Melson, W., Kay, R., and Rogers, J. J. W., 1973. Basalts and dolerites of late Cretaceous age from the Central Caribbean. In Edgar, N. T., Saunders, J. B., et al., Init. Repts. DSDP, 15: Washington (U.S. Govt. Printing Office) pp. 989-1012.

Dungan, M. A., Rhodes, J. M., Long, P. E., Blanchard, D. P., Brannon, J. C., and Rodgers, K. V., 1978. The petrology and geochemistry of basalts from Site 396, Legs 45 and 46 of the Deep Sea Drilling Project. In Dmitriev, L., Heirtzler, J. et al., Init. Repts. DSDP, 46: Washington (U.S. Govt. Printing Office) pp. 89-114.

Garcia, M. O., Liu, N. W. K., and Muenow, D. W., 1979. Volatiles in submarine volcanic rocks from the Mariana island arc and trough. Geochim. Cosmochim. Acta, 43, 305-312.

Gill, J. B., 1976, Composition and age of Lau Basin and Ridge volcanic rocks: implications for the evolution of an interarc basin and remanent arc. Geol. Soc. Am. Bull., 87, 1384-1395.
Hart, S. R., Glassley, W. E., and Karig, D. E., 1972. Basalts and seafloor spreading behind the Mariana Island arc. Earth Planet. Sci. Lett., 15, 12-18.

Hawkins, J. W., 1977. Petrologic and geochemical characteristics of marginal basin basalts. In Talwani, M., and Pitman, W. C. (Eds.), Island Arcs, Deep Sea Trenches and BackArc Basins: Washington (Am. Geophys. Union), pp. 355365.

Hodges, F. N., and Papika, J. J., 1977. Petrology of basalts, gabbros, and peridotites from DSDP Leg 37. In Aumento, F., Melson, W. G., et al., Init. Repts. DSDP, 37: Washington, (U.S. Govt. Printing Office) pp. 711-719.

Irvine, T. N., and Baragar, W. R. A., 1971. A guide to the chemical classification of the common volcanic rocks. Can. J. Earth Sci., 8, 523-548.

Jamieson, R. A., 1977. A suite of alkali basalts and gabbros associated with the Hare Bay Allochthon of western Newfoundland. Can. J. Earth Sci., 14, 346-356.

Karig, D. W., 1970. Ridges and basins of the Tonga-Kermadec island arc system. J. Geophys. Res., 75, 239-255.

Kennedy, G. C., 1948. Equilibrium between volatiles and iron oxides in igneous rocks. Am. J. Sci., 246, 529-549.

Kidd, W. S., Dewey, J. F., and Bird, J. M., 1978. The Mings Bight Ophiolite Complex, Newfoundland: Appalachian oceanic crust and mantle. Can. J. Earth Sci., 15, 781-804.

Kobayashi, K., and Isezaki, 1976. Magnetic anomalies in the sea of Japan and the Shikoku Basin: possible tectonic implications. Am. Geophys. Union Monogr., 19.

Kuno, H., 1955. Ion substitution in the diopside-ferropigeonite series of clinopyroxenes, Am. Mineral., 40, 70-93. 
1968. Differentiation of basalt magmas. In Hess, H., and Poldervaart, A. (Eds.), Basalts (Vol. 2): New York (John Wiley and Sons,) pp. 623-688.

Kushiro, I., 1960. Si-Al relation in clinopyroxenes from igneous rocks. Am. J. Sci., 258, 548-554.

1964. Petrology of the Atumi dolerite, Japan. Univ. Tokyo J. Fac. Sci., Sec. II, 14 (2), 135-202.

Lawver, L. A., and Hawkins, J. W., 1978. Diffuse magnetic anomalies in marginal basins: their possible tectonic and petrologic significance. Tectonophysics, 43, 323-339.

LeBas, M. J., 1962. The role of aluminum in igneous clinopyroxenes with relation to their parentage. Am. J. Sci., 260, 267-288.

Melson, W. G., and Thompson, G., 1971. Petrology of a transform fault zone and adjacent ridge segments. Trans. Roy. Phil. Soc. London, Ser. A, 268, 423-442.

Melson, W. G., Vallier, T. L., Wright, T. L., Byerly, G., and Nelen, J., 1976. Chemical diversity of abyssal volcanic glass erupted along Pacific, Atlantic and Indian Ocean sea-floor spreading centers. In Geophysics of the Pacific Ocean Basin: Am. Geophys. Union Monogr., 19, 351-367.

Mevel, C., Ohnenstetter, D., and Ohnenstetter, M., 1978. Mineralogy and petrography of Leg 46 basalts. In Dmitriev, L., Heirtzler, J., et al., Init. Repts. DSDP, 46: Washington (U.S. Govt. Printing Office), pp. 151-164.

Miyashiro, A., 1978. Nature of alkalic volcanic rock series. Contr. Mineral. Petrol., 66, 91-104.

Miyashiro, A., Shido, F., and Ewing, M., 1970. Crystallization and differentiation in abyssal tholeiites and gabbros from mid-ocean ridges. Earth Planet. Sci. Lett., 7, 361-365.

Murauchi, S., Den, N., Asano, S., Hotta, H., Yoshii, T., Asanuma, K., Hagiwara, K., Ichikawa, K., Sato, T., Ludwig, W. J., Ewing, J., Edgar, N. T., and Houtz, R. E., 1968. Crustal structure in the Phillipine Sea. J. Geophys. Res., 73, 3143-3171.

Murauchi, S., and Asanuma, T., 1974. Seismic profiler and sonobuoy refraction measurements during GDP-6 to -8 voyages. Mar. Sci., 6, 23-27.

O'Hara, M. J., 1968. Are ocean floor basalts primary magmas? Nature, 220, 683-686.

Pearce, T. H., Gorman, B. E., and Birkett, T. C., 1977. The relationship between major element chemistry and tectonic environment of basic and intermediate volcanic rocks. Earth Planet. Sci. Lett., 36, 121-132.

Roeder, P. L., and Emslie, R. F., 1970. Olivine-liquid equilibrium. Contr. Mineral. Petrol., 29, 277-307.

Presnall, D. C., Dixon, S. A., Dixon, J. R., O'Donnell, T. H., Brenner, M. L., Schrock, R. L., and Dycus, D. W., 1978. Liquidus phase relations on the join diopside-forsterite-anorthite from $1 \mathrm{~atm}$ to $2 \mathrm{~kb}$ : their bearing on the generation and crystallization of basaltic magma. Contr. Mineral. Petrol., 66, 203-220.
Saunders, A., and Tarney, J., 1979. The geochemistry of basalts from a back-arc spreading centre in the East Scotia Sea. Geochim. Cosmochim. Acta, 43, 555-572.

Sclater, J. G., Hawkins, J. W., Mammerickx, J., Chase, C. G., 1972. Crustal extension between Tonga and Lau ridges: petrologic and geophysical evidence. Geol. Soc. Am. Bull., 83, 505-518.

Scientific Party, Leg 61, 1978. Volcanic complex found. Geotimes, 23, 21-24.

Stoeser, D. B., 1975. Igneous rocks from Leg 30 of the Deep Sea Drilling Project. In Andrews, J. E., Packham, G., et al., Init. Repts. DSDP, v. 30: Washington (U.S. Govt. Printing Office), pp. 401-414.

Strong, D. F., 1974. An off-axis volcanic suite associated with the Bay of Islands Ophiolites, Newfoundland. Earth Planet. Sci. Lett., 21, 301-309.

Tarney, J., Saunders, A. D., and Weaver, S. D., 1977. Geochemistry of volcanic rocks from the island-arcs and marginal basins of the Scotia Arc region. In Talwani, M., and Pitman, W. C. III (Eds.), Island Arcs, Deep Sea Trenches and Back Arc Basins: Washington (Am. Geophys. Union.), pp. 367-377.

Thompson, G., Bryan, W. B., and Melson, W. G., in press. Geological and geophysical investigation of the midCayman rise spreading center: geochemical variation and petrogenesis of basalt glasses. J. Geol.

Upadhyay, H. D., and Neale, E. R. W., 1979, On the tectonic regimes of ophiolite genesis. Earth Planet. Sci. Lett., , 43, 93-102.

Uyeda, S., 1976. Heat flow. In Crust and Upper Mantle of the Japanese Area, (Part 1): Tokyo (Jap. Comm. UMP), pp. 97-105.

Watts, A. B., and Weissel, J. K., 1975. Tectonic history of the Shikoku marginal basin. Earth Planet. Sci. Lett., 25, 239-250.

Weaver, S. D., Saunders, A. D., Pankhurst, R. J., and Tarney, J., 1979. A geochemical study of magmatism associated with the initial stages of back-arc spreading: the Quaternary volcanics of the Bransfield Strait, from South Shetland islands. Contr. Mineral. Petrol., 68, 151-169.

Wilkinson, J. F. G., 1958. The petrology of a differentiated teschenite sill near Gunedah, New South Wales. Geol. Mag., 94, 123-134.

1968. The petrography of basaltic rocks. In Hess, H., and Poldervaart, H. (Eds.), Basalts (Vol. 1): New York, (John Wiley and Sons), pp. 163-214.

Williams, H., 1975. Structural succession, nomenclature and interpretation of transported rocks in western Newfoundland. Can. J. Earth Sci., 12, 1874-1894.

Williams, H., and Malpas, J., 1972. Sheeted dikes and brecciated dike rocks within transported igneous complexes, Bay of Islands, Western Newfoundland. Can. J. Earth Sci., 9, 1216-1229. 
PLATE 1

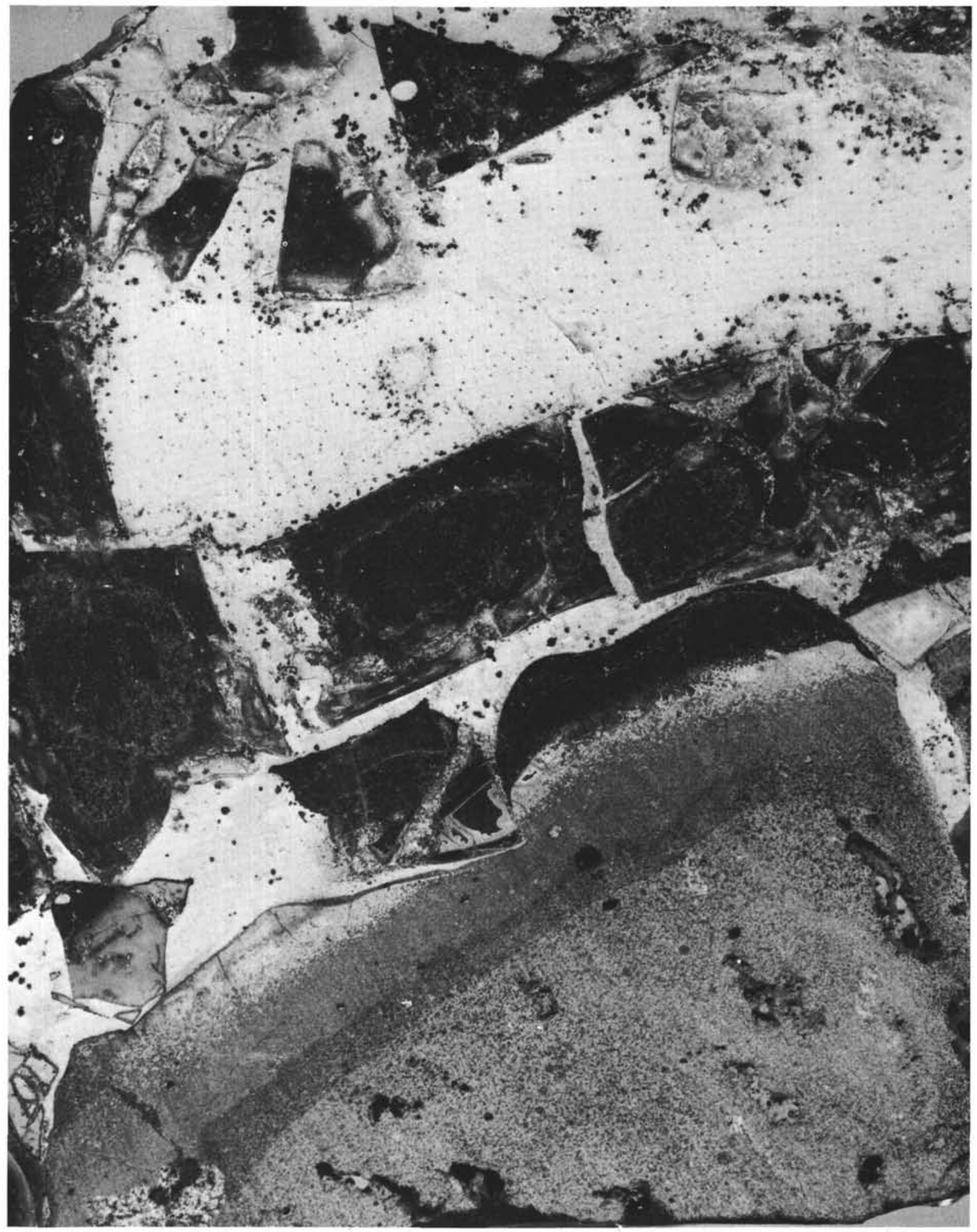

Nannofossil chalk and palagonite breccia drilled from the interstices between basalt pillows in Unit 1 at Hole 442B. Margin of one pillow shown at the left side of the photo. 
PLATE 2

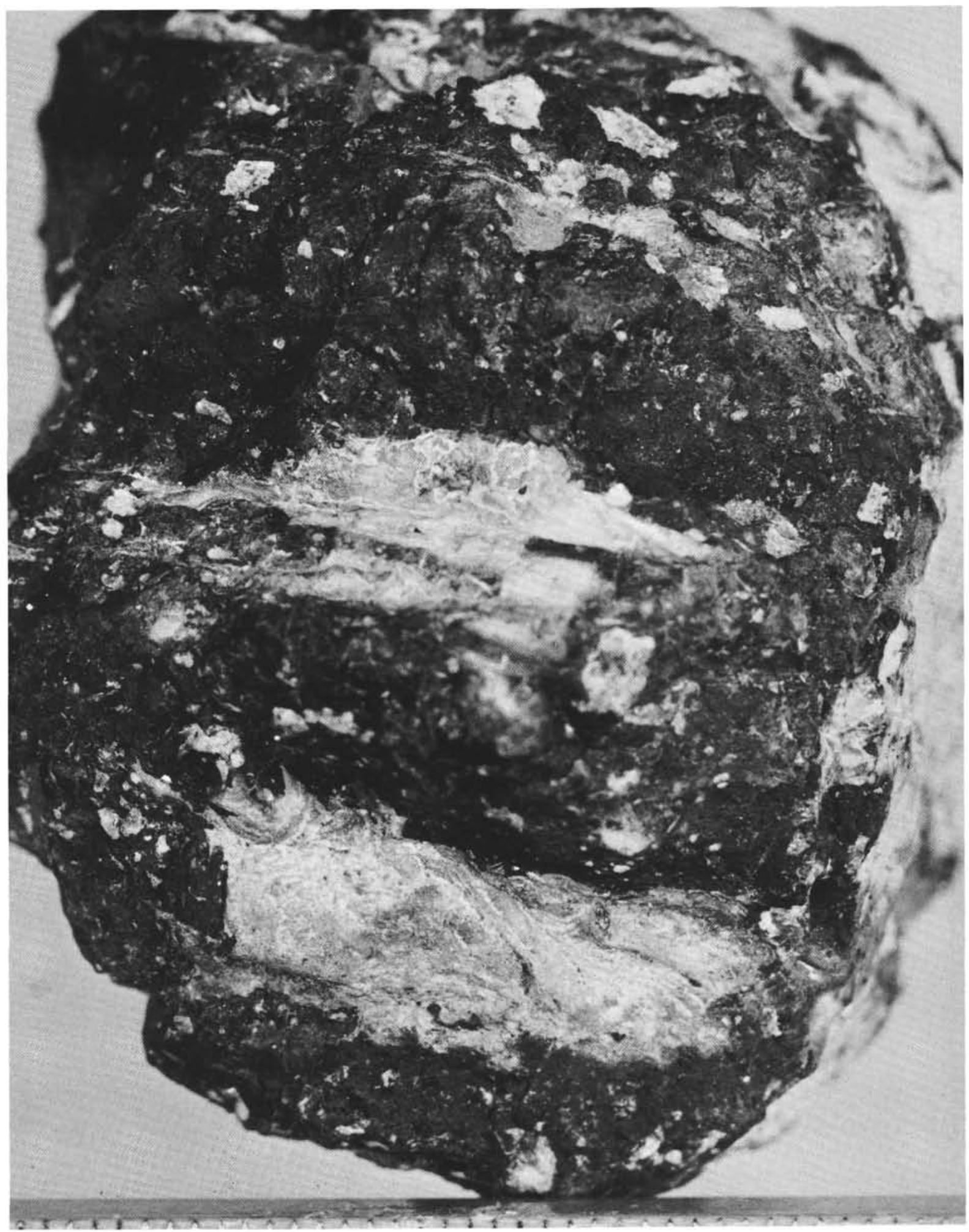

Ropey flow surface from the top of Unit 2 at Hole 444A. Whitish encrustation appears to be baked sediment. Although the unit appears to be intruded into the sediment and is therefore a sill, it could also be termed a sub-sediment flow. 